\title{
MOLECULAR AND THERMAL DIFFUSION COEFFICIENTS OF N-DODECANE, ISOBUTYLBENZENE AND TETRAHYDRONAPHTALENE HYDROCARBON MIXTURES IN POROUS MEDIA
}

by

Mustafa Khawaja, B. Eng.

Ryerson University, 2004

\author{
A thesis \\ presented to Ryerson University \\ in partial fulfillment of the \\ requirements for the degree of \\ Master of Applied Science \\ in the program of \\ Mechanical Engineering
}

Toronto, Ontario, Canada, 2005

(C) Mustafa Khawaja 2005 


\section{UMI Number: EC53031}

\section{All rights reserved \\ INFORMATION TO USERS}

The quality of this reproduction is dependent upon the quality of the copy submitted. Broken or indistinct print, colored or poor quality illustrations and photographs, print bleed-through, substandard margins, and improper alignment can adversely affect reproduction.

In the unlikely event that the author did not send a complete manuscript and there are missing pages, these will be noted. Also, if unauthorized copyright material had to be removed, a note will indicate the deletion.

\section{$\mathrm{UMI}^{\circ}$}

UMI Microform EC53031

Copyright 2008 by ProQuest LLC

All rights reserved. This microform edition is protected against unauthorized copying under Title 17, United States Code.

ProQuest LLC

789 East Eisenhower Parkway

P.O. Box 1346

Ann Arbor, MI 48106-1346 


\section{AUTHOR'S DECLARATION}

I hereby declare that I am the sole author of this thesis.

I authorize Ryerson University to lend this thesis to other institutions or individuals for the purpose of scholarly research.

I further authorize Ryerson University to reproduce this thesis by photocopying or by other means, in total or in part, at the request of other institutions or individuals for the purpose of scholarly research. 


\section{BORROWER'S PAGE}

Ryerson University requires the signatures of all persons using or photocopying this thesis. Please sign below and give the address and date. 


\title{
ABSTRACT \\ MOLECULAR AND THERMAL DIFFUSION COEFFICIENTS OF N-DODECANE, ISOBUTYLBENZENE AND TETRAHYDRONAPHTALENE HYDROCARBON MIXTURES IN POROUS MEDIA
}

\author{
Master of Applied Science 2005, Mustafa Khawaja \\ School of Graduate Studies, Mechanical Engineering, Ryerson University
}

The thermal diffusion phenomenon was studied in detail by utilizing mixtures consisting of normal alkenes and two aromatics (n-Dodecane, Isobutylbenzene, and 1,2,3,4Tetrahydronaphtalene) based on comparison with available experimental data. This study presents the first report of a comparison of thermal diffusion coefficients of a ternary hydrocarbon mixture with experimental data in the literature. In addition to thermal diffusion coefficients, molecular diffusion coefficients are also measured and compared with Benchmark experimental values for three binary mixtures. Furthermore, molecular and thermal diffusion coefficients for three binary mixtures are used to correlate and estimate the thermal diffusion coefficients in the ternary hydrocarbon mixture. The thermo-solutal convection in porous media was simulated numerically using the Firoozabadi model in order to investigate the composition variation due to the processes of thermal diffusion and convection. Finally, a multi-porosity/multi-permeability model was utilized to further analyze the processes of thermal diffusion and convection in fractured porous media. 


\section{ACKNOWLEDGEMENTS}

In the name of Allah, the most Compassionate, the most Merciful.

And what reason have we that we should not rely on Allah? And He has indeed guided us in our ways; and certainly we would bear with patience your persecution of us; and on Allah should the reliant rely. (The Holy Quran, 014.012) And He gives you of all that you ask Him; and if you count Allah's favours, you will not be able to number them; most surely man is very unjust, very ungrateful. (The Holy Quran, 014.034) My Lord! Make me keep up prayer and from my offspring (too), O our Lord, and accept my prayer: O our

Lord! Grant me protection and my parents and the believers on the day when the reckoning shall come to pass! (The Holy Quran, 014.040-0.41)

It has been a privilege and an honour to work with Dr. Ziad Saghir of Ryerson University and the author would like to thank Dr. Ziad Saghir for his helpful assistance and guidance throughout the completion of this thesis. The author also acknowledges the support of Dr. Yu Claire Yan, Dr. Charles Guobing Jiang, Dr. Mohammed Chacha, and Dr. Stephan Van Vaerenbergh for their assistance and useful suggestions.

The author also would like to express his sincere appreciation to both his parents Sajjad and Gul Khawaja for their complete support and enthusiasm in the completion of this thesis. The author also appreciates the support and affection of his siblings, Annie and Jemsheed Dean, Amber Khawaja, and Mujtaba Khawaja. The author also likes to express his appreciation and affection for his nephew Zakariya Dean.

The author also acknowledges the useful suggestions of his colleagues Omar Gaber, Jon Bahen, Shu Pan, Chandra Adimoolam, Mahwish Sohail, and Tawfiq Jaber.

The author also acknowledges the useful suggestions and encouragement of Litifa Samra Noor. 


\section{TABLE OF CONTENTS}

AUTHOR'S DECLARATION $\quad$ ii

BORROWER'S PAGE

ABSTRACT $\quad$ iv

ACKNOWLEDGEMENTS $\quad \mathbf{v}$

TABLE OF CONTENTS

LIST OF FIGURES $\quad$ ix

LIST OF TABLES $\quad \therefore \quad$ xi

NOMENCLATURE

CHAPTER 1: INTRODUCTION

1.1 LITERATURE REVIEW

1.2 RESEARCH OBJECTIVES $\quad$ I2

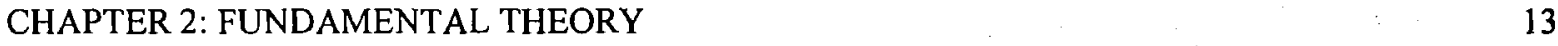

2.1 THEORETICAL MODELS OF THERMAL DIFFUSION

2.1.1 Haase Model 13

2.1.2 Kempers Model $\quad$ I5

2.1.3 Firoozabadi Model 15

2.2 FUNDAMENTAL EQUATIONS IN POROUS MEDIA 17

2.2.1 Momentum Equation $\quad 18$

2.2.2 Conservation of Mass 20

2.2.3 Energy Equation $\quad 22$

2.2.4 Boussinesq Approximation $\quad 24$

CHAPTER 3: THEORETICAL ANALYSIS AND COMPARISON WITH EXPERIMENTAL DATA FOR THE MOLECULAR AND THERMAL DIFFUSION COEFFICIENTS FOR A TERNARY MIXTURE 26

3.1 INTRODUCTION

3.2 THEORETICAL DERIVATION OF THE FIROOZABADI MODEL 
3.4 EQUATION OF STATE: PENG-ROBINSON (PR) 38

3.5 THERMAL AND MOLECULAR DIFFUSION: EXPERIMENTAL APPROACH 40

3.6 RESULTS AND DISCUSSION

\begin{tabular}{ll}
3.7 & SUMMARY \\
\hline
\end{tabular}

CHAPTER 4: TWO DIMENSIONAL SIMULATION OF THERMAL DIFFUSION IN POROUS MEDIA

$\begin{array}{ll}4.1 \text { CONVECTION IN POROUS MEDIA } & 49\end{array}$

$\begin{array}{ll}4.2 \\ \text { THE NUMERICAL MODEL } & 49\end{array}$

4.3 MATHEMATICAL MODELLING 51

4.3.1 Mass Continuity Equation 51

4.3.2 Momentum Conservation Equation 51

4.3.3 Energy Conservation Equation $\quad 52$

4.4 NUMERICAL SOLUTION TECHNIQUE $\quad 52$

4.5 MESH SENSITIVITY ANALYSIS $\quad 56$

4.6 RESULTS AND DISCUSSION $\quad 58$

4.6.1 Comparison of flow patterns $\quad 59$

4.6.2 Comparison of Density distribution along the center of the cavity vertically and horizontally

$\begin{array}{ll}\text { 4.6.3 Variation of Concentration with permeability } & 70\end{array}$

$\begin{array}{ll}\text { 4.6.4 Separation Ratio } & \mathbf{7 4}\end{array}$

$\begin{array}{ll}4.7 \text { SUMMARY } & 79\end{array}$

CHAPTER 5: TWO DIMENSIONAL SIMULATION OF THERMAL DIFFUSION IN A HETEROGENEOUS POROUS MEIDA $\quad \mathbf{8 1}$

$\begin{array}{ll}5.1 \text { INTRODUCTION } & \mathbf{8 1}\end{array}$

5.2 THE NUMERICAL MODEL AND VALIDATION $\quad 82$

$\begin{array}{ll}5.3 \text { RESULTS AND DISCUSSION } & 84\end{array}$

$\begin{array}{ll}\text { 5.3.1 Flow patterns } & 84\end{array}$

5.3.2 Comparison of concentration inside the cavity $\quad 89$

5.3.3 SORET COEFFICIENT COMPARISON FOR HORIZONTAL AND VERTICAL

FRACTURE CASES $\quad 91$ 


\subsection{SUMMARY}

CHAPTER 6: CONCLUSION

REFERENCES

APPENDIX A

APPENDIX B 


\section{LIST OF FIGURES}

FIGURE 1: DENSITY VARIATION AS FUNCTION OF THN PERCENTAGE FOR THN-C ${ }_{12} \mathrm{H}_{26}$ MIXTURE $\quad 42$

FIGURE 2: DENSITY VARIATION AS FUNCTION OF IBB PERCENTAGE FOR IBB-C $\mathrm{C}_{12} \mathrm{H}_{26}$ MIXTURE 43

FIGURE 3: DENSITY VARIATION AS FUNCTION OF THN PERCENTAGE FOR THN-IBB MIXTURE 44

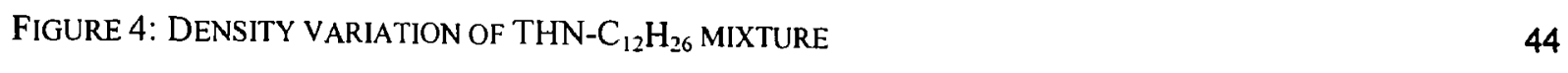

FIGURE 5: DENSITY VARIATION OF IBB-C $\mathrm{C}_{12} \mathrm{H}_{26}$ MIXTURE $\quad 45$

FIGURE 6: DENSITY VARIATION OF THN-IBB MIXTURE

FIGURE 7: TWO-DIMENSIONAL POROUS MEDIA MODEL AND BOUNDARY CONDITIONS 50

FIGURE 8: MESH SENSITIVITY ANALYSIS $\quad \mathbf{5 8}$

FIGURE 9: THN-DODECANE: STREAMLINE CONTOURS

FIGURE 10: THN-DODECANE: VELOCITY ALONG THE CENTER OF THE CAVITY

FIGURE 11: IBB-DODECANE: STREAMLINE CONTOURS

FIGURE 12: IBB-DODECANE: VELOCITY ALONG THE CENTER OF THE CAVITY $\quad 62$

FIGURE 13: THN-IBB: STREAMLINE CONTOURS $\quad 63$

FIGURE 14: THN-IBB: VELOCITY ALONG THE CENTER OF THE CAVITY

FIGURE 15: THN-DODECANE: HORIZONTAL DENSITY DISTRIBUTION

FIGURE 16: THN-DODECANE: VERTICAL DENSITY DISTRIBUTION

FIGURE 17: IBB-DODECANE: HORIZONTAL DENSITY DISTRIBUTION $\quad 67$

FIGURE 18: IBB-DODECANE: VERTICAL DENSITY DISTRIBUTION

FIGURE 19: THN-IBB: HORIZONTAL DENSITY DISTRIBUTION

FIGURE 20: THN-IBB: VERTICAL DENSITY DISTRIBUTION

FIGURE 21: THN-DODECANE: CONCENTRATION CONTOURS FOR PERMEABILITY RANGING FROM 0.001 MD TO $\begin{array}{ll}10,000 \mathrm{MD} & 72\end{array}$

FIGURE 22: IBB-DODECANE: CONCENTRATION CONTOURS $\quad 73$

FIGURE 23: THN-IBB: CONCENTRATION CONTOURS

FIGURE 24: SEPARATION RATIO AS A FUNCTION OF THE PERMEABILITY $\quad 76$

FIGURE 25: CHARACTERISTIC TIMES AS A FUNCTION OF THE PERMEABILITY $\quad \mathbf{7 8}$

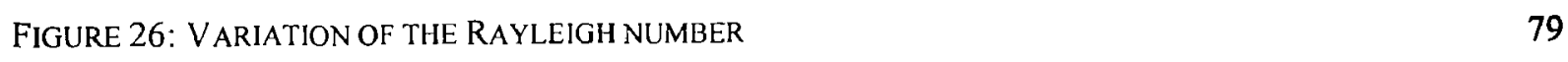

FIGURE 27: TWO-DIMENSIONAL HETEROGENEOUS POROUS MEDIA CAVITY; THE LEFT REPRESENTS THE HORIZONTAL FRACTURE CASE, AND THE RIGHT REPRESENTS THE VERTICAL FRACTURE CASE $\quad \mathbf{8 3}$

FIGURE 28: STREAMLINE CONTOURS FOR UN-FRACTURED AND FRACTURED CASE $\quad \mathbf{8 4}$

FIGURE 29: STREAMLINE CONTOURS FOR HORIZONTAL FRACTURE $\quad \mathbf{8 5}$

FIGURE 30: HORIZONTAL FRACTURE: VELOCITY VERTICALLY INSIDE THE CAVITY

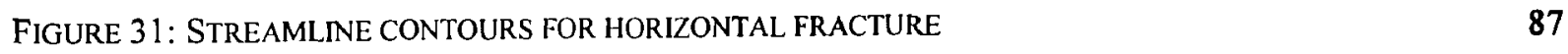

FIGURE 32: VERTICAL FRACTURE: VELOCITY HORIZONTALLY INSIDE THE CAVITY

FIGURE 33: HORIZONTAL FRACTURE CASE: CONCENTRATION CONTOURS 99 
FIGURE 34: VERTICAL FRACTURE CASE: CONCENTRATION CONTOURS

FIGURE 35: SORET COEFFICIENT FOR HORIZONTAL FRACTURE CASE

FIGURE 36: SORET COEFFICIENT FOR THE VERTICAL FRACTURE CASE 


\section{LIST OF TABLES}

TABLE 1: THE CRITICAL PROPERTIES OF PURE COMPONENTS

TABLE 2: THE DENSITY CALCULATION AT $P=1$ BAR AND $T=298 \mathrm{~K}$

TABLE 3: THEORETICAL AND EXPERIMENTAL MOLECULAR DIFFUSION COEFFICIENTS FOR THREE BINARY MIXTURES

TABLE 4: THEORETICAL AND EXPERIMENTAL THERMAL DIFFUSION COEFFICIENTS FOR THREE BINARY MIXTURES

TABLE 5: THEORETICAL AND EXPERIMENTAL THERMAL DIFFUSION COEFFICIENTS FOR THE TERNARY SYSTEM

TABLE 6: THERMAL DIFFUSION COEFFICIENTS CALCULATED USING THE LINEAR COMBINATION

TABLE 7: MAXIMUM SEPARATION OBTAINED BY ANALYTICAL EXPRESSION AND NUMERICAL SIMULATION 76 


\section{NOMENCLATURE}

\begin{tabular}{|c|c|}
\hline$c_{p}$ & Heat capacity \\
\hline$c_{k}$ & Component fraction \\
\hline $\mathrm{D}^{\mathrm{M}}$ & Mass diffusion coefficient $\left(\mathrm{m}^{2} / \mathrm{s}\right)$ \\
\hline$\widetilde{\mathrm{D}}^{\mathrm{M}}$ & The molecular diffusion coefficient tensor $\left(\mathrm{m}^{2} / \mathrm{s}\right)$ \\
\hline$D^{P}$ & Pressure diffusion coefficient $\left(\mathrm{m}^{2} / \mathrm{s} . \mathrm{Pa}\right)$ \\
\hline$\widetilde{D}^{p}$ & The pressure diffusion coefficient tensor $\left(\mathrm{m}^{2} / \mathrm{s} . \mathrm{Pa}\right)$ \\
\hline $\mathrm{D}^{\mathrm{T}}$ & Thermal diffusion coefficient $\left(\mathrm{m}^{2} / \mathrm{s} . \mathrm{K}\right)$ \\
\hline$\widetilde{\mathrm{D}}^{\mathrm{T}}$ & The thermal diffusion coefficient tensor $\left(\mathrm{m}^{2} / \mathrm{s} . \mathrm{K}\right)$ \\
\hline$\breve{D}_{i}^{T}$ & Calculated thermal diffusion coefficient $\left(\mathrm{m}^{2} / \mathrm{s} . \mathrm{K}\right)$ \\
\hline$D_{T}^{i}$ & Experimental thermal diffusion coefficient $\left(\mathrm{m}^{2} / \mathrm{s} . \mathrm{K}\right)$ \\
\hline $\overrightarrow{\mathrm{J}}$ & The tensor of all component mass flux $\left(\mathrm{kg} / \mathrm{m}^{2} . \mathrm{s}\right)$ \\
\hline $\mathrm{J}_{1}$ & Mass flux of component 1 in the binary mixture $\left(\mathrm{kg} / \mathrm{m}^{2} . \mathrm{s}\right)$ \\
\hline $\mathrm{k}$ & Thermal conductivity (W/m. K) \\
\hline $\overrightarrow{\nabla \mathrm{P}}$ & The vector of pressure gradient $(\mathrm{Pa})$ \\
\hline $\mathrm{q}$ & Separation ratio \\
\hline $\mathrm{S}_{\mathrm{T}}$ & Soret Coefficient $(1 / K)$ \\
\hline $\mathrm{t}$ & Time (s) \\
\hline $\overrightarrow{\nabla T}$ & The vector of temperature gradient $(\mathrm{K})$ \\
\hline $\mathrm{u}$ & Velocity in $\mathrm{x}$-direction $(\mathrm{m} / \mathrm{s})$ \\
\hline $\mathrm{v}$ & Velocity in $\mathrm{y}$-direction $(\mathrm{m} / \mathrm{s})$ \\
\hline 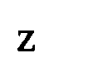 & Compressibility factor \\
\hline
\end{tabular}

GREEK LETTERS

$\begin{array}{ll}\alpha_{p} & \text { Pressure relaxation factor } \\ \alpha_{T} & \text { Thermal diffusion coefficient in a binary mixture }\left(\mathrm{m}^{2} / \mathrm{s}\right) \\ \beta_{C} & \text { Volumetric concentration expansion coefficient }\end{array}$




$\begin{array}{ll}\beta_{\mathrm{p}} & \text { Compressibility coefficient } \\ \beta_{\mathrm{T}} & \text { Coefficient of thermal expansion }(1 / \mathrm{K}) \\ \kappa & \text { Permeability }\left(\mathrm{m}^{2}\right) \\ \mu & \text { Dynamic viscosity, or chemical potential }(\mathrm{kg} / \mathrm{m} \cdot \mathrm{s}) \\ \rho & \text { Density }\left(\mathrm{kg} / \mathrm{m}^{3}\right) \\ \tau_{\mathrm{f}} & \text { Flow characteristic time (s) } \\ \tau_{\text {th }} & \text { Thermal characteristic time (s) } \\ \varphi & \text { Porosity of the porous medium } \\ \phi & \text { Relative error } \\ \psi & \text { Volume fraction of molecules }\end{array}$

\section{SUBSCRIPTS AND SUPERSCRIPTS}

$\begin{array}{ll}0 & \text { Reference state } \\ \text { F } & \text { Firoozabadi model } \\ \text { H } & \text { Haase model } \\ \text { i } & \text { Component i } \\ \text { K } & \text { Kempers model } \\ \text { m } & \text { Mean, or porous medium } \\ \text { s } & \text { Solid }\end{array}$




\section{CHAPTER 1: INTRODUCTION}

The thermal diffusion process, also known as the Soret effect, is the tendency of a convection-free mixture to separate under a temperature gradient. For binary mixtures, the Soret effect is measured by the Soret coefficient, $\mathrm{S}_{\mathrm{T}}$, which is the ratio of the thermal diffusion coefficient, $D^{T}$, to the molecular diffusion coefficient $D^{M}$. However, for multicomponent mixtures, the thermal diffusion coefficient is more commonly used as a measure of the Soret effect. The Soret effect is an important phenomenon for the study of the compositional variation in hydrocarbon reservoirs. It also plays a crucial role in the hydrodynamic instability of mixtures, mineral migrations and mass transport in living matter. In low-pressure gaseous mixtures and ideal liquid mixtures, the magnitude of the thermal diffusion coefficient may be small. Conversely, in non-ideal liquid mixturesparticularly, close to the critical points-the thermal diffusion coefficient becomes large and strongly depends on the energetic interactions, the size and shape of the molecules and thermodynamic conditions.

For years, thermal diffusion has triggered active research in both theoretical and experimental studies. A large part of such research activities is on thermal diffusion in porous medium by researchers such as Nield et al. [1] and Ingham et al. [2, 3]. A porous medium is usually defined as material that consists of a solid matrix with an interconnected void. The void-also known as pores-allow for the flow of one or more fluids through the material. The distribution of the pores in a natural porous medium with respect to the size and shape is irregular.

This chapter gives a detailed literature review, which introduces a variety of techniques for measuring the Soret coefficient. It also discusses the mathematical and numerical methods for the simulation of the Soret effect in both free and porous media. In addition, this chapter reviews the techniques for evaluating transport coefficients. Finally, it presents the modeling of fractures for flow and transport in porous media. 


\subsection{LITERATURE REVIEW}

In general, the Soret coefficient is evaluated based on the component differentiation in the cell. Several ground based techniques have been developed to measure the Soret coefficient. Two techniques are commonly used for binary mixtures with high-melting points. (1) The long capillary technique, where the samples are processed in capillary tubes placed in a gradient furnace. The liquid vein is then quenched once a steady state separation is attaine. (2) The shear cell technique, where there is a superposition of discs with veins running through them. The temperature gradient is created between the bottom disc and the very top disc in the shear cell. Once the process is completed, discs are rotated around the central axis in order to divide the liquid veins, hence isolating the sections for further analysis. The benefit of this technique is that it does not require the quenching process, which can cause volume contractions, convection, and phase segregation and hence influence the values obtained.

To measure the Soret coefficients of binary systems with low melting points, numerous methods have been developed. The flow cell, for example, is one technique in which the separation process occurs in a very thin flowing layer in the Poiseuille regime. A sharp edge at the outlet separates the flow in upper and lower halves. The difference of concentration between the two samples is analyzed by using refractometry or densitometry. Another commonly used technique is the Benard cell configuration. Here, the flow is heated from below and the onset of convection is determined by the SchmidtMilverton plot technique, Schmidt and Milverton [4]. Additionally, the diaphragm cell is a technique in which a porous medium is used to separate the flow into two chambers at different temperatures. Finally, the thermogravitation column is a common technique, where a temperature gradient is imposed horizontally on a vertical channel. This creates a differential accumulation of different species at the top and bottom. The Soret coefficient is then evaluated from the obtained separation.

Optical techniques were explored in the Soret coefficient measurement such as, the Thermal Diffusion Forced Rayleigh Scattering (TDFRS) technique by Kohler et al. [5], 
and the Laser-Doppler Velocimetry (LDV) technique by Kohler et al. [6]. According to the principle of TDFRS, a grating created by the interference of two laser beams is written in a sample. A small amount of dye present in the sample, converts the intensity grating into a temperature gradient. This in turn causes a concentration gradient by the effect of thermal diffusion. Both gratings contribute to a combined refractive index grating, which is read out by diffraction of a third laser beam. Upon analysis of the time dependent diffraction efficiency, the transport coefficients are then obtained. In the LDV technique, the experimental cell consists of two horizontal copper plates that are maintained at different yet constant temperatures. The imposed temperature difference is then gradually increased in small steps. Enough time is allowed between two successive temperature increments to allow steady state to establish. The time dependent velocity amplitude is recorded, and, on the onset of convection, the critical temperature difference and the dimensional frequency corresponding to the oscillation of the velocity amplitude is used to calculate the Soret coefficient. Kohler et al. [6] also discovered that the LDV technique is more accurate than the Schmidt-Milverton plot technique in measuring the Soret coefficient.

Convection has a major influence on the accuracy of the Soret measurements. Utilization of porous media might help in reducing the distortion caused by the convection because the fluid moves slowly in porous media. Costeseque et al. [7] conducted experiments in both a free fluid and a porous medium. They utilized a cell with two horizontal steel plates maintained at different temperatures in order to create a vertical temperature gradient. Six couples of small holes along the horizontal sides were used to retrieve small liquid samples. These couples were then analyzed using a high-resolution refractometer to determine the concentration difference. Two experiments were conducted in the same cell with the same thermal gradient; the first experiment representing the free fluid case and the second representing the porous medium case. It was found that, when the thermal conductivities of the fluid and solid matrix are of the same magnitude, the Soret coefficients do not differ significantly from the case of the porous medium to that of the free fluid. These findings indicate that the contribution of the solid matrix in a porous medium on the two mass fluxes-namely the isothermal mass flux caused by the gradient 
of chemical potential, and the thermodiffusive mass flux caused by the temperature gradient-is the same as those in the free fluid. Therefore, it was concluded that the Soret coefficient is the same in a free fluid as in a porous medium.

Several researchers have published values of the Soret coefficient for organic molecules, polymers, and even electrolytes solutions. However, few data are available from various techniques on the same systems. In summer 1999, research groups from various universities started a ground-based measurement campaign. The goal was to establish a reliable database of the Soret coefficient for three binary mixtures composed of dodecane, isobutylbenzene, and 1,2,3,4-tetrahydrophtalene. As well, there was a clear desire to establish benchmark values for the Soret, thermal diffusion, and diffusion coefficients so that they could be used to compare Earth results with results obtained through other means such as experiments in microgravity or numerical simulation. The values obtained from independent researchers, were in strong agreement with each other, Platten et. al. [8]; their differences did not exceed more than 7\%. The Soret coefficient for three binary mixtures of (i) 1,2,3,4 Tetrahydronaphtalene - Dodecane with a mass fraction of 50\% for each component, (ii) 1,2,3,4 Tetrahydronaphtalene - Isobutylbenzene with a mass fraction of $50 \%$ for each component, and (iii) Isobutylbenzene - Dodecane with a mass fraction of $50 \%$ for each component were found to be $(9.5 \pm 0.5) \times 10^{-3} 1 / \mathrm{K}$, $(3.3 \pm 0.3) \times 10^{-3} 1 / \mathrm{K}$ and $(3.9 \pm 0.1) \times 10^{-3} 1 / \mathrm{K}, \quad$ respectively at the mean temperature of $25^{\circ} \mathrm{C}$.

Platten et. al. [9] also measured the thermodiffusion coefficients of a ternary hydrocarbon mixture by utilizing the thermogravitational column technique. The ternary mixture consists of one normal alkene and two aromatics (1,2,3,4 tetrahydronaphtalene-dodecaneisobutylbenzene) with equal mass fractions for all components at a mean temperature of $25^{\circ} \mathrm{C}$. The concentration variations in the column were measured using refractometry, and the density was measured by a densimeter. Platten et al. [9] correlated the thermodiffusion coefficients in the ternary mixture with those in the three binary mixtures. 
Despite the fact that the transport coefficient data for binary mixtures is sparsely available, it is already known that the variation of hydrocarbon reservoirs - a non-ideal multi-component mixture system-cannot be determined using results obtained from binary mixtures since diffusion coefficients may significantly differ in multi-component mixtures. Furthermore, the sign of thermal diffusion factors is immaterial in defining the direction of the component segregation in the hot or cold regions for multi-component mixture systems. Moreover, for non-ideal multi-component mixtures, the formalism is more complicated and the interaction theory of all chemical species present in the mixture is still not fully developed. More work is being performed presently to understand the species behaviour in systems far from concentration equilibrium and to determine the diffusion process in multi-component mixtures.

Shukla and Firoozabadi [10] developed a model for thermal diffusion factors in multicomponent non-ideal mixtures. This model was based on the thermodynamics of irreversible processes where the effects of both equilibrium and non-equilibrium properties are incorporated. The equilibrium properties, such as partial internal energies and fugacities, were estimated using the volume-translated Peng-Robinson equation of state. On the other hand, the non-equilibrium properties, such as viscosity, were accounted for by incorporating the energy of viscous flow. The model was validated with the measured compositional data for a ternary mixture $\mathrm{nC}_{21} / \mathrm{nC}_{16} / \mathrm{nC}_{12}$. They further predicted the thermal diffusion behaviour for a six-component mixture of $\mathrm{C}_{1} / \mathrm{C}_{3} / \mathrm{nC}_{5} / \mathrm{nC}_{10} / \mathrm{nC}_{16} / \mathrm{C}_{2}$ at various temperatures and pressures and found that when the system was far from the critical point, the thermal diffusion factor was not sensitive to temperature or pressure. Firoozabadi et al. [11] further examined the performance of this model for three different binary systems; the hydrocarbon system, i.e., $C_{1} / C_{3}, C_{1} / C_{4}$, $\mathrm{C}_{7} / \mathrm{C}_{12}$ and $\mathrm{C}_{7} / \mathrm{C}_{16}$, the non-hydrocarbon system, i.e., $\mathrm{Ar} / \mathrm{CO}_{2}, \mathrm{~N}_{2} / \mathrm{CO}_{2}, \mathrm{H}_{2} / \mathrm{N}_{2}$, and $\mathrm{H}_{2} / \mathrm{CO}_{2}$, and the hydrocarbon-nonhydrocarbon system, i.e., $\mathrm{C}_{1} / \mathrm{N}_{2}$ and $\mathrm{C}_{1} / \mathrm{CO}_{2}$. These three systems were used to model the binary mixtures of reservoir fluids. The model was compared with experimental data and two other models: the Rutherford and Kempers models. When detailed comparison is made between the theoretical results and experimental data, the Firoozabadi model is superior in describing experimental results 
for the thermal diffusion coefficients in binary mixtures. In particular, the sign of thermal diffusion coefficient is consistent with the experimental data.

The thermal diffusion in packed columns (TPC) refers to an instance when a porous medium is present in the column between two thermal sources. The advantage of studying the porous configuration is that it allows the investigation of the thermogravitation effect in natural systems, which tends to exist in porous media such as oil reservoirs. The thermogravitation effect describes the coupling between natural convection and the diffusion processes. Furthermore, this mechanism is also believed to be capable of efficiently redistributing the hydrocarbon species in a porous medium over a geological time scale. Additionally, the porous packing governs the coupling conditions between the vertical and horizontal transport. Since the mass separation in a thermogravitational column depends on the balance between two rates-the rate of mass flux (due to the basic thermal diffusion effect) in the horizontal direction and the convection velocity in the vertical direction-anything that decreases the convection velocity, such as packing, increases the relative importance of the horizontal flux, and thus increases the steady state mass separation in the batch column. A study by Lorenz and Emery [12] shows that the maximum magnitude of separation or the optimum coupling between buoyancy and thermal diffusion is obtained at a particular value of the permeability. They also found that the packed column offered the advantage of using columns constructed with fewer intricacies.

Jamet et al. [13] presented a computer model of thermal diffusion in packed columns to determine the governing transport coefficients, especially the thermal diffusion coefficient. They found that due to the intricacy of the coupled processes occurring in the TPC column, the diffusion coefficient could not be ascribed as an a priori value. Rather, the diffusion coefficient needed to be considered as an implicit parameter. Based on this methodology, a model that utilized hydrodynamic dispersion theory was created. This model provided values that were closer to the experimental values. Therefore, they concluded that implicit approach for the diffusion coefficient could in fact reduce the deviation between the experimental and numerical methods. 
Benano-Melly et al. [14] investigated numerically the phenomenon of thermogravitation in binary fluid mixtures. In their study, a two-dimensional cell with two vertical walls set at constant yet different temperatures was used. The binary mixture filling the cell porous medium was initially homogenous, and the two-dimensional cell's boundary condition was no flow and no mass flux through the cell walls. The flow analysis showed that the mixture undergoes convection due to the presence of a thermal gradient, with higher convection velocity near the cell vertical sides. When the solutal Rayleigh number is high enough, and the Soret number is positive, the solutal and thermal buoyancy forces combine their actions to enhance convection. On the other hand, when the Rayleigh number is high and the Soret number is negative, the counteracting solutal and thermal buoyancy forces weaken the convection. It was also observed that the solutal buoyancy force had negligible, or no effect, on the convection for solutal Rayleigh numbers lower than 0.1. Benano-Melly et al. [14] further investigated the solute migration and distribution behavior and found that the amplitude of separation mainly depends on the thermal Rayleigh number; a decreased Lewis number results in a decreasing separation ratio.

The thermal diffusion process can occur in both liquid and gaseous mixtures. Studies based on the thermodynamics of irreversible processes have shown that thermal diffusion, along with natural convection, can in fact have a significant effect on the compositional variation in the horizontal and vertical directions in hydrocarbon reservoirs. According to these studies, thermal diffusion can either enhance or weaken the separation in mixtures. Riley and Firoozabadi [15] presented a model to investigate the effects of natural convection and diffusion (thermal, pressure and fickian) on a singlephase binary mixture of Methane and nButane in a horizontal cross-sectional reservoir in the presence of a prescribed linear temperature field. The compositional distribution in the reservoir under both horizontal and lateral heating conditions was carefully examined. It was found that increasing the permeability increases the horizontal compositional variation. A local maximum and/or minimum value exists in the compositional gradient as a function of the permeability. As the permeability approaches infinity, the compositional gradient lessens monotonically to zero. The right and left boundaries of the 
reservoir were noticed to have a significant boundary layer characteristic. As the permeability increases, the thickness of the boundary layer decreases. Although there is a rapid variation in the compositional gradients within these boundaries, the effect of the boundary in the larger remaining region in between is insignificant.

The evaluation of transport coefficients by ground-based techniques is difficult. In particular, in multi-component systems, such evaluation is complex since the number of couplings between the thermal and composition transport-and consequently the number of transport coefficients to be known-increases rapidly. When convection is coupled to thermal and solute transport, the phenomenon is known as double diffusion convection. One possible remedy is to minimize the buoyancy by performing experiments in microgravity on free flying platforms, such as the International Space Station. In microgravity conditions, the convection induced by gravity is eliminated. Chacha et al. [16] simulated the double diffusion process under several thermal boundary conditions in order to better understand the influence of these variations during a microgravity experiment. These included radiating horizontal walls, smooth change in the lateral thermal boundary condition, and, even more drastic, the abrupt change in the lateral thermal boundary condition. They observed that when there is a strong convection, secondary recirculation eddies form along the corners of the cavity and a clockwise rotating cell is present in the center of the cavity. This formation indicates the strength of convection inside the cavity. The temperature profile near the hot and cold walls was distorted due to the presence of convection. As well, the heat transfer due to convection is stronger and faster than the heat transfer due to conduction. They therefore concluded that the transport of heat in the boundary layers, as well as in normal gravity, is driven by the convective flow. It was observed that in the case of radiating horizontal walls, a stronger radiative heat loss, or, a high emissive or a very weak temperature difference between the end walls, could possibly lead to the break up of the entire cavity into different thermodiffusion sub-cells. This would hence result in greater uncertainties in the Soret coefficient measurements. Further, it was observed, in the event of power outage during an experiment in space, that the system responds with some delay to the modification of the boundary conditions. 
Due to the absence of buoyancy-induced convection, the experiments on the microgravity environment aboard platforms, such as the International Space Station (ISS), may lead to accurate measurement of the Soret coefficient for multi-component mixtures. However, like other space laboratories, the ISS experiences a steady and/or residual time dependent acceleration called gravity jitter or g-jitter. Such acceleration may be caused by the movement of the crew, thruster firing for altitude adjustment, or, operation of equipment. Therefore, it is important to consider the effect of g-jitter in the diffusion-dominated fluid science experiments on the ISS. Theoretical work in this area has been reported by several authors such as Gershuni and Zhukhovitsky [17], and Savino et al. [18] just to name a few. Recently, Chacha et al. [19] investigated the role of thermal diffusion phenomena on compositional variation in a binary mixture of Methane and n-Butane in the presence of g-jitter. They found that the presence of g-jitter causes mixing and overcomes the Soret effect in a cavity, therefore making it difficult to measure the Soret coefficient accurately in space.

The presence of fractures in porous medium is often associated with high containment or productivity. Therefore, the fluid flow through fractures is a process that has importance for many areas of the geosciences, ranging from ground-water hydrology to oil extraction. Research on fluid flow in fractures and in fractured porous media has a history that spans nearly four decades, and several conceptual models have been developed for properly modeling fractured formations. Fundamentally, each model can be distinguished on the basis of the storage and flow capabilities of the porous medium and the fracture. The storage characteristics are associated with porosity, and the flow characteristics are associated with permeability. The most recent and widely used model is the multiporosity/multi-permeability conceptual model. The multi-porosity/multi-permeability models are based on an idealized flow medium consisting of a primary porosity, created by deposition and lithification, and a secondary porosity, created by fracturing, jointing, or dissolution. The porous medium and the fractures are envisioned as two separate but overlapping continua. Fluid mass transfer between porous media and fractures occur at the fracture-porous medium interface. 
Sahimi et al. [20] analyzed fluid flow in fractured reservoirs and developed a numerical model based on the basic physics of fluid flow in the Earth. A near field model was utilized where flow occurs in a single fracture and porous medium exchange is possible. A finite difference approximation was used. The pressure and the scalar components of velocity in the partial difference Stokes equation were represented in discrete forms for both space and time through equivalent finite difference equations, and a forward difference scheme was used. The advantage of the model is that it allows the simulation on a micro-scale as opposed to convectional macro-scale simulation. The advantage of the micro-scale (pore scale) simulation is that it allows for the analysis of variation of fluid velocity from point to point within the fractured porous media, and hence the motion of the fluid contributes to the understanding of fluid flow throughout the fractured porous medium. It was found that the micro-scale simulation not only produced more realistic and accurate results than the macro-scale simulation, but it was also particularly useful in identifying high potential production zones in the fractured reservoirs. Furthermore, the micro-scale simulation can be used as a tool to analyze flow in complicated networks of fractures to understand the pattern of flow and thus step forward towards more accurate ways of reservoir simulation.

Alboin et al. [21] modelled fractures as interfaces for flow and transport in porous media. Contrary to many studies in which the contrast in permeabilities is of such an order that the flow outside of fracture is neglected, the Alboin et al. [21] study considered the case where the exchange between the fractures and the surrounding rock is significant. In other words, treating fractures as interfaces as opposed to barriers. The fracture was modelled as a porous medium with high permeability compared to that in the subdomains surrounding the fracture. In the sub-domains, as well as in the fracture, the flow was assumed to be incompressible and to satisfy Darcy's law. A pressure drop drove flow in the fracture between the two extremities of the fractures. Two cases were considered, a symmetric case and a non-symmetric case. The pressures on the left and on the right boundaries of the domain were equal, so that the flow is driven only by the fracture. The second case considered a pressure drop from the left boundary to the right boundary. Hence, the flow is a combination of the flow in the fracture and that going from left to 
right in the rest of the porous media. The results showed that when pressure was equal, there was very little flow interaction between the fracture and the rest of the porous medium. In particular, it was evident that not much fluid was coming out of the fracture or going back into it. In the non-symmetric case, it was noted that the most of the flow was attracted to the fracture; however, there was some flow from left to right through the fracture. Therefore, simple numerical experiments illustrated actual flow and transport interactions between the fractures and the rest of the porous media.

There are large numbers of publications that examine the effect of fractured formations by utilizing contrast in permeabilities or, in other words, heterogeneity in porous media. However, these publications investigate the fluid flow without taking into account the phenomenon of thermodiffusion in fractured porous um Jiang et al. [22] investigated the effect of thermodiffusion in a heterogeneous porous media. In the numerical experiment, a vertical cavity was filled with a binary hydrocarbon mixture of Methane and n-Butane, and was laterally heated. The fracture was modelled by varying the permeability of the upper domain while the permeability of the lower domain remained constant. The porosity was constant and the same in both domains. The unique feature of this numerical experiment is that not only the Soret effect was investigated in a heterogeneous porous medium but also the Soret coefficient was not fixed as constant; rather it was calculated at each point of the grid as a function of temperature, pressure, and concentration. It was noted that, when the permeability in the upper cavity was less than the permeability in the lower domain the convection in the lower domain became dominant. However, when the permeability in the upper cavity was higher than lower cavity, the convection flow in the upper part dominates, and the flow pattern of the lower portion is distorted at the interface area. It was also noticed that the concentration in the upper part varied drastically; however, in the lower part, the concentration distribution was approximately the same. The variation of separation ratio in the upper part of the heterogeneous porous medium was compared with the separation ratio for the homogenous case, where the whole cavity is specified to have the same permeability. The maximum separation ratio was found identical for both cases. However, the overall separation ratio was higher in the heterogeneous porous media than in the homogenous porous media. 


\subsection{RESEARCH OBJECTIVES}

Hydrocarbon mixtures in porous media, such as oil reservoirs, are profoundly influenced by thermal diffusion. Even after nearly a century of research, theoretical studies have produced only a few models to explain this phenomenon. Furthermore, only a handful of models produce acceptable results and only the Firoozabadi model accurately predicts the mechanism in non-ideal multicomponent mixtures. The main objective of this research is to verify the application of the Firoozabadi model by comparing results for three binary mixtures consisting of n-Dodecane $\left(\mathrm{C}_{12} \mathrm{H}_{26}\right)$, Isobutylbenzene (IBB), and Tetrahydronaphtalene (THN), with benchmark experimental values. In addition to that, the thermal diffusion coefficients for a ternary hydrocarbon mixture are calculated and compared with experimental data. The thermal and molecular diffusion coefficients for three binary mixtures are used to correlate and estimate the thermal diffusion coefficients for the ternary mixture. A two dimensional numerical model is used to investigate the thermo-solutal convection in porous media. A rectangular cavity is laterally heated, and the porosity of the porous medium in the cavity is varied to gain understanding of thermal diffusion phenomena in underground oil reservoirs. Finally, a dual porosity-dual permeability model is utilized to simulate vertical and horizontal fracture in underground

oil reservoirs, and to investigate fluid mechanics and thermal diffusion in fractures and cracks. 


\section{CHAPTER 2: FUNDAMENTAL THEORY}

\subsection{THEORETICAL MODELS OF THERMAL DIFFUSION}

Although the thermal diffusion coefficient can be measured through different techniques, the measurement condition has to be controlled strictly to avoid the possible occurring effect of thermal convection, which is very difficult in ground conditions. For years, research has endeavoured to generate reliable thermal diffusion coefficient models. Several theoretical approaches have been proposed for binary mixtures with various degrees of success. These include the phenomenological theory of irreversible thermodynamics by Haase [23], the kinetic theory of irreversible thermodynamics by Rutherford [24], Dougherty and Drickamer [25] and Shukla and Firoozabadi [10], the elementary transition state theory by Mortimer and Eyring [26], the kinetic theory of dense hard spheres and its revisions by De Haro et al.[27] and Kincaid et al. [28], the partial excess enthalpy and activity coefficient by Guy [29], the Brownian motion and heat of transport by Bearman et al. [30], and the maximization of the partition function of two idealized bulbs by Kempers [31]. It should be kept in mind that in all of the above mentioned models, partial molar properties--derived from the equation of state (EOS) are required. Therefore, for any particular mixture, the accuracy of the model not only relies on the model itself, but also on both the EOS of choice and the numerical method utilized in the calculation. Below, three models are listed and described. For further reading on other models, refer to the original papers.

\subsubsection{Haase Model}

The Haase [23] model is based on the phenomenological approach. Such an approach uses the phenomenological equations of irreversible thermodynamics to derive the following equation for binary systems in terms of the net heat of transport and chemical potential: 


$$
\alpha_{T}=\frac{Q_{2}^{\cdot}-Q_{1}^{\cdot}}{x_{1}\left(\partial \mu_{1} / \partial x_{1}\right)}
$$

where $\alpha_{T}$ is the thermal diffusion factor in a binary mixture, $x_{1}$ is the mole fraction of component $1, \mu_{1}$ is the chemical potential of component 1 , and $Q_{i}^{*}$ is the net heat of transport of component $i(i=1,2)$. The thermal diffusion factor is usually applied in experimental analysis and can be obtained based on measured data. The Soret coefficient can be obtained by dividing the thermal diffusion factor by the temperature in Kelvin.

The net heat of transport is then interpolated with the molar enthalpy in a mass conserved system. With this assumption, the net heat of transport is expressed in the following format with the thermostatic values of enthalpy:

$$
Q_{2}^{*}-Q_{i}^{*}=\frac{M_{1} M_{2}}{M_{1} x_{1}+M_{2} x_{2}}-\left(\frac{H_{2}}{M_{2}}-\frac{H_{1}}{M_{1}}\right)
$$

where $H_{i}$ is the partial molar enthalpy of component $i(i=1,2)$ and $M_{i}$ is the molecular weight of component $i(i=1,2)$. The expression for the thermal diffusion factor in the Haase model will then become

$$
\alpha_{T}^{H}=\frac{M_{1} H_{2}-M_{2} H_{1}}{\left(M_{1} x_{1}+M_{2} x_{2}\right) x_{1}\left(\partial \mu_{1} / \partial x_{1}\right)}
$$

For ideal gas/liquid at a reference temperature, equation (3) gives

$$
\alpha_{\mathrm{T}}^{0}=\frac{\mathrm{M}_{1} \mathrm{H}_{2}^{0}-\mathrm{M}_{2} \mathrm{H}_{1}^{0}}{\left(\mathrm{M}_{1} \mathrm{x}_{1}+\mathrm{M}_{2} \mathrm{x}_{2}\right) \mathrm{RT}}
$$

where $\mathrm{R}$ is the gas constant. Then, the Haase model may be re-written, with regard to a reference state, as follows: 


$$
\alpha_{\mathrm{T}}^{\mathrm{H}}=\frac{\alpha_{\mathrm{T}}^{0} \mathrm{RT}\left(\mathrm{M}_{1} \mathrm{x}_{1}+\mathrm{M}_{2} \mathrm{x}_{2}\right)+\mathrm{M}_{1}\left(\mathrm{H}_{2}-\mathrm{H}_{2}^{0}\right)-\mathrm{M}_{2}\left(\mathrm{H}_{1}-\mathrm{H}_{1}^{0}\right)}{\left(\mathrm{M}_{1} \mathrm{x}_{1}+\mathrm{M}_{2} \mathrm{x}_{2}\right) \mathrm{x}_{1}\left(\partial \mu_{1} / \partial \mathrm{x}_{1}\right)}
$$

\subsubsection{Kempers Model}

The Kempers [31] model is based on a statistical description of a non-equilibrium twobulb system, which is similar to a system with a fixed volume at a uniform pressure without external forces. This assumption leads to the expression of the net heat of transport as follows:

$$
Q_{2}^{*}-Q_{1}^{*}=\frac{V_{1} V_{2}}{V_{1} x_{1}+V_{2} x_{2}}\left(\frac{H_{2}}{V_{2}}-\frac{H_{1}}{V_{1}}\right)
$$

where $V_{i}$ is the partial molar volume of component $i$, and $H_{i}$ is the partial molar enthalpy of component $i(i=1,2)$. Combining equations (1) and (6), the thermal diffusion factor in the Kempers model is then given by:

$$
\alpha_{T}^{K}=\frac{V_{1} H_{2}-V_{2} H_{1}}{\left(V_{1} x_{1}+V_{2} x_{2}\right) x_{1}\left(\partial \mu_{1} / \partial x_{1}\right)}
$$

\subsubsection{Firoozabadi Model}

Based on the thermodynamics of irreversible processes, Firoozabadi et al. [10, 15] presented a model for binary mixtures of reservoir fluids. In this model, the net heat of transport is related to the energy of detaching a molecule from its neighbours in the region of the mixture, and to the energy given up in that region when one molecule fills a hole. This energy is expressed in terms of the partial molar internal energy and the ratio of the energy of vaporization to the energy of viscous flow of the component. The net heat of transport is given by 


$$
\left\{\begin{array}{l}
\mathrm{Q}_{1}^{*}=\mathrm{W}_{\mathrm{H} 1}-\psi_{1}\left(\mathrm{x}_{1} \mathrm{~W}_{\mathrm{H} 1}+\mathrm{x}_{2} \mathrm{~W}_{\mathrm{H} 2}\right) \\
\mathrm{Q}_{2}^{*}=\mathrm{W}_{\mathrm{H} 2}-\psi_{2}\left(\mathrm{x}_{1} \mathrm{~W}_{\mathrm{H} 1}+\mathrm{x}_{2} \mathrm{~W}_{\mathrm{H} 2}\right)
\end{array}\right.
$$

with

$$
\left\{\begin{array}{l}
\mathrm{W}_{\mathrm{H} 1}=-\mathrm{U}_{1} / \tau_{1} \\
\mathrm{~W}_{\mathrm{H} 2}=-\mathrm{U}_{2} / \tau_{2}
\end{array}\right.
$$

where $U_{i}$ is the partial molar internal energy of component $i(i=1,2)$, and $\tau_{i}$ is the ratio of the energy of vaporization, $\Delta U^{\text {vap }}$, and the energy of viscous flow, $\Delta U^{\text {visc }}$, i.e.,.

$$
\tau_{i}=\Delta \mathrm{U}_{i}^{\text {vap }} / \Delta \mathrm{U}_{i}^{\text {visc }}
$$

In equation (8), $\psi_{i}$ represents the volume fraction of molecules moving into a hole left by a molecule of type $i$ in the mixture. This can be expressed as a function of molar fractions $x_{i}(i=1,2)$ and partial molar volumes $V_{i}(i=1,2)$ as follows:

$$
\begin{aligned}
& \psi_{1}=V_{1} /\left(x_{1} V_{1}+x_{2} V_{2}\right) \\
& \psi_{2}=V_{2} /\left(x_{1} V_{1}+x_{2} V_{2}\right)
\end{aligned}
$$

with the constraint of the Gibbs-Duhem relation

$$
\mathrm{x}_{1} \psi_{1}+\mathrm{x}_{2} \psi_{2}=1
$$

Substituting equation (8) to (12) into equation (1), the thermal diffusion factor is thus given by:

$$
\alpha_{\mathrm{T}}^{\mathrm{F}}=\frac{\left(\mathrm{U}_{1} / \tau_{1}-\mathrm{U}_{2} / \tau_{2}\right)}{\mathrm{x}_{1}\left(\partial \mu_{1} / \partial \mathrm{x}_{1}\right)}+\frac{\left(\mathrm{V}_{2}-\mathrm{V}_{1}\right)\left(\mathrm{x}_{1} \mathrm{U}_{1} / \tau_{1}+\mathrm{x}_{2} \mathrm{U}_{2} / \tau_{2}\right)}{\left(\mathrm{V}_{1} \mathrm{x}_{1}+\mathrm{V}_{2} \mathrm{x}_{2}\right) \mathrm{x}_{1}\left(\partial \mu_{1} / \partial \mathrm{x}_{1}\right)}
$$


The thermal diffusion factor in the Firoozabadi model is an explicit function of partial molar internal energies, volumes, the chemical potential and the ratio of the energies of vaporization and viscous flow. The value of ratio, $\tau_{i}$, is estimated to be 4.0 for most of the non-associated liquids and hydrocarbons. The Firoozabadi model is derived and explained in more detail in the next chapter.

\subsection{FUNDAMENTAL EQUATIONS IN POROUS MEDIA}

In natural porous media, such as beach sand, rye bread and wood, the distribution of pores is irregular with respect to the shape and size of the media. However, in experimental and theoretical studies, the quantities to describe the flow are usually treated in a regular manner with respect to space and time.

The standard procedure of deriving the laws governing the macroscopic variables is to start with the standard equations that the fluid obeys and then obtain the macroscopic equations by taking an average over volumes or areas containing many pores. One way to do this is through the spatial approach, where a macroscopic variable is defined as an appropriate mean over a sufficiently large representative elementary volume (REV). It is important to note that the length scale of the REV is much larger than the pore scale, but considerably smaller than the length scale of the macroscopic flow domain. This operation yields the value of the variable at the centroid of the REV. It is assumed that the result is independent of the size of the REV. A continuum model is therefore created for a porous medium based on the REV concept. Then, by introducing a Cartesian reference frame and applying the usual arguments, differential equations expressing conservation laws can be derived.

As mentioned above, the averages are not sensitive to the choice of the volume element. However, there is a distinction between the average taken with respect to a volume element of the medium, $V_{m}$, and the one taken with respect to a volume element consisting of fluid, $\mathrm{V}_{\mathfrak{f}}$, only. 
This difference lies in the definition of two velocities commonly used in porous medium. One is called the Seepage velocity, or the filtration velocity, $v_{\text {Seepage }}$, which denotes the average of the fluid velocity over the $V_{m}$. Another one is called the intrinsic average velocity, $v_{\text {intrinsic }}$, which represents the fluid velocity over a volume $V_{f}$. They are related by the Dupuit-Forchheimer relationship [2] as:

$$
\mathrm{v}_{\text {Seepage }}=\varphi \cdot \mathrm{v}_{\text {intrinsic }}
$$

where $\varphi$ is the porosity of the porous medium, which is the fraction of the total volume of the medium occupied by void space. Similarly, $1-\varphi$ is the fraction occupied by the solid.

\subsubsection{Momentum Equation}

In Darcy's law, the fluid velocity, $v$, is represented by [2]:

$$
\mathrm{v}=-\frac{\kappa}{\mu} \frac{\partial \mathrm{P}}{\partial \mathrm{x}}
$$

Here, $\frac{\partial \mathrm{P}}{\partial \mathrm{x}}$ represents the pressure gradient in the flow direction, $\mu$ is the dynamic viscosity of the fluid, and $\kappa$ represents the specific permeability or intrinsic permeability of the medium. Permeability is an important physical property of a porous medium, measuring quantitatively the ability of a porous medium to conduct fluid flow. In other words, it is the measure of the ease with which a fluid can move through a porous medium. The permeability has a dimension of an area and a unit of Darcy (d), which equals $0.987 \times 10^{-12} \mathrm{~m}^{2}$; however MUCH scientific literature uses the unit of milli-Darcy (md). In three dimensions, Eq. (15) becomes

$$
\overrightarrow{\mathbf{v}}=-\mu^{-1} \kappa \nabla \overrightarrow{\mathbf{P}}
$$


The linear Darcy's law holds for flows with low Reynolds numbers in which the driving forces are small and balanced only by the viscous forces. Yet it will break down when the flow becomes too fast and the interaction between the fluid and the pore walls becomes significant.

Considering the acceleration and other inertial effects, the Darcy's law becomes [2]:

$$
\rho_{\mathrm{f}}\left[\varphi^{-1} \frac{\partial \overrightarrow{\mathrm{v}}}{\partial \mathrm{t}}+\varphi^{-2}(\overrightarrow{\mathrm{v}} \nabla) \overrightarrow{\mathrm{v}}\right]=-\nabla \overrightarrow{\mathrm{P}}-\frac{\mu}{\kappa} \overrightarrow{\mathrm{v}}
$$

where, $\rho_{\mathrm{f}}$ is the fluid density. By assuming that the term $(\vec{v} \nabla) \vec{v}$ is zero for steady incompressible unidirectional flow, the Darcy's equation with inertial effects further becomes:

$$
\frac{\rho_{\mathrm{f}}}{\varphi} \frac{\partial \overrightarrow{\mathrm{v}}}{\partial \mathrm{t}}=-\nabla \overrightarrow{\mathrm{P}}-\frac{\mu}{\kappa} \overrightarrow{\mathrm{v}}
$$

This equation has been derived on the assumption that the partial derivative with respect to time permutes with a volume average; however, this is not always the case.

An alternative to Darcy's equation is what is commonly known as Brinkman's equation. The Brinkman's equation without the inertial terms can be expressed as follows:

$$
\nabla \overrightarrow{\mathrm{P}}=-\frac{\mu}{\kappa} \overrightarrow{\mathrm{v}}+\widetilde{\mu} \nabla^{2} \overrightarrow{\mathrm{v}}
$$

Here, the effective viscosity is represented by $\tilde{\mu} ; \mathrm{p}$ is the intrinsic fluid pressure. The Brinkman's equation is employed as a general momentum equation when the situation is more complicated; for instance, when there is a need for comparison of flow in the porous media with those in a clear fluid. It is noticed that the equation reduces to a form 
of Navier-Stokes equation as $\kappa$ approaches infinity, and to the Darcy's equation as $\kappa$ approaches zero.

\subsubsection{Conservation of Mass}

The conservation of mass can be expressed by the continuity equation as follows [2]:

$$
\varphi \frac{\partial \rho_{\mathrm{f}}}{\partial \mathrm{t}}+\nabla\left(\rho_{\mathrm{f}} \overrightarrow{\mathrm{v}}\right)=0
$$

where $\rho_{\mathrm{f}}$ is the fluid density and $\varphi$ is the porosity. $\varphi \frac{\partial \rho_{\mathrm{f}}}{\partial \mathrm{t}}$ represents the rate of increases of fluid within that volume, and the $\nabla \cdot\left(\rho_{f} \vec{v}\right)$ is the net mass flux into that volume.

When there is more than one component or species involved in a fluid flow, various components can be distinguished by utilizing a subscript $i$, which refers to the $i^{\text {th }}$ component of the mixture. Hence, the principle of mass conservation can be applied to each component in the mixture. In the absence of component generation Eq. (20) becomes:

$$
\frac{\partial \rho_{i}}{\partial t}+\nabla \cdot\left(\rho_{i} \vec{v}_{i}\right)=0
$$

where $\bar{v}_{i}$ is the intrinsic velocity of component $i$. Taking the sum of all the components, the equation becomes:

$$
\frac{\partial \rho}{\partial \mathrm{t}}+\nabla \cdot\left(\sum \rho_{\mathrm{i}} \overrightarrow{\mathrm{v}}_{\mathrm{i}}\right)=0
$$

This is analogous to the equation

$$
\frac{\partial \rho}{\partial t}+\nabla \cdot(\rho \vec{v})=0
$$


where $\vec{v}$ is the mass averaged velocity, given by:

$$
\overrightarrow{\mathrm{v}}=\frac{1}{\rho} \sum \rho_{\mathrm{i}} \overrightarrow{\mathrm{v}}_{\mathrm{i}}
$$

The motion of a component relative to the mass averaged velocity is called diffusion. Therefore, $\left(v_{i}-\vec{v}\right)$ is the diffusion velocity of component $i$, and the diffusive flux of the component i, can be represented by:

$$
\mathrm{j}_{\mathrm{i}}=\rho_{\mathrm{i}}\left(\mathrm{v}_{\mathrm{i}}-\overrightarrow{\mathrm{v}}\right)
$$

The mass conservation equation thus becomes:

$$
\frac{\partial \rho_{i}}{\partial t}+\nabla\left(\rho_{i} \vec{v}\right)=-\nabla j_{i}
$$

So far only fluid was considered. When a porous solid matrix saturated by fluid mixture is considered, the mass conservation equation can be obtained by multiplying Eq. (26) by $\varphi$ as follows:

$$
\varphi \frac{\partial \rho}{\partial \mathrm{t}}+\varphi(\nabla \rho \mathrm{v})=\varphi \nabla j
$$

The diffusive mass flux $(j \varphi)$ in the porous medium is the rate of flow of mass across unit cross-sectional area of the medium.

In instances when there is a direct coupling between heat and mass transport, in other words, the Soret and Dufour effects are not negligible, [2] the mass conservation equation appears as follows for isobaric conditions: 


$$
\varphi \frac{\partial \rho}{\partial \mathrm{t}}+\varphi(\nabla \rho \overrightarrow{\mathrm{v}})=\varphi \nabla\left(\mathrm{D}^{\mathrm{M}} \nabla \overrightarrow{\mathrm{C}}+\mathrm{D}^{\mathrm{T}} \nabla \overrightarrow{\mathrm{T}}\right)
$$

where $D^{M}$ is the mass diffusion coefficient of the porous medium, $D^{T}$ is the thermal diffusion coefficient of the porous medium, and $\mathrm{C}$ is the concentration. The Soret coefficient can be calculated as follows:

$$
\mathrm{S}_{\mathrm{T}}=\frac{\mathrm{D}^{\mathrm{T}}}{\mathrm{D}^{\mathrm{M}}}
$$

The Soret effect refers to mass flux produced by a temperature gradient, and the Dufour effect refers to heat flux produced by a concentration gradient. When the isobaric condition does not exist the mass conservation equation becomes:

$$
\varphi \frac{\partial \rho}{\partial \mathrm{t}}+\varphi(\nabla \rho \overrightarrow{\mathrm{v}})=\varphi \nabla\left(\mathrm{D}^{\mathrm{M}} \nabla \overrightarrow{\mathrm{C}}+\mathrm{D}^{\mathrm{T}} \nabla \overrightarrow{\mathrm{T}}+\mathrm{D}^{\mathrm{P}} \nabla \overrightarrow{\mathrm{P}}\right)
$$

where $\mathrm{D}^{\mathrm{P}}$ is the pressure diffusion coefficient. Therefore, the diffusive mass flux in the porous medium can be generally expressed as:

$$
\vec{j}=-\left(D^{M} \nabla \vec{C}+D^{T} \nabla \vec{T}+D^{P} \nabla \vec{P}\right)
$$

\subsubsection{Energy Equation}

A simple situation is considered first in which the medium is isotropic and the radiative effects, viscous dissipation and the work done by pressure changes are assumed negligible. By taking averages over an elemental volume of the medium, the equations for solid and liquid phases are as follows:

$$
(1-\varphi)(\rho c)_{s} \frac{\partial T_{s}}{\partial t}=(1-\varphi) \nabla\left(k_{s} \nabla \vec{T}_{s}\right)+(1-\varphi) q_{s}^{\prime \prime}
$$




$$
\varphi(\rho c)_{f} \frac{\partial T_{f}}{\partial t}+\left(\rho c_{p}\right)_{f} \vec{v} \nabla T_{f}=\varphi \nabla \cdot\left(k_{f} \nabla \vec{T}_{f}\right)+\varphi q_{f}^{\prime \prime \prime}
$$

The subscripts $\mathrm{s}$ and $\mathrm{f}$ refer to the solid and fluid phases, respectively. $\mathrm{c}$ is the specific heat of the solid, $c_{p}$ is the specific heat of the fluid at constant pressure, $\mathrm{k}$ is the thermal conductivity, $\mathrm{q}^{m}$ is the heat production per unit volume, and $T_{s}$ and $T_{f}$ are the temperatures of the solid and fluid phases.

The above equations assume that the surface porosity is equal to the porosity of the medium. This is important for the conduction term, $(1-\varphi) \nabla\left(\mathrm{k}_{\mathrm{s}} \nabla \mathrm{T}_{\mathrm{s}}\right)$, in Eq. (32), which represents the net rate of heat conduction into a unit volume of the solid. In Eq. (33), there is a convective term, $\left(\rho c_{p}\right)_{f} \vec{v} \nabla T_{f}$, which represents the rate of change of thermal energy per unit volume of fluid due to convection.

Now, by setting $T_{s}$ equals to $T_{f}$, and calling it the temperature of the mixture, $T$, and then adding Eq. (32) and (33), the overall energy equation can be obtained, as well as the overall heat capacity per unit volume, overall thermal conductivity, and overall heat production per unit volume of the medium can be obtained.

$$
(\rho c)_{m} \frac{\partial T}{\partial t}+(\rho c)_{f} v \nabla \vec{T}=\nabla \cdot\left(k_{m} \nabla \vec{T}\right)_{+} q_{m}^{\prime \prime \prime}
$$

where,

$$
\begin{aligned}
& (\rho c)_{m}=(1-\varphi)(\rho c)_{s}+\varphi\left(\rho c_{p}\right)_{f} \\
& k_{m}=(1-\varphi) k_{s}+\varphi k_{f} \\
& q_{m}^{\prime \prime \prime}=(1-\varphi) q_{s}^{\prime \prime \prime}+\varphi q_{f}^{\prime \prime \prime}
\end{aligned}
$$




\subsubsection{Boussinesq Approximation}

An equation of state (EOS) is required to complement the equations of momentum, mass and energy. There are several cases where EOS can be simplified as a single expression. For example, when the fluid density changes only with temperature, the EOS can be represented as:

$$
\rho_{\mathrm{f}}=\rho_{0}\left[1-\beta_{\mathrm{T}}\left(\mathrm{T}-\mathrm{T}_{0}\right)\right]
$$

where, $\rho_{0}$ is the fluid density at a reference temperature $T_{0}$, and $\beta_{T}$ is the coefficient of thermal expansion due to the temperature change.

When there are small isobaric changes in the temperature and concentration, the mixture density $\rho$ depends linearly on both $\mathrm{T}$ and $\mathrm{C}$, which is the case of double-diffusive convection.

$$
\rho=\rho_{0}\left[1-\beta_{T}\left(T-T_{0}\right)-\beta_{C}\left(C-C_{0}\right)\right]
$$

the subscript 0 refers to a reference state, and $\beta_{\mathrm{T}}$ can be represented as :

$$
\beta_{\mathrm{T}}=-(1 / \rho)(\partial \rho / \partial \mathrm{T})_{\mathrm{p}, \mathrm{C}}
$$

$\beta_{\mathrm{C}}$ is the volumetric concentration expansion coefficient, given by :

$$
\beta_{\mathrm{C}}=-(1 / \rho)(\partial \rho / \partial \mathrm{C})_{T, \mathrm{p}}
$$

When conditions are non-isobaric small changes in the temperature, concentration, and pressure will all have an effect on the mixture's density $\rho$ : 


$$
\rho=\rho_{0}\left[1-\beta_{T}\left(T-T_{0}\right)-\beta_{C}\left(C-C_{0}\right)-\beta_{p}\left(P-P_{0}\right)\right]
$$

where $\beta_{p}$ is the compressibility coefficient, given by :

$$
\beta_{\mathrm{p}}=-(1 / \rho)(\partial \rho / \partial \mathrm{p})_{\mathrm{T}, \mathrm{C}}
$$

the coefficients in Eq. (37), (38) and (40) are evaluated at the reference state.

For more complicated cases, cubic EOS may be used to calculate thermodynamic properties including the density. Cubic EOS such as the Ping-Robinson (PR) EOS and the Cubic-Plus-Association (CPA) EOS are widely used in simulations of oil reservoirs. The CPA-EOS may be used to determine the density and other equilibrium properties for polar mixtures such as water-methanol and water-ethanol. It has been demonstrated that PR-EOS can provide great accuracy for light and heavy hydrocarbons. In the next chapter the PR-EOS is derived and explained in detail. 


\section{CHAPTER 3: THEORETICAL ANALYSIS AND COMPARISON WITH EXPERIMENTAL DATA FOR THE MOLECULAR AND THERMAL DIFFUSION COEFFICIENTS FOR A TERNARY MIXTURE}

\subsection{INTRODUCTION}

Firoozabadi's model is based on the thermodynamics theory of an irreversible process. It develops analytical relations between the thermal, molecular and pressure diffusion coefficients, and the fluid properties including temperature, pressure, density, fugacity, and viscosity. Unlike other models, the Firoozabadi's model is not limited to the use of binary mixtures, but can be extended to multicomponent mixtures.

Firoozabadi's model has been widely applied in hydrocarbon mixtures, as well as polar mixtures such as water-ethanol and water-methanol. In doing so, it has shown success in predicting the thermal and molecular diffusion coefficients. For the case of hydrocarbon mixtures, the properties of the fluid mixture at equilibrium states are evaluated using the Peng-Robinson (PR) equation of state. For polar mixtures, the Cubic Plus Association (CPA) equation of state is used.

Faruque et al. [39] compared the results obtained using the Firoozabadi model, with the PR equation of state, to the experimental data published by Rutherford and Roof [24]. A Methane and $n$-Butane fluid system at a composition of 0.4-mole fraction of methane and 0.6-mole fraction of normal-butane was used. The pressure ranged between 9 and $21 \mathrm{Mpa}$ and the temperature ranged between 320 and $377 \mathrm{~K}$. The comparison revealed a positive agreement with the experimental results at higher pressure. However, at low pressure, there existed a discrepancy between the numerical and the experimental results. Nevertheless, the deviation between the numerical calculations and the experimental results for the range of pressures and temperatures did not exceed $15 \%$.

Saghir et al. [38] compared the results obtained using the Firoozabadi model, with the CPA equation of state, to the experimental data obtained by Platten [40]. Two polar 
mixtures, methanol-water and ethanol-water, with 0.835 mole fraction of methanol and 0.779 mole fraction of ethanol respectively were utilized. An atmospheric pressure was maintained with a temperature of $310.65 \mathrm{~K}$. The comparison revealed a close agreement between the numerical and experimental data. In addition, Saghir et al. compared results obtained by the Firoozabadi model with the results obtained by the Haase and Kempers model. This comparison indicated that the Firoozabadi model was more accurate and provided the best estimation for both polar mixtures. Compared to other models, it is very reliable for binary mixtures and also shows significant improvement in the prediction of the thermal diffusion process.

Although much attention has been focused on obtaining the thermal diffusion coefficient for different binary liquid mixtures, the results available in the literature differ considerably. Therefore, research groups from various universities gathered at Fontainebleau, France, and started a ground-based measurement campaign with the aim of establishing a reliable base of the thermal diffusion and molecular diffusion coefficients for three binary mixtures of n-Dodecane $\left(\mathrm{C}_{12} \mathrm{H}_{26}\right)$, Isobutylbenezene (IBB), and 1, 2, 3, 4-Tetrahydronaphthalene (THN). These three binary mixtures were at a fixed concentration of $50 \mathrm{wt} \%$ and at a mean temperature of $25^{\circ} \mathrm{C}$. Furthermore, to the best of the author's knowledge, there were no reported measured thermal diffusion coefficients for ternary hydrocarbon mixtures until Platten et al. [9] reported an experimental study measuring the thermal diffusion coefficient for a ternary mixture of $\mathrm{C}_{12} \mathrm{H}_{26}$-THN-IBB at a concentration of $33 \mathrm{wt} \%$ and a mean temperature of $25^{\circ} \mathrm{C}$.

In this study, attempts are made to calculate the density and molecular and thermal diffusion coefficients of three binary mixtures formed with $\mathrm{C}_{12} \mathrm{H}_{26}$, THN, and IBB using Firoozabadi's model. A comparison is then made with the Fontainebleau benchmark values. The main objective is to calculate the thermal diffusion coefficients for the ternary mixture of $\mathrm{C}_{12} \mathrm{H}_{26}$-THN-IBB using Firoozabadi model and compare them with the experimental data, which to the best of the author's knowledge has never been accomplished before. In addition, the study then attempts to correlate the thermal 
diffusion coefficients in the ternary mixture with those in the three binary mixtures suggested by Platten et al. [9].

\subsection{THEORETICAL DERIVATION OF THE FIROOZABADI MODEL}

The mass, momentum, and energy balance equations are the general mathematical expressions for all physical conservation laws. They form the basis for thermal diffusion theory. The general conservation equation for any quantity $\psi_{k}$ of a component $\mathrm{k}$ inside a mixture fluid with the density $\rho_{k}$, velocity $\overrightarrow{v_{k}}$, efflux $J_{k}$, and body source $\phi_{k}$ is written as follows [11]:

$$
\frac{\partial \rho_{k} \psi_{k}}{\partial t}+\nabla \cdot\left(\overrightarrow{v_{k}} \rho_{k} \psi_{k}\right)=-\nabla \cdot J_{k}+\rho_{k} \phi_{k}
$$

The first term of Eq. (41) on the left hand side is the time rate of change of the quantity $\psi_{\mathrm{k}}$ per unit volume, and the second term is the rate of convection per unit volume. On the right hand side, the first term represents the surface flux source, and the second term is the volume source.

The following transformation of a substantial derivative is useful in the derivation of conservation equations of mass, momentum, and energy,

$$
\rho \frac{\mathrm{Da}}{\mathrm{Dt}}=\frac{\partial \rho \mathrm{a}}{\partial \mathrm{t}}+\nabla \cdot(\rho \mathrm{a} \overrightarrow{\mathrm{v}})
$$

where, a represents an arbitrary local property, which can be a scalar, or a vector, or a tensor. The averaged or the Barycentric velocity is represented by $\vec{v}$, and can be expressed as follows: 


$$
\vec{v}=\sum_{k=1}^{n} \frac{\rho_{k} \overrightarrow{v_{k}}}{\rho}
$$

where, $\rho_{k}$ is the density of component $k$ in the multicomponent mixture, $\vec{v}_{k}$ is the velocity of component $\mathrm{k}$, and $\rho$ is the density of the mixture.

By applying the basic conservation law of mass, momentum, and energy, the following equations can be obtained:

$$
\begin{gathered}
\frac{D \rho}{D t}=-\rho \nabla \cdot \vec{v} \\
\rho \frac{D c_{k}}{D t}=-\nabla \cdot J_{k}+\sum_{j=1}^{r} v_{k j} R_{j} \\
\rho \frac{D u}{D t}=-\nabla \cdot J_{q}-p \nabla \cdot \vec{v}-\pi: \nabla \vec{v}+\sum_{k=1}^{n} J_{k} \cdot \overrightarrow{F_{k}}
\end{gathered}
$$

where,

$c_{k}$ is the component fraction, $c_{k}=\frac{\rho_{k}}{\rho}$ and $\sum_{k=1}^{n} c_{k}=1$;

$J_{k}$ is the diffusion flux of component $k, J_{k}=\rho_{k}\left(\overrightarrow{v_{k}}-\vec{v}\right)$ and $\sum_{k=1}^{n} J_{k}=0$;

$R_{j}$ is the chemical reaction rate of component $j$;

$\mathrm{v}_{\mathrm{kj}} \mathrm{R}_{\mathrm{j}}$ is the production rate of $\mathrm{k}$ component per unit volume in the $\mathrm{j}$-th chemical reaction, such that $\sum_{k=1}^{n} v_{k j}=0$ for $j=1,2, \ldots, r$; 
$u$ is the internal energy of the fluid;

$\mathrm{J}_{\mathfrak{q}}$ is the total heat flux;

$\mathrm{p}$ is the scalar pressure;

$\pi$ is the viscous pressure tensor; and

$F_{k}$ is the field force acting on component $k$.

Based on the law of non-equilibrium thermodynamics theory, the small elements inside a system can be obtained using the following entropy equation:

$$
\mathrm{T} \frac{\mathrm{Ds}}{\mathrm{Dt}}=\frac{\mathrm{Du}}{\mathrm{Dt}}+\mathrm{p} \frac{\mathrm{Dv}}{\mathrm{Dt}}-\sum_{\mathrm{i}=1}^{\mathrm{n}} \mu_{\mathrm{i}} \frac{\mathrm{Dc}}{\mathrm{Dt}}
$$

Therefore, the rate of entropy production, $\sigma=\frac{\mathrm{Ds}}{\mathrm{Dt}}$, can be written as follows:

$$
\sigma=-\frac{1}{\mathrm{~T}^{2}}\left[\mathrm{~J}_{\mathrm{q}}-\sum_{\mathrm{k}=1}^{\mathrm{n}} \frac{\mathrm{H}_{\mathrm{k}}}{\mathrm{M}_{\mathrm{k}}} \mathrm{J}_{\mathrm{k}}\right] \nabla \mathrm{T}-\frac{1}{\mathrm{~T}} \sum_{\mathrm{k}=1}^{\mathrm{n}} \mathrm{J}_{\mathrm{k}}\left\{\frac{\nabla_{\mathrm{T}} \mu_{\mathrm{k}}}{\mathrm{M}_{\mathrm{k}}}-\mathrm{F}_{\mathrm{k}}\right\}-\frac{1}{\mathrm{~T}} \pi: \nabla \overrightarrow{\mathrm{v}}-\frac{1}{\mathrm{~T}} \sum_{\mathrm{j}=1}^{\mathrm{T}} \mathrm{R}_{\mathrm{j}} \mathrm{A}_{\mathrm{j}}
$$

where:

$M_{k}$ is the molecular weight of the $k$-th component;

$\mathrm{n}$ is the total number of components;

$r$ is the total number of chemical reactions; and

$A_{j}$ is the chemical affinities of the reactions $j(j=1,2, \ldots, r)$, defined as follows: 


$$
A_{j}=\sum_{k=1}^{n} v_{k j} \mu_{k}
$$

where, $v_{k j} \mu_{k}$ is the chemical production rate of the $k$-th component due to the $j$-th reaction.

From Eq. (48), it is evident that there are four distinct thermodynamic driving forces, the gradient of temperature, the gradient of chemical potential and external force, the gradient of velocity, and the chemical reaction rate. Nevertheless, these four forces are in three different tensorial orders. The first two forces belong to the same order 1 , but the third force is of order 2 , and the fourth is a scalar or an order 0 .

Based on the assumptions known as the Curie assumptions, there are four postulates that can be made,

1). The quasi-equilibrium postulate states that the equilibrium thermodynamics relations apply to systems that are not in equilibrium, provided that the gradients are not too large.

2). The linear postulate states that all the fluxes in the system may be written as linear relations involving all the forces.

3). The Curie postulate states that no coupling of fluxes and forces occur if the difference in tensorial orders of the flux and force is an odd number.

4). The Onsager reciprocal relations postulate states that, in the absence of a magnetic field the matrix of the coefficients in the flux-force relations is symmetric.

Therefore, for simplicity the following relationship can be used:

$$
\mathrm{J}_{\mathrm{q}}^{\prime}=\mathrm{J}_{\mathrm{q}}-\sum_{\mathrm{k}=1}^{\mathrm{n}} \frac{\mathrm{H}_{\mathrm{k}}}{\mathrm{M}_{\mathrm{k}}} \mathrm{J}_{\mathrm{k}}
$$


Based on the Curie assumptions, each of the $J_{k}$ must depend linearly on all of the $\vec{\nabla}_{T} \vec{\mu}_{k}$ as well as on $\nabla \vec{T}$, and $J_{q}^{\prime}$ must depend linearly on $\nabla \vec{T}$ as well as the $\vec{\nabla}_{T} \vec{\mu}_{k}$, however, neither $J_{k}$ nor $J_{q}^{\prime}$ can depend on $\nabla \cdot \vec{v}$ or $R_{k}$. Similarly the viscous pressure tensor $\pi$ will depend on the tensor $\nabla \cdot \vec{v}$, and also on the scalar driving forces $R_{k}$ multiplied by the unit tensor.

The "coupling" between $\pi$ and the chemical reactions have not been studied, and it is not of interest to thermal diffusion research. The entropy generation rate in thermal diffusion research is simplified as:

$$
\sigma=-\frac{1}{T^{2}} J_{q}^{\prime} \cdot \nabla \vec{T}-\frac{1}{T} \sum_{k=1}^{n-1} J_{k} \cdot\left\{\frac{\nabla_{T} \mu_{k}}{M_{k}}-\frac{\nabla_{T} \mu_{n}}{M_{n}}\right\}
$$

The thermodynamic force conjugate to $J_{q}^{\prime}$ is the temperature gradient. The thermodynamic force conjugate to $\mathrm{J}_{\mathrm{k}}$ is the chemical potential gradient under constant temperature $\mathrm{T}$, and external force, not containing a term of temperature gradient. It is important to note that $\mathrm{J}_{q}^{\prime}$ is the flux conjugating to the temperature gradient, not $\mathrm{J}_{\mathrm{q}}$. From Eq. (50), it is clear that the difference between $J_{q}$ and $J_{q}^{\prime}$ represents a transfer of heat due to mass diffusion. Therefore, the quantity $\mathrm{J}_{\mathrm{q}}^{\prime}$ also represents an irreversible heat flow.

If only the gravitational external force exists, the term $F_{k}$ is eliminated because the total summation of $\mathrm{J}_{\mathrm{k}}$ is zero.

Based on the assumptions of the irreversible process theory, there exist linear relations between the two fluxes, and two driving forces, which are called Onsager Equations. Onsager [32] Equations are given as follows.

$$
\mathrm{J}_{\mathrm{q}}^{\prime}=-\mathrm{L}_{\mathrm{qq}} \frac{1}{\mathrm{~T}^{2}} \nabla \overrightarrow{\mathrm{T}}-\frac{1}{\mathrm{~T}} \sum_{\mathrm{k}=1}^{\mathrm{n}-1} \mathrm{~L}_{\mathrm{qk}}\left\{\frac{\nabla_{\mathrm{T}} \bar{\mu}_{\mathrm{k}}}{\mathrm{M}_{\mathrm{k}}}-\frac{\nabla_{\mathrm{T}} \bar{\mu}_{\mathrm{n}}}{\mathrm{M}_{\mathrm{n}}}\right\}
$$




$$
\mathrm{J}_{\mathrm{i}}=-\mathrm{L}_{\mathrm{iq}} \frac{1}{\mathrm{~T}^{2}} \nabla \mathrm{T}-\frac{1}{\mathrm{~T}} \sum_{\mathrm{k}=1}^{\mathrm{n}-1} \mathrm{~L}_{\mathrm{ik}}\left\{\frac{\nabla_{\mathrm{T}} \mu_{\mathrm{k}}}{\mathrm{M}_{\mathrm{k}}}-\frac{\nabla_{\mathrm{T}} \mu_{\mathrm{n}}}{\mathrm{M}_{\mathrm{n}}}\right\}
$$

where $\mathrm{L}_{\mathrm{qq}}, \mathrm{L}_{\mathrm{qk}}, \mathrm{L}_{\mathrm{iq}}, \mathrm{L}_{\mathrm{ik}}$ are the Onsager corresponding phenomenological coefficients.

A symmetric relation exists in the above equations, which is one of the assumptions made in the irreversible process theory.

Knowing the Gibbs-Duhem expression [11], the relation between chemical potential and fugacity and the relation between chemical potential and the partial molar volume, one may modify Eq. (53) to take the following form:

$$
J_{i}=-\frac{R L_{i i}}{M_{i} x_{i} M_{n} x_{n}}\left\{\begin{array}{l}
\left.\frac{M_{i} x_{i}}{L_{i i}} \sum_{k=1}^{n-1} L_{i k} \sum_{j=1}^{n-1} \frac{M_{j} x_{j}+M_{n} x_{n} \delta_{j k}}{M_{j}} \sum_{i=1}^{n-1} \frac{\partial \ln f_{j}}{\partial x_{1}}\right|_{x_{1}, T, P} \nabla x_{1} \\
\frac{M_{i} x_{i}}{R T L_{i i}} \sum_{k=1}^{n-1} L_{i k}\left[\sum_{j=1}^{n-1} x_{j} \overline{v_{j}}+\frac{M_{n} x_{x}}{M_{k}} \overline{v_{k}}-\frac{1}{\rho_{m}}\right] \nabla P \\
+\frac{M_{i} x_{i} M_{n} x_{n} L_{i q}}{R L_{i i}} \frac{\nabla T}{T^{2}}
\end{array}\right\}
$$

where: $R$ is gas constant, $x_{i}$ is the mole fraction of component $i, f_{i}$ is the fugacity of component $i, \bar{v}_{i}$ is the molar volume of component $i, \rho_{m}$ is the molar density of the mixture, $\mathrm{P}$ is the pressure, and $\delta_{\mathrm{ik}}$ is the delta function, $\delta_{\mathrm{ik}}=1$, when $\mathrm{i}=\mathrm{k}$, and $\delta_{\mathrm{ik}}=0$ when $i \neq k$.

The mass fluxes can be written in a short vector form,

$$
\overrightarrow{\mathrm{J}}=-\rho_{\mathrm{m}}\left(\widetilde{\mathrm{D}}^{\mathrm{M}} \overrightarrow{\nabla \mathrm{X}}+\widetilde{\mathrm{D}}^{\mathrm{T}} \overrightarrow{\nabla \mathrm{T}}+\widetilde{\mathrm{D}}^{\mathrm{P}} \overrightarrow{\nabla \mathrm{P}}\right)
$$


where: $\widetilde{\mathrm{D}}^{\mathrm{M}}$ is the molecular diffusion coefficient tensor; $\widetilde{\mathrm{D}}^{\mathrm{T}}$ is thermal diffusion coefficient tensor; $\widetilde{\mathrm{D}}^{\mathrm{P}}$ is the pressure diffusion coefficient tensor; $\overrightarrow{\nabla \mathrm{X}}$ is the tensor of all mole fraction gradient; $\overrightarrow{\nabla \mathrm{P}}$ is the vector of the pressure gradient; $\overrightarrow{\nabla \mathrm{T}}$ is the vector of all temperature gradients; $\vec{J}$ is the tensor of all component mass fluxes, $J_{i} ; \rho_{m}$ is the molar density of the mixture.

By comparing Eq. (54) and Eq. (55), the three diffusion coefficients become:

$$
\left.\begin{array}{l}
\widetilde{D}_{i j}^{M}=\left.\frac{R}{\rho_{m} x_{x} M_{n}} \sum_{k=1}^{n-1} L_{i k} \sum_{j=1}^{n-1} \frac{x_{j} M_{j}+x_{n} M_{n} \delta_{j k}}{M_{j}} \frac{\partial \ln f_{j}}{\partial x_{1}}\right|_{x_{1}, T, P} \\
\widetilde{D}_{i}^{T}=\frac{L_{i q}}{\rho_{m} T^{2}} \\
\widetilde{D}_{i}^{P}=\frac{1}{\rho_{m} T x_{n} M_{n}} \sum_{k=1}^{n-1} L_{i k}\left[\sum_{j=1}^{n-1} x_{j} \overline{V_{j}}+\frac{x_{n} M_{n}}{M_{k}} \overline{V_{k}}-\frac{1}{\rho_{m}}\right]
\end{array}\right\}
$$

Therefore, for a binary system without pressure diffusion present, the mass flux of one component can be represented as:

$$
\overrightarrow{\mathrm{J}_{1}}=-\rho_{\mathrm{m}}\left(\widetilde{\mathrm{D}}^{\mathrm{M}} \nabla \overrightarrow{\mathrm{x}}_{1}+\widetilde{\mathrm{D}}^{\mathrm{T}} \nabla \overrightarrow{\mathrm{T}}\right)
$$

For ternary systems without pressure diffusion present, there are two independent mass fluxes and can be represented as:

$$
\begin{aligned}
& \overrightarrow{J_{1}}=-\rho_{m}\left(\widetilde{D}_{11}^{M} \nabla x_{1}+\widetilde{D}_{12}^{M} \nabla x_{2}+\widetilde{D}_{1}^{T} \nabla T\right) \\
& \overrightarrow{J_{2}}=-\rho_{m}\left(\widetilde{D}_{21}^{M} \nabla x_{1}+\widetilde{D}_{22}^{M} \nabla x_{2}+\widetilde{D}_{2}^{T} \nabla T\right)
\end{aligned}
$$


The pressure diffusion is usually ignored in experimental approaches because the magnitude of pressure diffusion contribution is about three orders smaller than thermal diffusion. The Firoozabadi model gives the formula for the estimation of thermal and molecular diffusion coefficients. However, the calculation depends on all of the thermodynamic parameters, such as density, enthalpy, internal energy and fugacity. Therefore, a good equation of state, such as Peng-Robinson, is crucial for the accuracy of the estimation.

Therefore, all three diffusion coefficients are defined as functions of the thermodynamic properties of the mixture and Onsager coefficients. Since the mass flux for a uniform temperature distribution is well developed, the Onsager coefficients, $\mathrm{L}_{\mathrm{ij}}$, can be determined. The Onsager coefficients, $\mathrm{L}_{\mathrm{iq}}$, which represents the Soret effect, have been the target of all thermal diffusion models; however, the Firoozabadi model is the only model that can accurately predict these coefficients not only for multicomponent mixtures but also for non-ideal multicomponent mixtures. The following derivation illustrates the Firoozabadi approach.

The heat flux, $J_{q}$, is based on irreversible thermodynamics and molecular kinetic theory involving the heat of transport and can be given as:

$$
J_{\mathrm{q}}=\sum_{\mathrm{k}=1}^{\mathrm{n}} \frac{\mathrm{Q}_{\mathrm{k}}}{\mathrm{M}_{\mathrm{k}}} \mathrm{J}_{\mathrm{k}}
$$

where, $\mathrm{Q}_{\mathrm{k}}$ is the heat of transport of component $\mathrm{k}$.

Therefore,

$$
J_{q}^{\prime}=J_{q}-\sum_{k}^{n} \frac{H_{k}}{M_{k}} J_{k}=\sum_{k=1}^{n} \frac{Q_{k}-H_{k}}{M_{k}} J_{k}=\sum_{k}^{n} \frac{Q_{k}^{*}}{M_{k}} J_{k}
$$

where, $\mathrm{Q}_{\mathrm{k}}^{*}$ is the net heat of transport of component $\mathrm{k}$. 
Substituting Eq. (61) into the entropy production Eq. (51), and applying the irreversible process theory, the following expression is obtained:

$$
\mathrm{J}_{\mathrm{i}}=-\sum_{\mathrm{k}=1}^{\mathrm{n}-1} \mathrm{~L}_{\mathrm{ik}}\left(\frac{\mathrm{Q}_{\mathrm{k}}^{*}}{\mathrm{M}_{\mathrm{k}}}-\frac{\mathrm{Q}_{\mathrm{n}}^{*}}{\mathrm{M}_{\mathrm{n}}}\right) \frac{1}{\mathrm{~T}^{2}} \nabla \mathrm{T}-\frac{1}{\mathrm{~T}} \sum_{\mathrm{k}=1}^{\mathrm{n}-1} \mathrm{~L}_{\mathrm{ik}}\left\{\frac{\nabla_{\mathrm{T}} \mu_{\mathrm{k}}}{\mathrm{M}_{\mathrm{k}}}-\frac{\nabla_{\mathrm{T}} \mu_{\mathrm{n}}}{\mathrm{M}_{\mathrm{n}}}\right\}
$$

Comparing Eq. (62) with Eq. (53), it is quite evident that

$$
L_{i q}=\sum_{k=1}^{n-1} L_{i k}\left(\frac{Q_{k}^{*}}{M_{k}}-\frac{Q_{n}^{*}}{M_{n}}\right)
$$

As previously mentioned, the Firoozabadi modelled the net heat transport for component $i$ in the mixture as:

$$
Q_{i}^{*}=-\frac{\Delta \overline{U_{i}}}{\tau_{i}}+\left(\sum_{j=1}^{n} \frac{x_{j} \Delta \overline{U_{j}}}{\tau_{j}}\right) \frac{V_{i}}{\sum_{j=1}^{n} x_{j} V_{j}}
$$

Therefore, the Firoozabadi model is completely established theoretically.

\subsection{MASS TRANSFER IN MULTICOMPONENT MIXTURE}

For an ideal gas, the molecular diffusion coefficient can be obtained by a theoretical approach. However, for non-ideal mixtures, empirical expressions are usually used. For binary hydrocarbon mixtures, the following expression given by Hayduk-Minhas in 1982 (see reference Taylor and Krishna, 1993) has been shown to be the best,

$$
D_{i j}^{o}=13.3 \times 10^{-8} V_{i}^{-0.71} \mu_{j}^{\left(10.2 / V_{1}-0.791\right)} T^{1.47}
$$


where, $D_{i j}^{o}$ is the molecular diffusion coefficient of $\mathrm{i}$-th component infinitely diluted in a binary mixture, $V_{i}$ is the specific molar volume of component $i$, and $\mu_{j}$ is the viscosity of $\mathrm{j}$-th component, the molecular diffusion coefficient is calculated based on the binary coefficients. The expression used here is based on Taylor and Krishna's approach,

$$
D_{i j}^{o}=\left(D_{i j}^{o}\right)^{x_{i}}\left(D_{j i}^{o}\right)^{x_{j}} \prod_{k=1, k \neq i, j}^{n}\left(D_{j k}^{o} D_{j k}^{o}\right)^{x_{k} / 2}
$$

where, $D_{i j}^{o}$ is the molecular diffusion coefficient of -th component in the multicomponent mixture.

Based on the multi-component mass transfer theory, the mass flux is given by:

$$
\overline{J_{m}}=-\rho_{m} B^{-1} \Gamma \nabla X
$$

where, $\mathrm{B}$ and $\Gamma$ are matrices, the elements of which are defined by the following formulae:

$$
\begin{array}{ll}
B_{i j}=-x_{i}\left(\frac{1}{D_{i j}^{o}}-\frac{1}{D_{i n}^{o}}\right), & i \neq j \\
B_{i j}=\sum_{k=1, k \neq i}^{n} \frac{x_{k}}{D_{i k}^{o}}+\frac{x_{i}}{D_{i n}^{o}}, \quad i=j
\end{array}
$$

and

$$
\Gamma_{i j}=x_{i} \frac{1}{f_{i}} \frac{\partial f_{i}}{\partial x_{j}}
$$

From Fick's law, the mass flux can be written as:

$$
\overline{J_{m}}=-\rho_{m} D^{M} \nabla X
$$


Comparing Eq. (69) with Eq. (66), the following is obtained:

$$
\mathrm{D}^{\mathrm{M}}=\mathrm{B}^{-1} \Gamma
$$

Therefore, the diffusion coefficient $\mathrm{D}_{\mathrm{ij}}^{\mathrm{M}}$ can be determined based on all the elements in $\mathrm{B}$ and $\Gamma$ matrices.

\subsection{EQUATION OF STATE: PENG-ROBINSON (PR)}

The Peng-Robinson (PR) equation of state is given in the form:

$$
\left[P+\frac{a(T)}{v(v+b)+b(v-b)}\right](v-b)=R T
$$

where, $v$ is the molar volume; $a$ is the attraction parameter; $b$ is the hard-core parameter or co-volume parameter.

Both $\mathrm{a}$ and $\mathrm{b}$ are given as functions of temperature and pressure at the critical point, that is:

$$
\left.\begin{array}{l}
\mathrm{a}\left(\mathrm{T}_{\mathrm{c}}\right)=0.45724 \frac{\mathrm{R}^{2} \mathrm{~T}_{\mathrm{c}}^{2}}{\mathrm{P}_{\mathrm{c}}} \\
\mathrm{b}=0.07780 \frac{\mathrm{RT}_{\mathrm{c}}}{\mathrm{P}_{\mathrm{c}}}
\end{array}\right\}
$$

where, $T_{c}$ is the critical temperature; $P_{c}$ is the critical pressure.

The compressibility factor, $\mathrm{z}$, can be defined as:

$$
\mathrm{Pv}=\mathrm{zRT}
$$


Based on the Peng-Robinson equation, the compressibility factor, $z$, can be given by the following equation:

$$
z^{3}-(1-B) z^{2}+\left(A-3 B^{2}-2 B\right) z-\left(A B-B^{2}-B^{3}\right)=0
$$

where,

$$
\left.\begin{array}{l}
A(T)=\frac{a(T) P}{R^{2} T^{2}} \\
B=\frac{b P}{R T}
\end{array}\right\}
$$

For mixtures, the parameters $\mathrm{a}$ and $\mathrm{b}$ are defined according to certain mixing rules. The following mixing rules for hydrocarbon mixtures are commonly used:

$$
\left.\begin{array}{l}
a=\sum_{i=1}^{n} \sum_{j=1}^{n} x_{i} x_{j} a_{i j} \\
a_{i j}=\left(1-\varepsilon_{i j}\right) a_{i}^{1 / 2} a_{j}^{1 / 2} \\
b=\sum_{i=1}^{n} x_{i} b_{i}
\end{array}\right\}
$$

where, $a_{i}$ and $b_{i}$ are the parameters for pure components; $\varepsilon_{i j}$ is the interaction parameter between the i-th and $\mathrm{j}$-th components.

The fugacity of component $i$ in the mixture can be calculated using the equation:

$$
\ln \left(\frac{f_{i}}{x_{i} P}\right)=\frac{b_{i}}{b}(z-1)+\ln (z-B)-\frac{A}{2 \sqrt{2} B}\left(\frac{2.0}{a} \sum_{j=1}^{n} x_{i} a_{i j}-\frac{b_{i}}{b}\right) \ln \left(\frac{z+(1+\sqrt{2}) B}{z+(1-\sqrt{2}) B}\right)
$$




\subsection{THERMAL AND MOLECULAR DIFFUSION: EXPERIMENTAL APPROACH}

The experiments for both binary and ternary systems were performed in a thermogravitational column. The details are provided by Dutrieux et al. [41], however, a brief description is given in this section. In the experiments, two copper plates were maintained at constant temperature by a water flow directly pumped from a thermostated bath. Spacers imposed the distance between the two copper plates. The top spacer had two holes to allow filling of the column and also for air to escape during the filling. The thermogravitational column had a height $\left(\mathrm{L}_{z}\right)$ of $53 \mathrm{~cm}$, a width $\left(\mathrm{L}_{\mathrm{x}}\right)$ of $1.59 \mathrm{~mm}$, and depth $\left(L_{y}\right)$ of $3.0 \mathrm{~cm}$. Along the thermogravitational column, there were five sampling taps. For a binary system, the removed samples were analyzed by measuring the density with a quartz vibrating U-tube densimeter, with an accuracy of $\pm 2 \times 10^{-6} \mathrm{~g} / \mathrm{cm}^{3}$. In the case of a ternary system, at each tap location, two quantities were measured, namely, the density and the index of refraction. The Belllingham-Stanley refractometer RFM 340 was used to measure the index of refraction and had an accuracy of $\pm 4 \times 10^{-5}$. For both binary and ternary mixtures about 30 samples were used in the experiment.

For a binary system, the mass flux contribution by molecular diffusion and thermal diffusion was usually written as below:

$$
\mathrm{J}_{1}=-\rho\left(\mathrm{D}^{\mathrm{M}} \nabla \mathrm{C}+\mathrm{D}^{\mathrm{T}} \mathrm{C}_{\mathrm{o}}\left(1-\mathrm{C}_{\mathrm{o}}\right) \nabla \mathrm{T}\right)
$$

where, $\rho$ is the mixture mass density; $\mathrm{C}$ is the mass fraction of component 1 with initial value $\mathrm{C}_{0} ; \mathrm{J}_{1}$ is the mass flux of component 1 in the binary mixture.

The molecular diffusion coefficient $\mathrm{D}^{\mathrm{M}}$ was measured based on the open-ended capillary or OEC technique, also described in detail by Dutrieux et al. [41].

For ternary systems there are two independent mass fluxes, $\mathrm{J}_{1}$ and $\mathrm{J}_{2}$, and can be written as: 


$$
\left.\begin{array}{l}
J_{1}=-\rho\left(D_{11}^{M} \nabla C_{1}+D_{12}^{M} \nabla C_{2}+D_{T}^{l} \nabla T\right) \\
J_{2}=-\rho\left(D_{21}^{M} \nabla C_{1}+D_{22}^{M} \nabla C_{2}+D_{T}^{2} \nabla T\right)
\end{array}\right\}
$$

where, $C_{2}$ is the mass fraction of component $2 ; D_{T}^{1}$ and $D_{T}^{2}$ are the thermal diffusion coefficients of component 1 and 2 respectively. Since the units used in Firoozabadi's model and Platten's experimental data are different, the conversion was made for a correct comparison. (Please see Appendix A for details.)

\subsection{RESULTS AND DISCUSSION}

In order to accurately compare the thermal and molecular diffusion coefficients for three binary mixtures, an attempt was made to calculate the density of mixture $\mathrm{THN}-\mathrm{C}_{12} \mathrm{H}_{26}$, IBB- $\mathrm{C}_{12} \mathrm{H}_{26}$, and THN-IBB and compare the results with the experimental data available in the literature. The critical properties of the pure components utilized in the calculation of density using PR-EOS are shown in Table 1.

Table 1: The critical properties of pure components

\begin{tabular}{lllll}
\hline & Asentric factor $\omega$ & Critical T $(\mathrm{K})$ & Critical P $(\mathrm{Pa})$ & Critical Volume $\left(\mathrm{m}^{3} / \mathrm{kg}\right)$ \\
\hline THN & 0.3030 & 719.00 & $0.3515 \times 10^{7}$ & $3.335 \times 10^{-3}$ \\
Dodecane & 0.5764 & 658.20 & $0.1820 \times 10^{7}$ & $4.203 \times 10^{-3}$ \\
IBB & 0.3780 & 650.00 & $0.3141 \times 10^{7}$ & $3.577 \times 10^{-3}$ \\
\hline
\end{tabular}

Using the PR equation of state, the density of the mixtures was calculated and shown in Table 2 at a pressure of 1 bar and a temperature of $298 \mathrm{~K}$.

Table 2: The density calculation at $P=1$ bar and $\mathrm{T}=298 \mathrm{~K}$

\begin{tabular}{lccc}
\hline & THN- $\mathrm{C}_{12} \mathrm{H}_{26}\left(\mathrm{~g} / \mathrm{cm}^{3}\right)$ & $\mathrm{IBB}-\mathrm{C}_{12} \mathrm{H}_{26}\left(\mathrm{~g} / \mathrm{cm}^{3}\right)$ & $\mathrm{THN}-\mathrm{IBB}\left(\mathrm{g} / \mathrm{cm}^{3}\right)$ \\
\hline Experimental & 0.8416 & 0.7925 & 0.9046 \\
Theoretical & 0.8029 & 0.8000 & 0.9032 \\
\hline
\end{tabular}




\begin{tabular}{llll}
\hline Percentage error & $4.59 \%$ & $0.95 \%$ & $0.15 \%$
\end{tabular}

As shown for all three binary mixtures, a strong agreement exists between the experimental data and values obtained by the theoretical technique. The slight deviation in values can be attributed to the fact that the Peng-Robinson equation of state approach is an approximation technique; hence, some inaccuracy can be expected. Furthermore, the density prediction may be improved by introducing a value for the volume-translation parameters for the IBB and THN. In this study, because they were difficult to obtain, the volume-translation was assumed to be zero. To further enhance the confidence in the density prediction, the temperature and mass concentration were plotted. Figure 1, Figure 2, and Figure 3 present the density variation of three binary mixtures with temperature. Figure 4, Figure 5, and Figure 6 present the density of the three binary mixtures as a function of mass concentration.

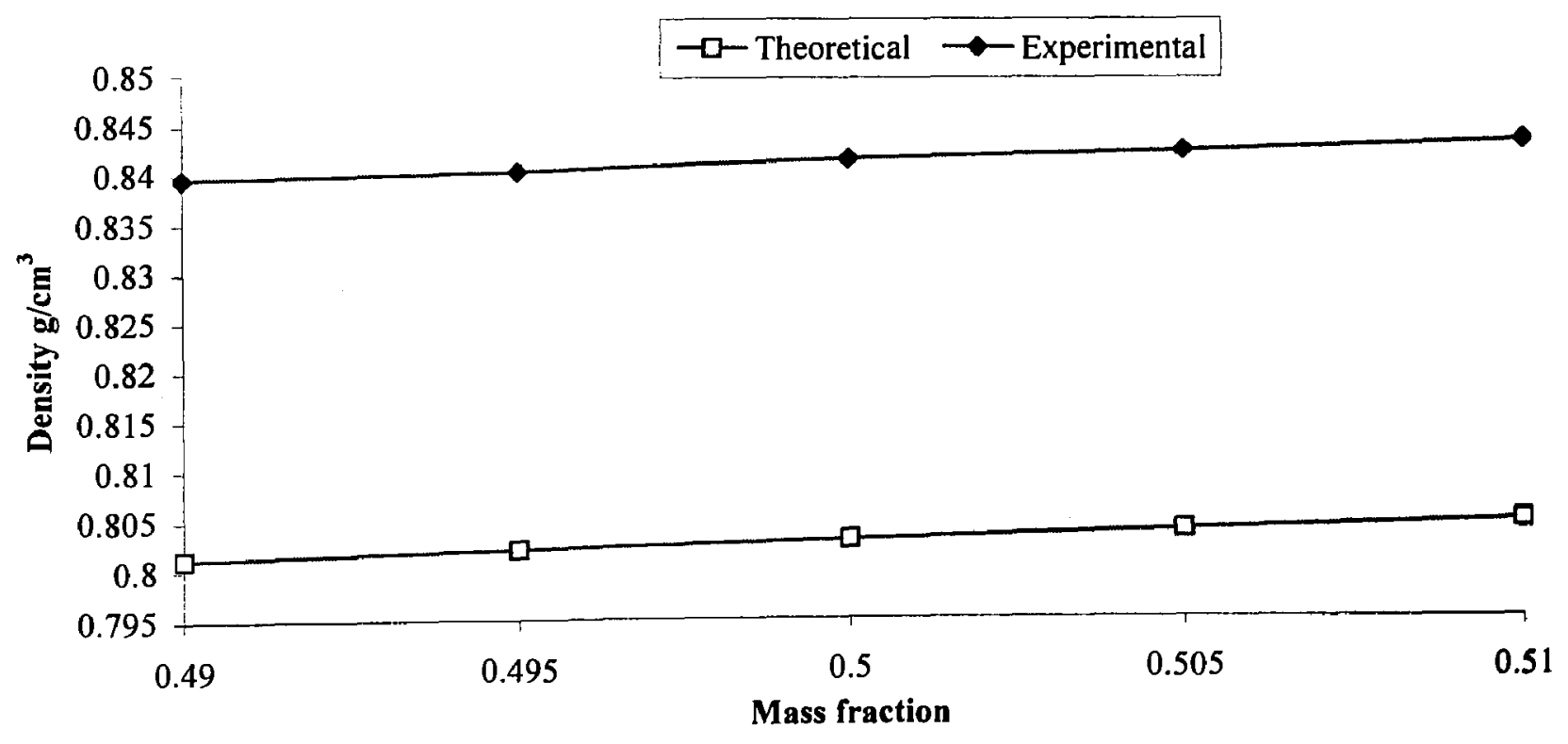

Figure 1: Density variation as function of THN percentage for THN-C $\mathrm{C}_{12} \mathrm{H}_{26}$ mixture 


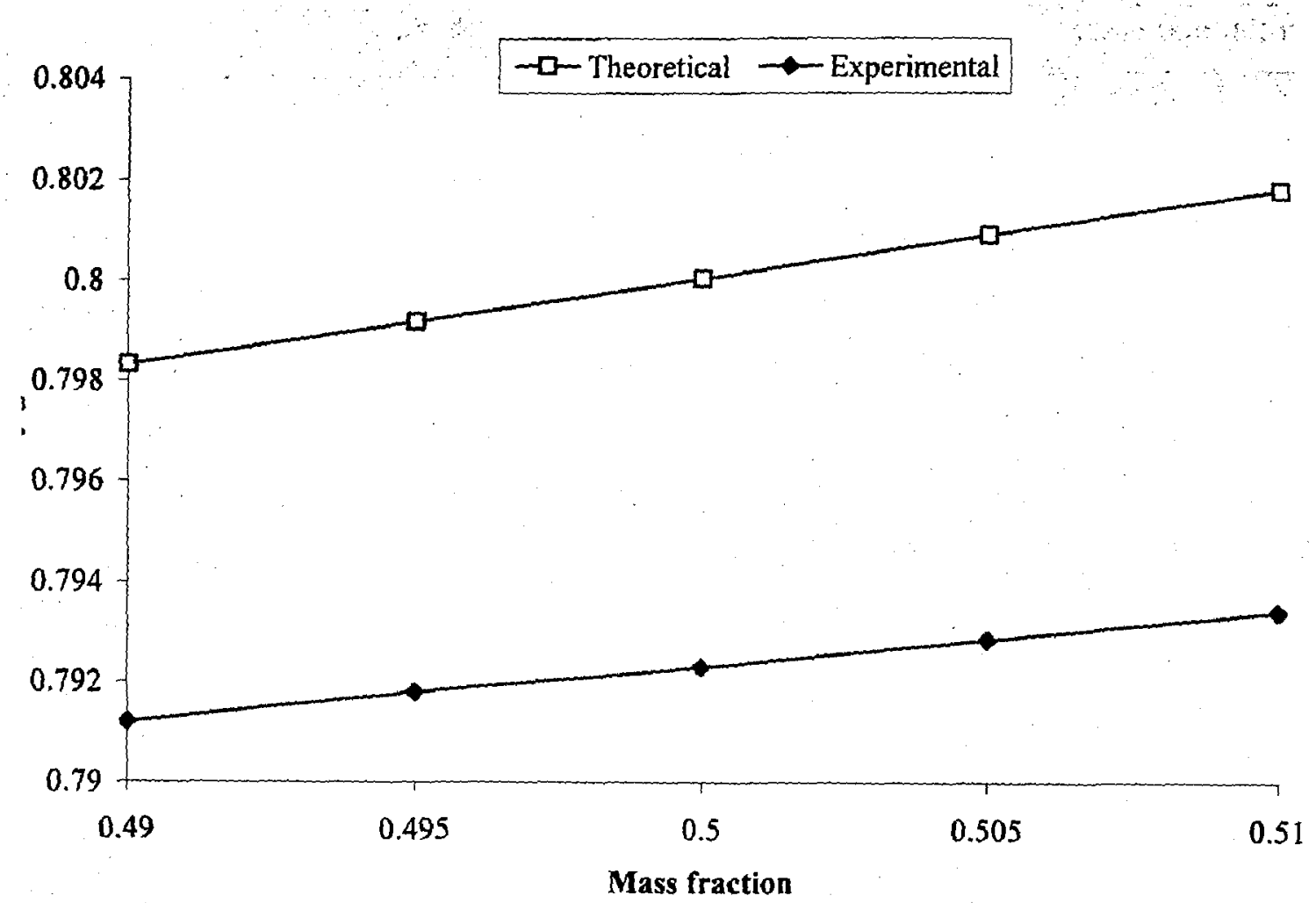

Figure 2: Density variation as function of $\mathrm{IBB}$ percentage for $\mathrm{IBB}-\mathrm{C}_{12} \mathrm{H}_{26}$ mixture 


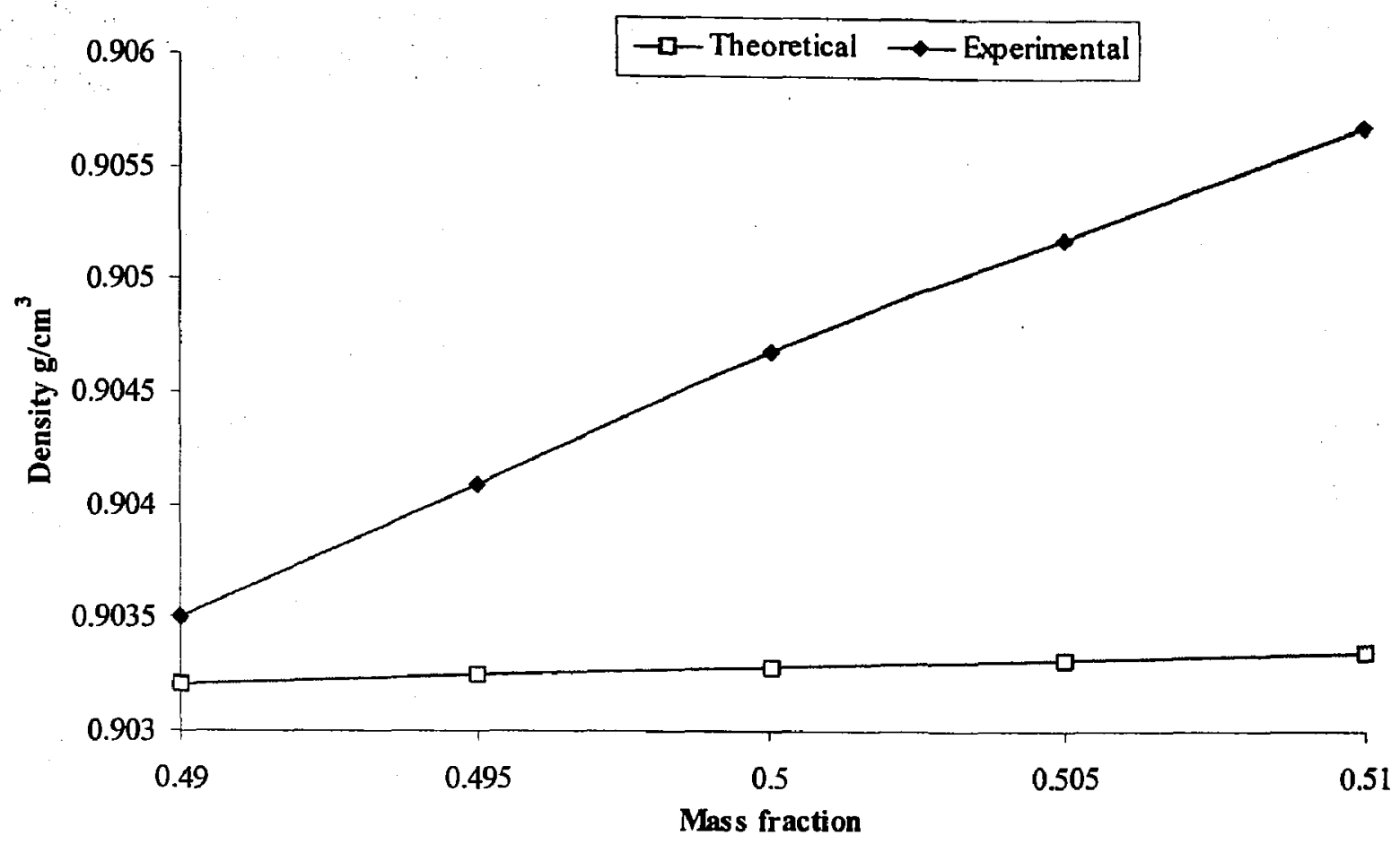

Figure 3: Density variation as function of THN percentage for THN-IBB mixture

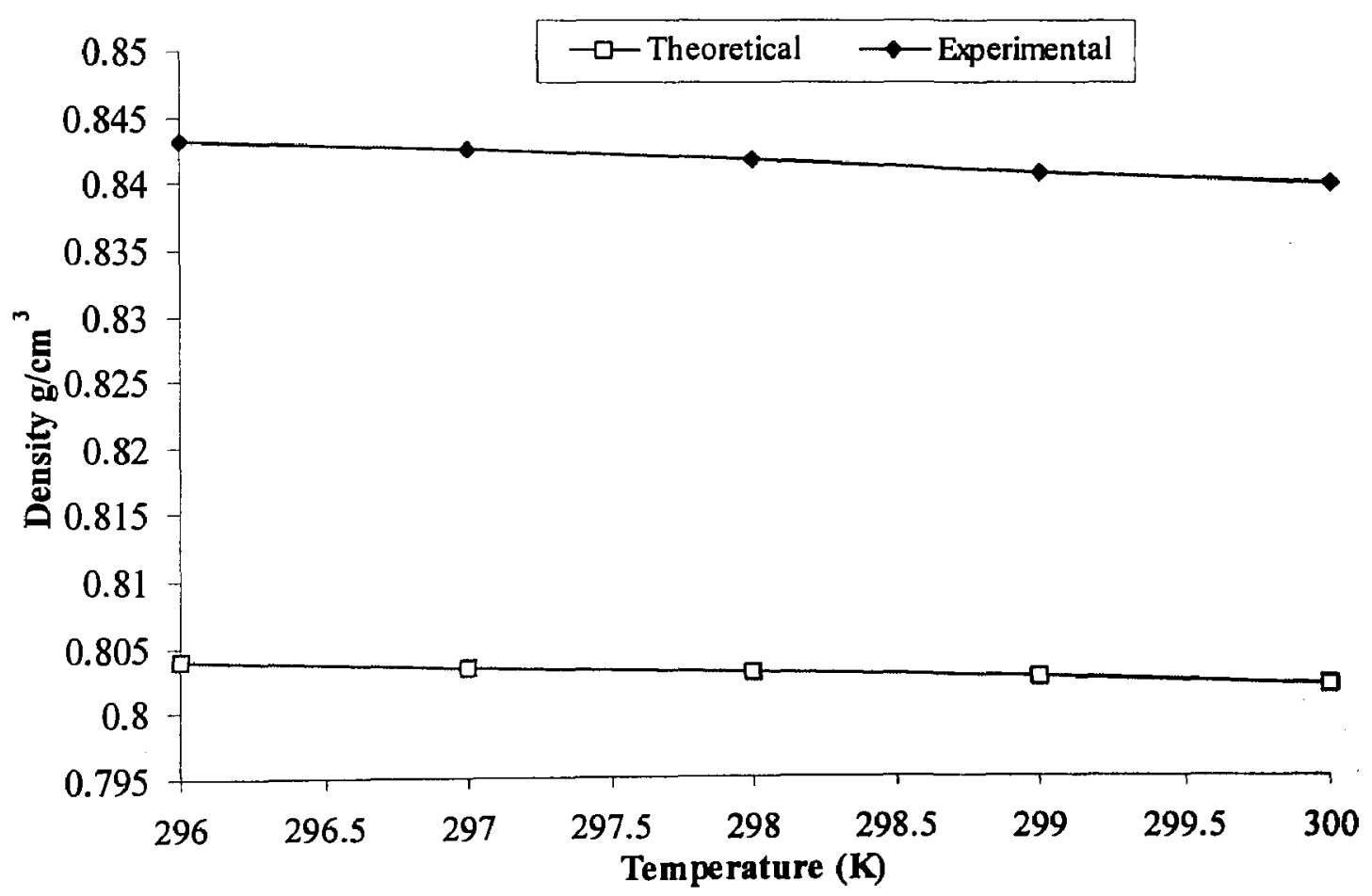

Figure 4: Density variation of THN- $\mathrm{C}_{12} \mathrm{H}_{26}$ mixture 


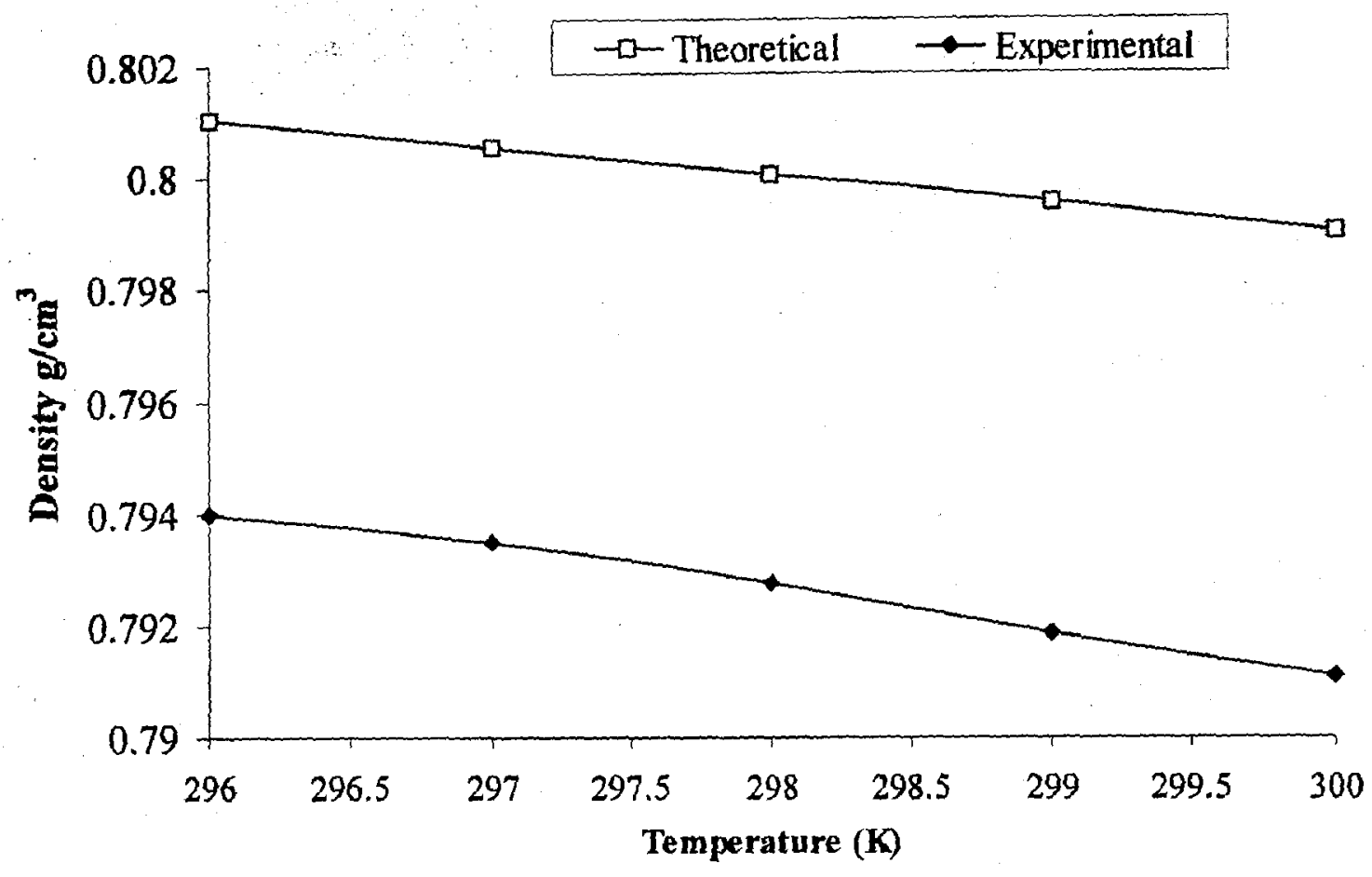

Figure 5: Density variation of $\mathrm{IBB}-\mathrm{C}_{12} \mathrm{H}_{26}$ mixture

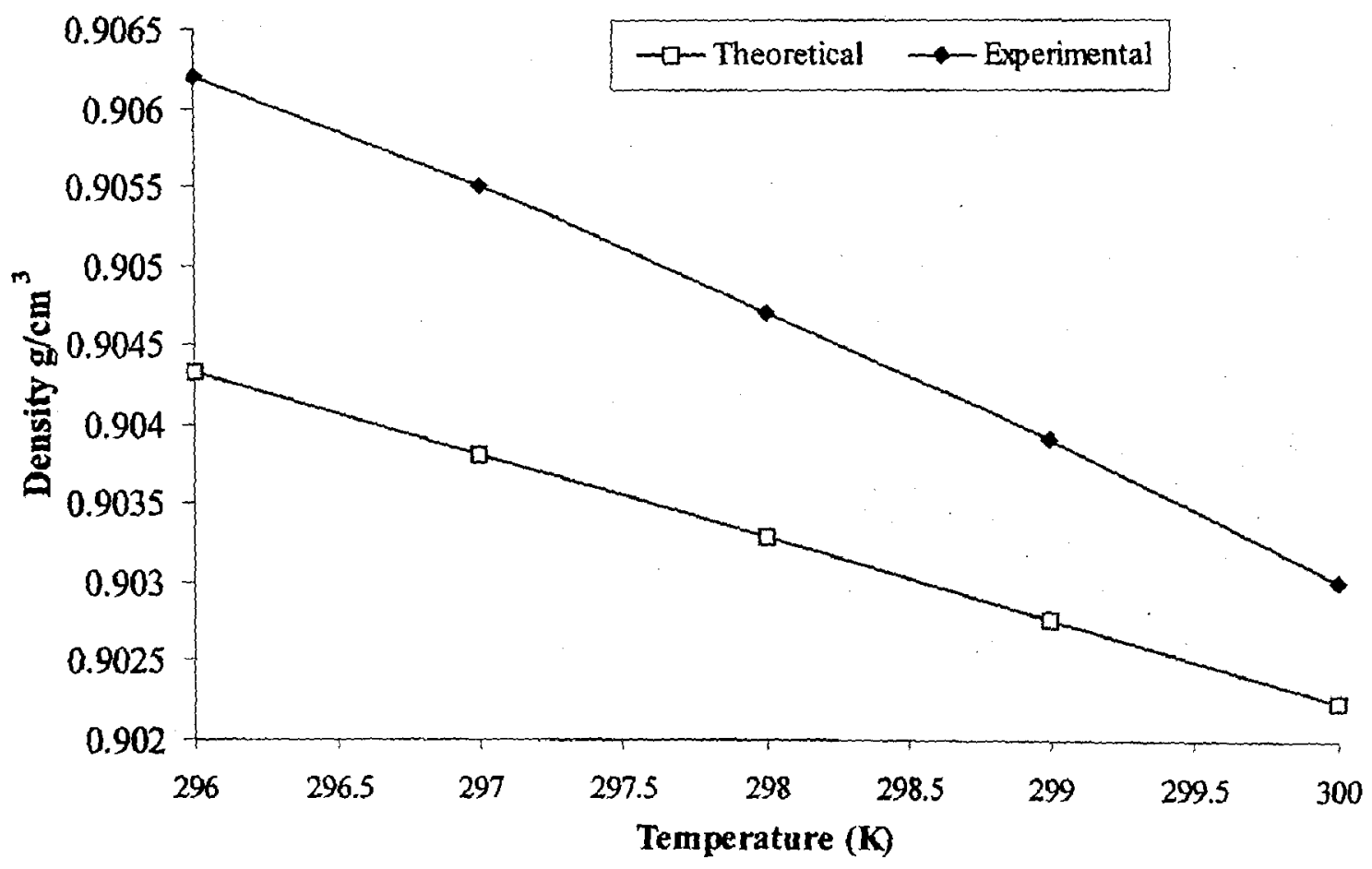

Figure 6: Density variation of THN-IBB mixture 
It can be noted that all curves show a similar variation but differ in values; however, the difference is less than $\pm 5 \%$. Therefore, there exists a strong assurance in the PR equation of state's ability to predict the density for hydrocarbon mixtures. This will be used in calculations of molar density, molar volume, fugacity and enthalpy. In turn, this will be used to calculate the thermal and molecular diffusion coefficients.

Table 3 presents the data for molecular diffusion coefficients, and Table 4 presents the data for thermal diffusion coefficients for both experiment and theoretical techniques.

Table 3: Theoretical and Experimental Molecular diffusion coefficients for three binary mixtures

\begin{tabular}{lccc}
\hline & THN- $\mathrm{C}_{12} \mathrm{H}_{26}\left(\mathrm{~m}^{2} / \mathrm{s}\right)$ & $\mathrm{IBB}-\mathrm{C}_{12} \mathrm{H}_{26}\left(\mathrm{~m}^{2} / \mathrm{s}\right)$ & THN-IBB $\left(\mathrm{m}^{2} / \mathrm{s}\right)$ \\
\hline Experimental & $6.210 \times 10^{-10}$ & $9.500 \times 10^{-10}$ & $8.500 \times 10^{-10}$ \\
Theoretical & $6.044 \times 10^{-10}$ & $7.229 \times 10^{-10}$ & $9.536 \times 10^{-10}$ \\
Percentage error & $2.68 \%$ & $23.91 \%$ & $12.19 \%$ \\
\hline
\end{tabular}

Table 4: Theoretical and Experimental Thermal diffusion coefficients for three binary mixtures

\begin{tabular}{|c|c|c|c|}
\hline & $\mathrm{THN}-\mathrm{C}_{12} \mathrm{H}_{26}\left(\mathrm{~m}^{2} / \mathrm{s} . \mathrm{K}\right)$ & IBB-C $_{12} \mathrm{H}_{26}\left(\mathrm{~m}^{2} / \mathrm{s} . \mathrm{K}\right)$ & THN-IBB $\left(\mathrm{m}^{2} / \mathrm{s} . \mathrm{K}\right)$ \\
\hline Experimental & $5.900 \times 10^{-12}$ & $3.700 \times 10^{-12}$ & $2.800 \times 10^{-12}$ \\
\hline Theoretical & $5.484 \times 10^{-12}$ & $3.882 \times 10^{-12}$ & $1.139 \times 10^{-12}$ \\
\hline Percentage error & $7.05 \%$ & $4.92 \%$ & $59.33 \%$ \\
\hline
\end{tabular}

The experimental and theoretical thermal and molecular diffusion coefficients for THN$\mathrm{C}_{12} \mathrm{H}_{26}$ are in strong agreement with each other. Furthermore, it is evident that, in the mixture of IBB- $\mathrm{C}_{12} \mathrm{H}_{26}$, the thermal diffusion coefficients are in close agreement with each other. Moreover, even though the theoretical thermal diffusion coefficient for THNIBB is lower than the experimental value, the molecular diffusion coefficients for THNIBB are in close agreement. The difference in values can be attributed to the fact that THN and IBB are atypical fluids. As well, some of the thermodynamics properties such as the molar volume at the boiling point and the viscosity of the pure components, were difficult to attain. Hence, approximate values were utilized. The comparison between the 
experimental and theoretical values can be improved with accurate thermodynamic properties.

Table 5 presents both theoretical and experimental data for thermal diffusion coefficients for the ternary system of $\mathrm{C}_{12} \mathrm{H}_{26}$-THN-IBB. This was done at a fixed concentration of 33 wt $\%$ for each components and maintained at $25{ }^{\circ} \mathrm{C}$ and 1 bar. It is evident that the theoretical thermal diffusion coefficients for THN and $\mathrm{C}_{12} \mathrm{H}_{26}$ are in fairly close agreement with the experimental values. However, the theoretical thermal diffusion coefficient for IBB is larger than the experimental value. Once again, these differences in values can be attributed to the fact that the theoretical values are calculated based on approximate techniques. This is in addition to that fact that some of the thermodynamic properties of the pure components were approximated. The combination of these approximations enlarges the error of the thermal diffusion coefficient for the carrier, which in this system is IBB.

Table 5: Theoretical and Experimental thermal diffusion coefficients for the ternary system

\begin{tabular}{lccc}
\hline & $\mathrm{D}_{\mathrm{THN}}^{\mathrm{T}}\left(\mathrm{m}^{2} / \mathrm{s} . \mathrm{K}\right)$ & $\mathrm{D}_{\mathrm{C}_{12}{ }_{1} \mathrm{H}}{ }_{26}\left(\mathrm{~m}^{2} / \mathrm{s} . \mathrm{K}\right)$ & $\mathrm{D}_{\mathrm{IBB}}^{\mathrm{T}}\left(\mathrm{m}^{2} / \mathrm{s} . \mathrm{K}\right)$ \\
\hline Experimental & $8.740 \times 10^{-13}$ & $-1.021 \times 10^{-12}$ & $1.470 \times 10^{-13}$ \\
Theoretical & $7.291 \times 10^{-13}$ & $-1.221 \times 10^{-12}$ & $4.918 \times 10^{-13}$ \\
Percentage error & $16.58 \%$ & $19.59 \%$ & $234.62 \%$ \\
\hline
\end{tabular}

Platten et al. (2004) [9] suggested considering the thermal diffusion coefficient of a particular component in a ternary system as a linear combination of two thermal diffusion coefficients. This can form from the two binaries, and the two other components and is represented mathematically as follows:

$$
\breve{D}_{i}^{T}(\text { ternary } i+j+k)=D_{i}^{T}(\text { binary } i+j) C_{i}^{o} C_{j}^{o}+D_{i}^{T}(\text { binary } i+k) C_{i}^{o} C_{k}^{o}
$$


For the ternary system of $\mathrm{C}_{12} \mathrm{H}_{26}-\mathrm{THN}-\mathrm{IBB} \mathrm{C}_{\mathrm{i}}^{\mathrm{o}}=\mathrm{C}_{\mathrm{j}}^{\mathrm{o}}=\mathrm{C}_{\mathrm{k}}^{\mathrm{o}}=\frac{1}{3}$. Using the values for the thermal diffusion coefficients (presented in Table 4) in the calculations, the calculated values are presented in Table 6 .

Table 6: Thermal diffusion coefficients calculated using the linear combination

\begin{tabular}{llll}
\hline & $\breve{\mathrm{D}}_{\mathrm{THN}}^{\mathrm{T}}\left(\mathrm{m}^{2} / \mathrm{s} . \mathrm{K}\right)$ & $\breve{\mathrm{D}}_{\mathrm{C}_{12} \mathrm{H}_{26}}^{\mathrm{T}}\left(\mathrm{m}^{2} / \mathrm{s} . \mathrm{K}\right)$ & $\breve{\mathrm{D}}_{\mathrm{IBB}}^{\mathrm{T}}\left(\mathrm{m}^{2} / \mathrm{s} . \mathrm{K}\right)$ \\
\hline Experimental calculated & $9.666 \times 10^{-13}$ & $-1.066 \times 10^{-12}$ & $1.000 \times 10^{-13}$ \\
Theoretical calculated & $7.359 \times 10^{-13}$ & $-1.040 \times 10^{-12}$ & $3.048 \times 10^{-13}$ \\
\hline
\end{tabular}

This calculation yields values that are close to those obtained using both experimental and theoretical techniques. This calculation method can be used as a quick estimation method for obtaining the thermal diffusion coefficients for ternary systems from binary systems.

\subsection{SUMMARY}

By using Fontainebleau's Benchmark values, as well as both Firoozabadi's model and Peng-Robinson's equation of state, the theoretical calculation of thermal and molecular diffusion for three binary mixtures of n-Dodecane $\left(\mathrm{C}_{12} \mathrm{H}_{26}\right)$, Isobutylbenezene (IBB), and 1, 2, 3, 4-Tetrahydronaphthalene (THN) were made. This was done at a fixed concentration of $50 \mathrm{wt} \%$ and at a mean temperature of $25^{\circ} \mathrm{C}$. By using Firoozabadi's model and comparing this with the experimental data, for the first time, the thermal diffusion coefficients for the ternary mixture of $\mathrm{C}_{12} \mathrm{H}_{26}-\mathrm{THN}-\mathrm{IBB}$ was calculated. Also, in the study, the thermal diffusion coefficients in the ternary mixture were successfully correlated with the thermal diffusion coefficients from the three binary mixtures as suggested by Platten et al. [9]. The results revealed a strong agreement between the experimental and numerical data. The results can be improved by utilizing accurate thermodynamic properties, such as the molar volume at boiling point, and the viscosity of the pure component. 


\section{CHAPTER 4: TWO DIMENSIONAL SIMULATION OF THERMAL DIFFUSION IN POROUS MEDIA}

\subsection{CONVECTION IN POROUS MEDIA}

Convection can be explained as the transfer of heat by the motion of or within fluid. Convection may arise from temperature differences either within the fluid or between the fluid and its boundary. The basic premise behind convection is that heated matter becomes more buoyant and rises, while the cooler material sinks. Therefore, when a liquid or gas that expands or contracts in response to changing temperatures when it is exposed to multiple temperatures in an acceleration field such as gravity, convection known as buoyancy-driven convection takes place.

Buoyancy-driven convection can also take place in multicomponent mixtures, and the occurrence is more intricate than in a single component fluid. In multicomponent mixtures, one component may react quicker than the rest, which can cause an oscillatory convection to take place.

Convection also has a great influence on the thermal diffusion process in hydrocarbons contained in a porous media. This chapter investigates numerically the interaction between thermal diffusion and buoyancy driven convection in a laterally heated vertical porous cavity filled with three binary mixtures of THN-Dodecane, IBB-Dodecane, and THN-IBB. An aspect ratio of 10 is selected, because most petroleum applications deal with a relatively large aspect ratio. The thermal diffusion process is simulated in a vertical porous medium with permeability values ranging from $0.001 \mathrm{md}$ to $10,000 \mathrm{md}$. The next section introduces the model and the numerical solution technique employed to solve it.

\subsection{THE NUMERICAL MODEL}


A vertical cavity with height $(\mathrm{H})$ of $5 \mathrm{~m}$ and width $(\mathrm{W})$ of $0.5 \mathrm{~m}$ is constructed with various boundary conditions, as shown in Figure 7. The four walls are assumed to be non-reacting, solid, impermeable, and with the no-slip condition. The two lateral walls have a Dirichlet boundary condition; the right wall with a hot temperature of $303 \mathrm{~K}$, and the left wall with a cold temperature of $293 \mathrm{~K}$. The top and bottom walls are assumed to be adiabatic. The binary mixtures filling the vertical cavity consist of THN-Dodecane, IBB-Dodecane, and THN-Dodecane, each component at 50\% mass fraction and under atmospheric pressure. The fluid is considered to be compressible, with no heat generation, no chemical reaction, and no interactive superficial forces acting between the porous medium particles and liquid mixture.

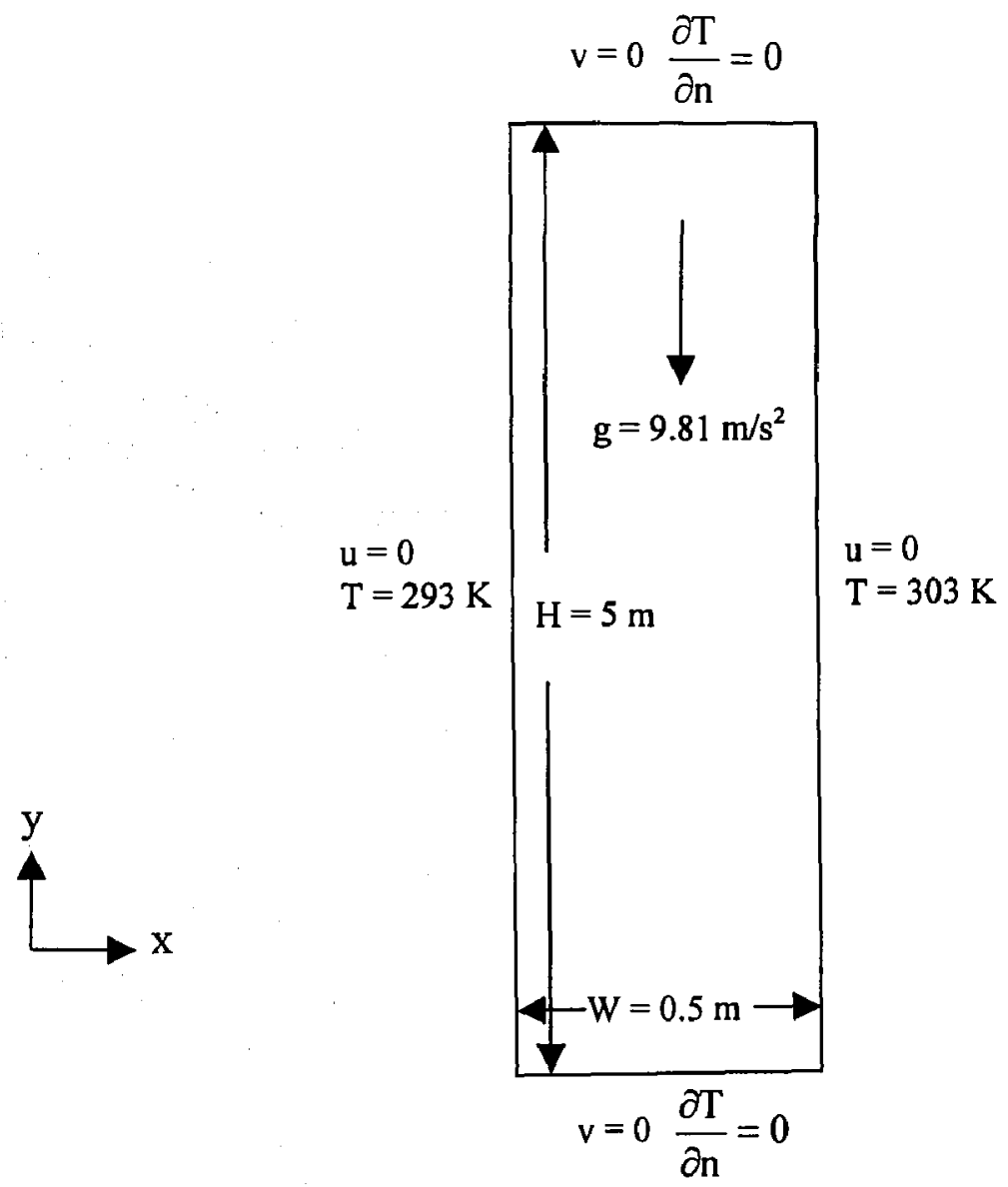

Figure 7: Two-dimensional porous media model and boundary conditions 


\subsection{MATHEMATICAL MODELLING}

In order to simulate the thermal diffusion process in a porous medium cavity, field equations to describe the movement of the mixture fluid to thermo-solutal and gravitational driving forces are required. Therefore, the mass continuity equation, the momentum conservation equation and the energy conservation equation are introduced here.

\subsubsection{Mass Continuity Equation}

In the two-dimensional domain, the general conversation of mass equation is written in the following form:

$$
\frac{\partial \rho_{\mathrm{m}}}{\partial \mathrm{t}}+\frac{\partial\left(\rho_{\mathrm{m}} \mathrm{u}\right)}{\partial \mathrm{x}}+\frac{\partial\left(\rho_{\mathrm{m}} \mathrm{v}\right)}{\partial \mathrm{y}}=0
$$

where, $\rho_{\mathrm{m}}$ is the molar density of the mixture fluid, and $u$ and $v$ are the horizontal and vertical velocity components, respectively. Since a multicomponent mixture is used, an additional mass conversation equation is solved, namely:

$$
\frac{\partial\left(\rho_{\mathrm{m}} \mathrm{c}\right)}{\partial \mathrm{t}}+\frac{\partial\left(\rho_{\mathrm{m}} \mathrm{uc}\right)}{\partial \mathrm{x}}+\frac{\partial\left(\rho_{\mathrm{m}} \mathrm{vc}\right)}{\partial \mathrm{y}}=-\nabla \cdot \overrightarrow{\mathrm{J}}
$$

where, $\overrightarrow{\mathrm{J}}$ is the mass flux of the solute and is shown in general form in Eq. (55), and $\mathrm{c}$ is the concentration of the solute.

\subsubsection{Momentum Conservation Equation}

In porous media, the porous matrix is assumed to be homogenous and isotropic, Therefore, the Darcy's equation presented in Eq. (16), can be applied and expressed in the following form: 


$$
\overrightarrow{\mathrm{v}}=-\frac{\kappa}{\varphi \mu}(\nabla \overrightarrow{\mathrm{P}}+\mathrm{pg})
$$

By substituting the Darcy's equation into the general conservation of mass equation (81), the pressure differential equation becomes as follows:

$$
\frac{\partial \rho_{\mathrm{m}}}{\partial \mathrm{t}}-\frac{\kappa}{\varphi \mu} \frac{\partial}{\partial \mathrm{x}}\left(\rho_{\mathrm{m}} \frac{\partial \mathrm{p}}{\partial \mathrm{x}}\right)-\frac{\kappa}{\varphi \mu} \frac{\partial}{\partial \mathrm{y}}\left(\rho_{\mathrm{n}} \frac{\partial \mathrm{p}}{\partial \mathrm{y}}+\rho \mathrm{g}\right)=0
$$

\subsubsection{Energy Conservation Equation}

The energy conservation equation presented in Eq. (34) can be expressed as follows for a two-dimensional domain and with Darcy's equation substituted in it:

$$
\frac{\partial\left(\rho c_{m}\right) T}{\partial t}+\varphi u \frac{\partial}{\partial x}\left(\left(\rho c_{m}\right)_{f} T\right)+\varphi v \frac{\partial}{\partial y}\left(\left(\rho c_{m}\right)_{f} T\right)=K_{m}\left[\frac{\partial^{2} T}{\partial x^{2}}+\frac{\partial^{2} T}{\partial y^{2}}\right]
$$

\subsection{NUMERICAL SOLUTION TECHNIQUE}

Computational Fluid Dynamics, CFD, is a common analytical tool to tackle various fluid flow problems including the thermal diffusion phenomenon. Before solving a particular flow problem, the geometry of the region of interest must be clearly defined. Additionally, since the accuracy of a CFD solution is sensitive to the mesh size, the mesh must be generated properly and tested. Generally, the finer the mesh is, the more accurate the solution. However, a fine mesh will incur a high cost in terms of the computer hardware and calculation time. Therefore, in practice, there is a need to do mesh sensitivity analysis for an optimal mesh size.

A series of conservation equations (mass, momentum, energy) and equation of state, together-with the appropriate boundary and initial conditions-must be solved numerically. There are several numerical solution techniques, such as finite element and 
finite-difference. Among these, the finite volume method, or, control volume method, is one of the well established and thoroughly validated general purpose CFD techniques. Its numerical algorithm consists of three components. The first is integrating the governing equations of fluid flow over all the control volumes of the solution domain. The second component involves converting the governing equations into discrete equations at its nodal points by applying finite-difference-type approximations for the terms. This represents flow processes such as convection, diffusion and sources in the integrated equations. Lastly, the third component involves solving the discrete equations using appropriate iterative methods. The control volume is constrained by the law of conservation, i.e., for a general flow variable $\xi$ such as a velocity component or enthalpy, the rate of change of $\xi$ within the finite control volume with respect to time equals to the sum of the net flux of $\xi$ due to both convection and diffusion into the control volume and the net rate of creation of $\xi$ inside the control volume.

The problem of thermodiffusion is associated with non-linearities in the equation set and is strongly coupled since the density is related to the pressure, temperature and composition. Such a problem can be resolved by adopting an iterative solution strategy such as the SIMPLE algorithm of Patankar and Spalding [33]. In this algorithm, using guessed initial pressure and velocity fields starts the iteration process. Then, by solving the continuity equation, a pressure correction field is obtained, which, in turn, is used to update the velocity and pressure field. This process is iterated until the velocity and pressure fields are converged. For two-dimensional cases, a pressure correlation equation may be expressed as follows:

$$
\mathrm{p}=\mathrm{p}^{*}+\mathrm{p}^{\prime}
$$

where $\mathrm{p}^{*}$ is a guessed (or 'incorrect') pressure field and $\mathrm{p}^{\prime}$ is the pressure correction. The same method can be applied to the velocity components: 


$$
u=u^{*}+u^{\prime}, \quad v=v^{*}+v^{\prime}
$$

where $u^{*}$ and $v^{*}$ are the solutions of the momentum equations corresponding to the guessed pressure field $\mathrm{p}^{*}$ and $\mathrm{u}^{\prime}$ and $\mathrm{v}^{\prime}$ are the responses to the pressure corrections to produce the exact velocity field represented by $u$ and $v$. Assuming:

$$
\mathrm{u}^{\prime}=-\mathrm{K}_{\mathrm{x}} \frac{\partial \mathrm{p}^{\prime}}{\partial \mathrm{x}}, \quad \mathrm{v}^{\prime}=-\mathrm{K}_{\mathrm{y}} \frac{\partial \mathrm{p}^{\prime}}{\partial \mathrm{y}}
$$

and substituting the resulting expressions for $\mathrm{u}$ and $\mathrm{v}$ into the continuity equation yields the equation for the pressure-correction $\mathrm{p}^{\prime}$, namely

$$
\frac{\partial}{\partial x}\left[\rho K_{x}\left(\frac{\partial p^{\prime}}{\partial x}\right)\right]+\frac{\partial}{\partial y}\left[\rho K_{y}\left(\frac{\partial p^{\prime}}{\partial y}\right)\right]=\frac{\partial \rho}{\partial t}+\frac{\partial}{\partial x}\left(\rho u^{*}\right)+\frac{\partial}{\partial y}\left(\rho v^{*}\right)
$$

where $\mathrm{K}_{\mathrm{x}}$ and $\mathrm{K}_{\mathrm{y}}$ are simply coefficients that result from the discretization scheme. Further details in the derivation of the pressure and velocity corrections approach may be found in Patankar [34], Peyret and Taylor [35], and Hirsch [36].

Many researchers, such as Jiang et al. [37, 38], Faruque et al. [39], and Chacha and Saghir [16] have used SIMPLE in their studies. For example, Chacha and Saghir [16] applied this algorithm in simulating double-diffusion process for a binary mixture of Methane and n-Butane. They also summarized the main steps of the numerical procedure derived from the SIMPLE algorithm:

Step 0 Set problem configuration, boundary and working/initial conditions. 
Step 1 Determine the average density of the mixture $\rho$ by solving the EOS of choice for the working condition defined by the set $\left(\mathrm{p}_{\mathrm{T}_{\mathrm{m}}}, \mathrm{T}_{\mathrm{m}}, \mathrm{C}_{\mathrm{o}}\right)$. The obtained average value is kept unchanged during the course of the calculation.

Step 2 Increment the time: $t=(k+1) \times \Delta t$.

Step 3 Guess the pressure field $\mathrm{p}^{*}=\mathrm{p}_{\mathrm{T}_{\mathrm{m}}}$.

Step 4 Determine the mass diffusion coefficient $D^{M}$, the thermal diffusion coefficient $\mathrm{D}^{\mathrm{T}}$ and the viscosity $\mu$. Formulation for these coefficients may be referred to Chacha et al. [19] and the viscosity to Faruque et al. [39]. The values of the transport coefficients at the interface between two control volumes are harmonic means of the values calculated at the adjacent (main) grid points.

Step 5 Solve the momentum equations to obtain the velocity components $\mathrm{u}$ and $\mathrm{v}$ by applying the boundary conditions; these are the starting values for $\mathrm{u}^{*}$ and $\mathrm{v}^{*}$, respectively. A relaxation factor may be introduced in the algebraic equations before solving. Compute $\phi_{\mathrm{u}}$ and $\phi_{\mathrm{v}}$.

Step 6 Solve the pressure-correction equation (89) for $\mathrm{p}^{\prime}$.

Step 7 Calculate $p$ by adding $\mathrm{p}^{\prime}$ (part or whole) to $\mathrm{p}^{*}: \mathrm{p}=\mathrm{p}^{*}+\alpha_{\mathrm{p}} \mathrm{p}^{\prime} . \alpha_{\mathrm{p}}$ is the pressure relaxation factor.

Step 8 Correct $\mathrm{u}$ and $\mathrm{v}$ from their starting values using the velocity-correction formulas equation (88).

Step 9 Solve the energy equation to update the temperature profile $\mathrm{T}$ by applying appropriate boundary conditions. Compute $\phi_{\mathrm{T}}$. 
Step 10 Solve the species mass conservation equation to update the mass fraction $\mathbf{C}$ subject to boundary conditions. Compute $\phi_{C}$.

Step 11 Use $\mathrm{p}$ as the new guessed pressure $\mathrm{p}^{*}$, return to step 3 and repeat the whole procedure until a converged solution is obtained. The solution convergence is achieved at each time step once the maximum of the average relative errors in $\mathrm{u}$, $\mathrm{v}, \mathrm{c}$, and $\mathrm{T}$ throughout the mesh is less than a given value, i.e. $\max \left(\phi_{u}, \phi_{v}, \phi_{T}, \phi_{C}\right)<10^{-6}$, say, for two successive iterations. The average relative error is defined by

$$
\phi_{F}=\frac{1}{(n \times m)} \sum_{i=1}^{i=m l} \sum_{j=1}^{j=n}\left|\left(F_{i, j}^{k, s+1}-F_{i, j}^{k, s}\right) / F_{i, j}^{k, s+1}\right|
$$

where $\mathrm{F}$ stands for the unknown $\mathrm{u}, \mathrm{v}, \mathrm{T}$ or $\mathrm{C}, \mathrm{k}$ denotes the time step, and $\mathrm{s}$ is the iteration number. The grid point is specified by its mesh coordinates $(i, j)$.

Step 12 Return to step 2 to calculate for next time step or end the program.

\subsection{MESH SENSITIVITY ANALYSIS}

To simulate a physical flow problem, it is crucial to create a model of the flow domain. The accuracy of the solution for the flow problem is dependent on the finite element mesh. Fundamentally, the finite element mesh is an idealization of the model geometry. It involves defining a discrete point called the node, and a collections of points called the elements, which the numerical procedure then uses to solve the appropriate flow equations. The accuracy of the results is often dependent on the quality of the finite element mesh. While having a coarse mesh may lead to erroneous results, having a very fine mesh can be very memory-intensive. Thus, it is imperative to choose a suitable mesh size. There are several ways of determining such suitability. These include comparing the steady-state average Nusselt number, for example, or, any other required output, such as the ratio of final concentration to the initial concentration at different mesh sizes. Once 
the error between the two output values is less than an acceptable value, say, $1 \%$ or the value asymptote to a particular value, the optimal mesh size is then selected.

In the case of a porous medium, both the flow and the natural convection are usually very weak. As a result, the Nusselt number does not change very much, thus making it very difficult to determine the optimal mesh size based on the Nusselt number. Alternatively, the ratio of final concentration, $\mathrm{C}$, to the initial concentration, $\mathrm{C}_{0}$, of one of the components may be used to accomplish the mesh sensitivity.

Take the Methane and n-Butane mixture as an example. The mixture is subject to a lateral heating condition. The cold wall is at the temperature of $334 \mathrm{~K}$ and the hot wall at $344 \mathrm{~K}$. The cavity is $5 \mathrm{~m}$ long by $5 \mathrm{~m}$ wide with porosity 0.50 , permeability $10 \mathrm{md}$ and the initial concentration of methane is 0.20 (molar fraction). The final concentration of methane refers to its concentration at the centerline of the cavity along the width of the cavity when the process has reached steady state.

Figure 8 shows the concentration ratios of methane at different mesh sizes. It is apparent that mesh $40 \times 40,50 \times 50$ and $80 \times 80$ give nearly identical curves compared to coarser ones of $5 \times 5,10 \times 10$ and $20 \times 20$. With both accuracy and cost-effectiveness in mind, it was decided that the mesh $40 \times 40$ was in fact the optimal mesh size. The same procedure has been applied to the case studies presented in the section Results and Discussion. 


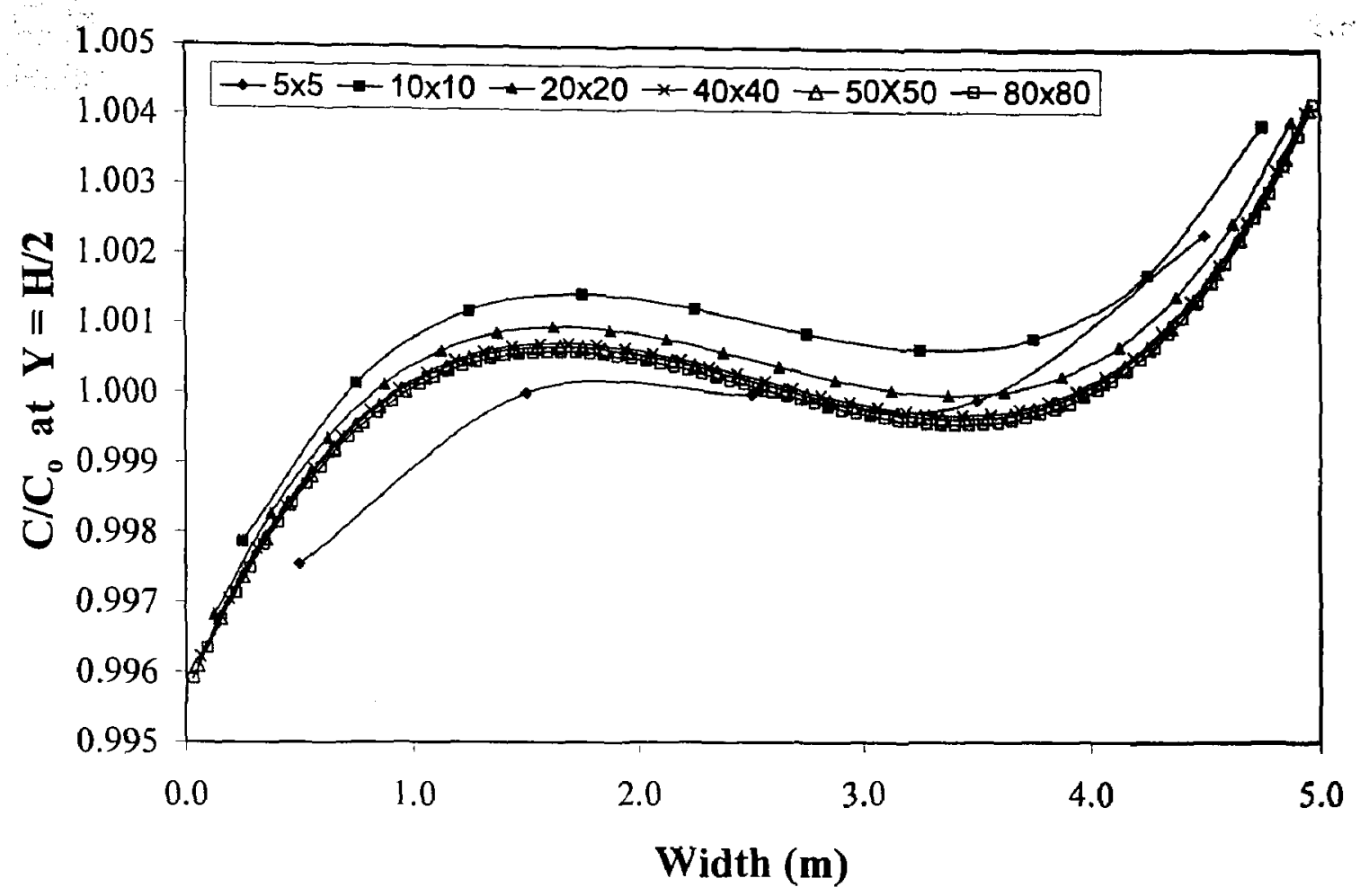

Figure 8: Mesh sensitivity analysis

\subsection{RESULTS AND DISCUSSION}

The present objective is to investigate interaction between thermal diffusion and buoyancy driven convection using a laterally heated vertical porous cavity filled with three binary mixtures of THN-Dodecane, IBB-Dodecane, and THN-IBB. In order to make the analyses more realistic, a wide range of permeabilities is utilized. The permeability ranges from $0.001 \mathrm{md}$ to $10,000 \mathrm{md}$, corresponding to a Darcy number of $3.91 \times 10^{-20}$ to $3.91 \times 10^{-13}$ respectively. The numerical simulation is used to analyze the flow characteristics, the velocity in the cavity, the component migration, and concentration gradients. The separation ratio is also calculated and compared with the analytical value. 


\subsubsection{Comparison of flow patterns}

The streamlines indicating the flow behaviours for THN-Dodecane, IBB-Dodecane, and THN-IBB are depicted in Figure 9, Figure 11, and Figure 13, respectively.

It was noticed in the streamlines that, for all three binary mixtures that are at lower permeabilities, the flow is very weak. The weak flow is further evident in the Figure 10, Figure 12, and Figure 14, which show the velocities along the center of the cavity for the three binary mixtures. The streamline pattern further indicates that the Soret effect is very strong at lower permeability values. On the other hand, it was noticed that, as the permeability increases, the flow gains strength in all three binary mixtures. As shown in Figure 10, Figure 12, and Figure 14, when the permeability increases the fluid flow becomes stronger and causes the fluid to go up along the hot wall and then down along the cold wall, creating a counter-clockwise flow in the cavity. The streamlines also indicate that the buoyancy-driven convection is becoming dominant at higher ranges of permeability.

The flow behaviour in all three mixtures can be explained by realizing that, at lower permeability, the porous medium is tightly packed. The porous medium has a texture similar to unweathered (fat) clay, which makes it difficult for the fluid mixture to flow readily, hence, controlling or reducing the convection. The lateral heat applied to the cavity containing the porous medium creates a temperature gradient inside the cavity, which causes the thermal diffusion to occur. The thermal diffusion or the Soret effect is therefore dominant in the cavity at lower permeabilities. As the permeability increases, the porous media becomes more pervious. It assumes a texture similar to very fine sand. At even higher permeability the porous media becomes almost like well sorted gravel. Therefore, the porous medium becomes looser and allows the mixtures to flow well in it, contributing to the higher fluid velocities in the cavity. The buoyancy-driven convection becomes dominant in the cavity, which in turn has a negative effect on the thermal diffusion. At high permeability values, the convection completely overwhelms the thermal diffusion and the mixture mixes together. 
One of the differences noticed between the three binary mixtures was that the highest velocity among the three mixtures was found in the THN-Dodecane mixture, while the lowest velocity was found in the THN-IBB mixture. In fact, the velocity changed slightly at the higher permeability ranges for the THN-IBB mixture, because the mixture is very viscous when mixed together. 
Figure 9: THN-Dodecane: Streamline contours

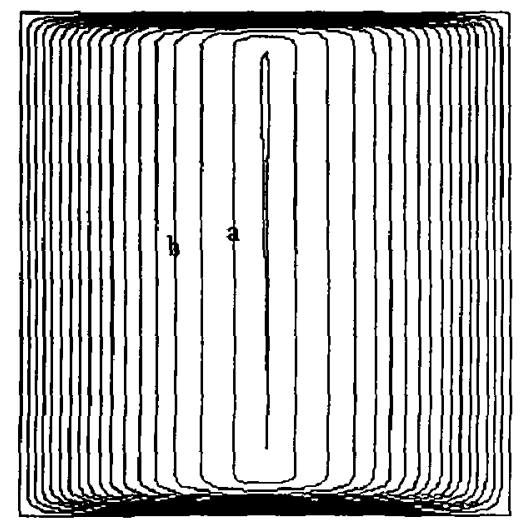

a). $\mathrm{k}=0.001 \mathrm{md}$ $\mathrm{a}=3.007 \times 10^{-11}$ $\mathrm{b}=2.669 \times 10^{-11}$ $\Delta \psi=3.380 \times 10^{-12}$

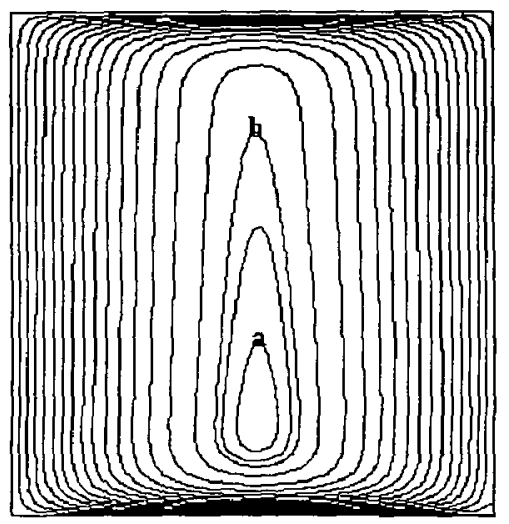

b). $\mathrm{k}=10 \mathrm{md}$ $a=3.029 \times 10^{-7}$ $\mathrm{b}=2.945 \times 10^{-7}$ $\Delta \psi=8.440 \times 10^{-8}$

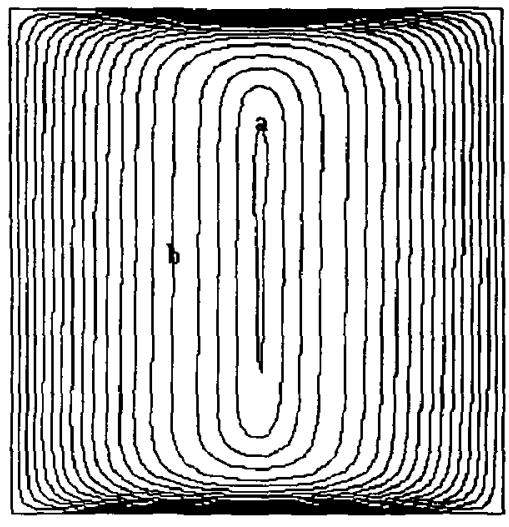

c). $\mathrm{k}=10,000 \mathrm{md}$ $a=3.758 \times 10^{-4}$ $b=1.538 \times 10^{-4}$ $\Delta \psi=2.220 \times 10^{-5}$

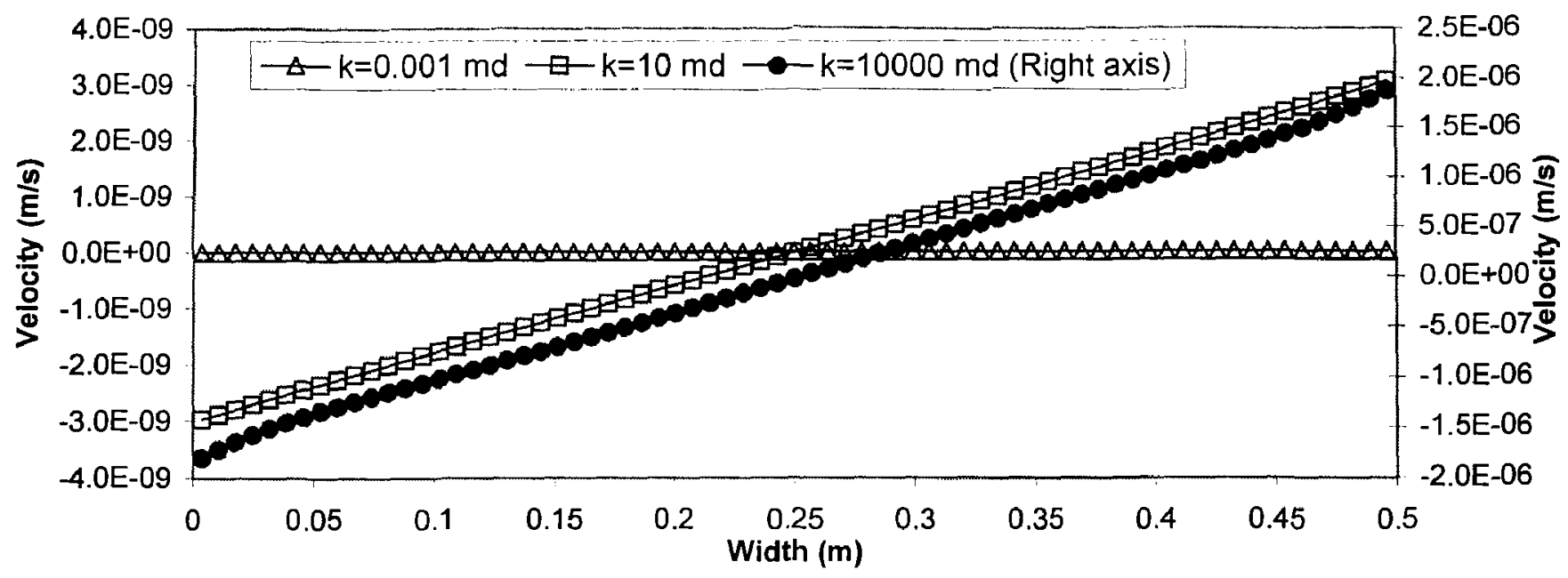

Figure 10: THN-Dodecane: Velocity along the center of the cavity 
Figure 11: IBB-Dodecane: Strea mline contours

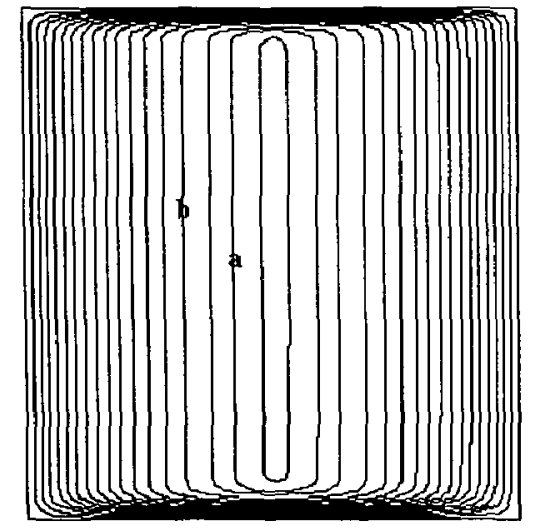

a). $\mathrm{k}=0.001 \mathrm{md}$ $\mathrm{a}=1.954 \times 10^{-11}$

$\mathrm{b}=1.769 \times 10^{-11}$

$\Delta \psi=1.176 \times 10^{-12}$

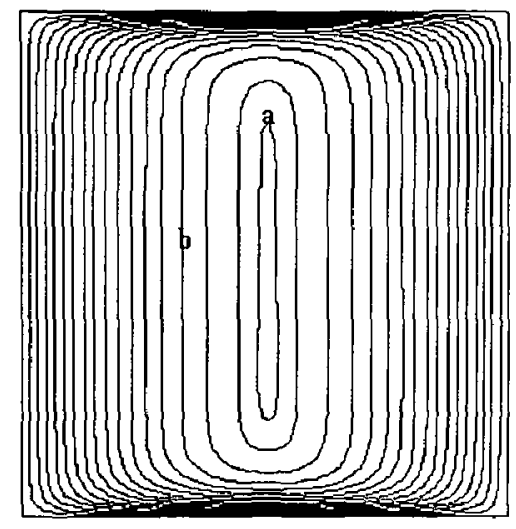

b). $\mathrm{k}=10 \mathrm{md}$ $\mathrm{a}=2.113 \times 10^{-7}$ $\mathrm{b}=1.853 \times 10^{-7}$ $\Delta \psi=1.238 \times 10^{-8}$

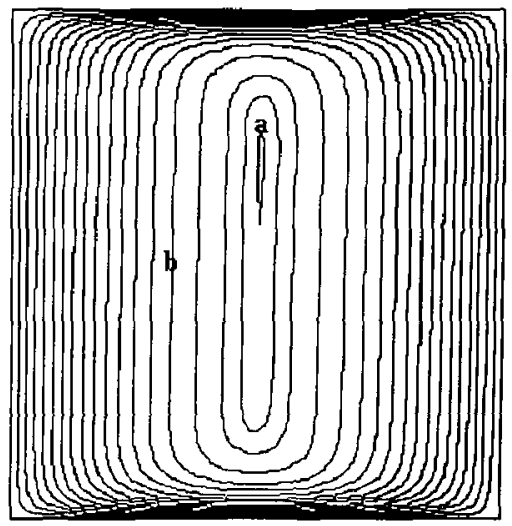

c). $\mathrm{k}=10,000 \mathrm{md}$ $\mathrm{a}=1.484 \times 10^{-4}$ $\mathrm{b}=1.206 \times 10^{-4}$ $\Delta \psi=8.698 \times 10^{-6}$

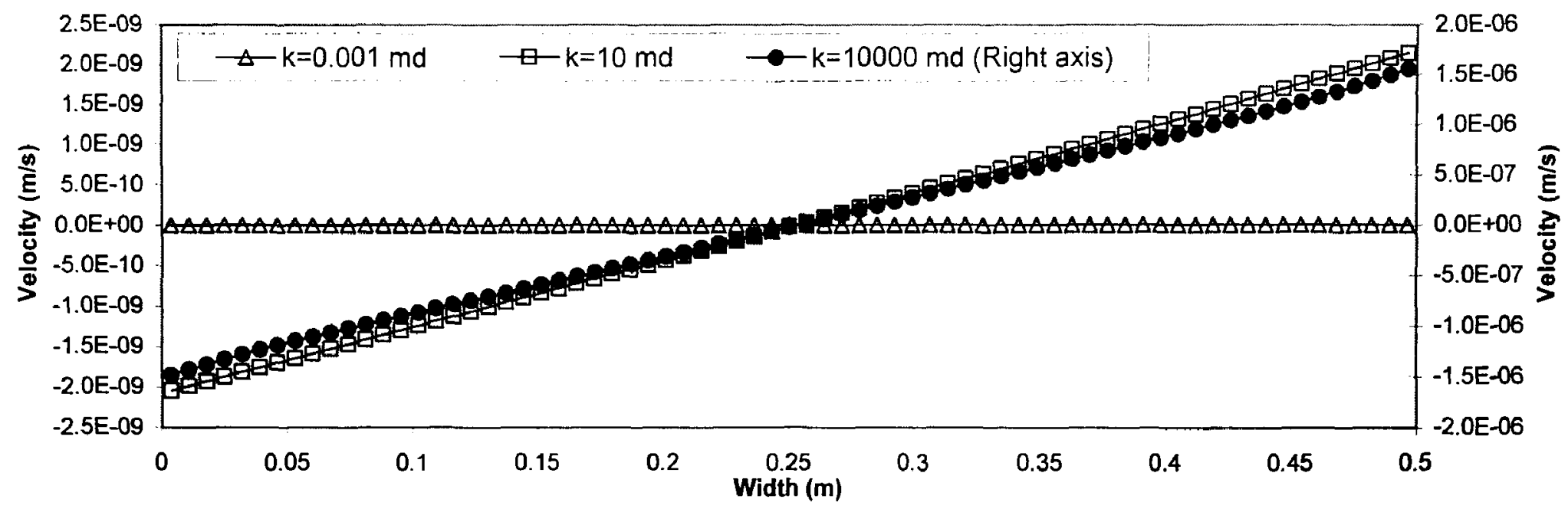

Figure 12: IBB-Dodecane: Velocity along the center of the cavity 
Figure 13: THN-IBB: Streamline contours

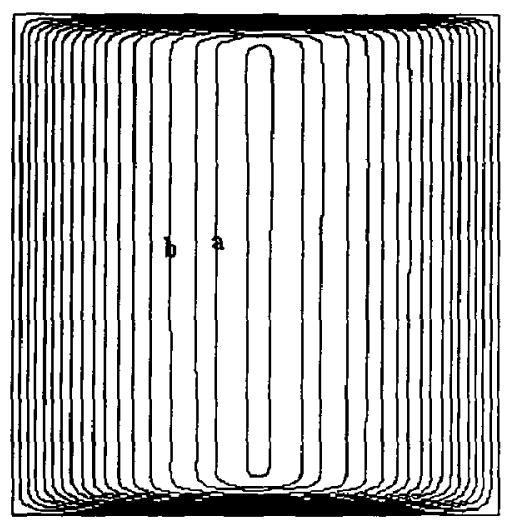

a). $k=0.001 \mathrm{md}$ $\mathrm{a}=1.398 \times 10^{-1 \mathrm{I}}$

$\mathrm{b}=1.315 \times 10^{-11}$

$\Delta \psi=8.194 \times 10^{-13}$

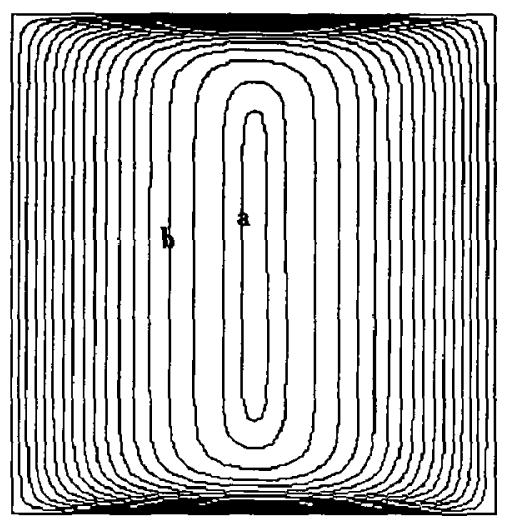

b). $\mathrm{k}=10 \mathrm{md}$ $\mathrm{a}=1.506 \times 10^{-7}$ $\mathrm{b}=1.227 \times 10^{-7}$ $\Delta \psi=8.819 \times 10^{-9}$

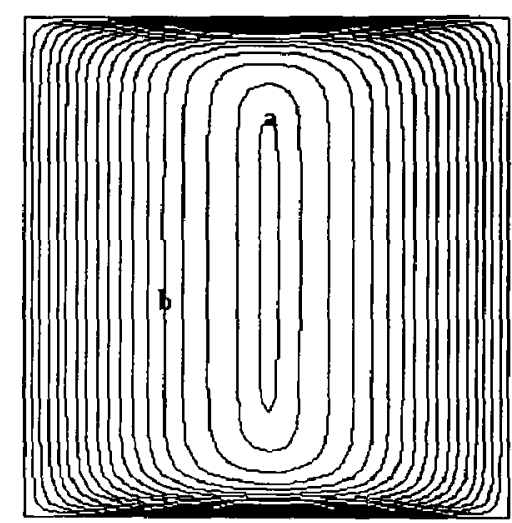

c). $\mathrm{k}=10,000 \mathrm{md}$ $\mathrm{a}=1.503 \times 10^{-4}$ $b=1.222 \times 10^{-4}$ $\Delta \psi=8.802 \times 10^{-6}$

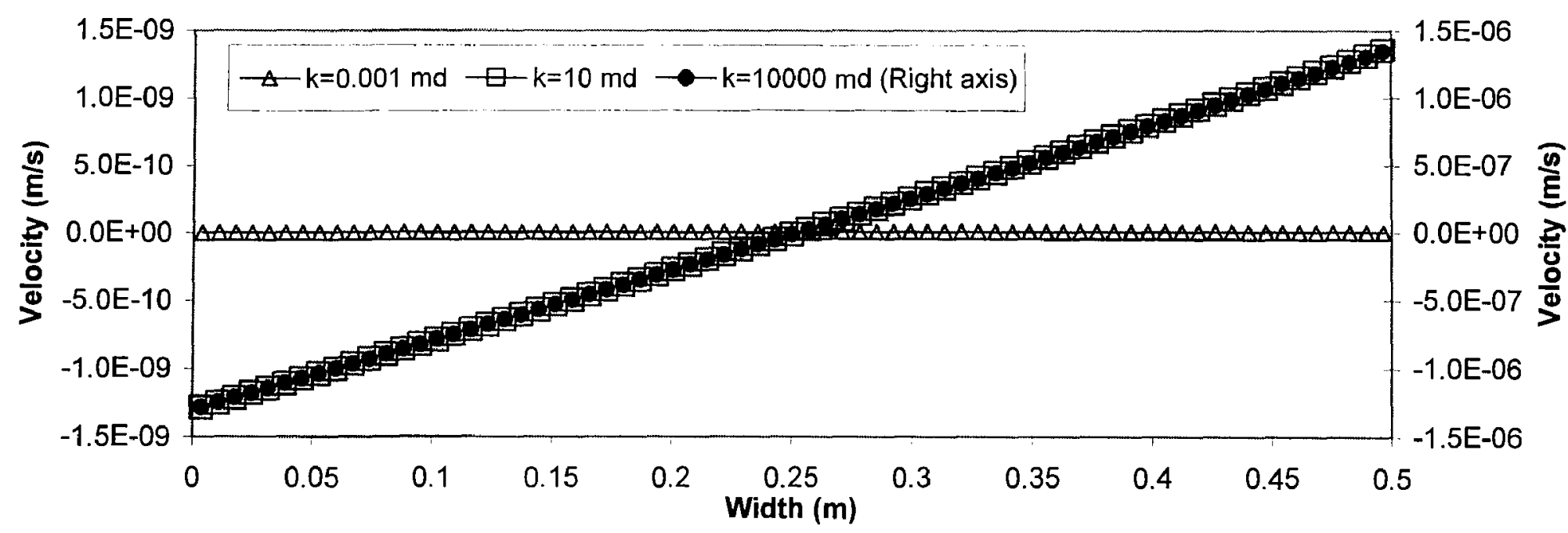

Figure 14: THN-IBB: Velocity along the center of the cavity 


\subsubsection{Comparison of Density distribution along the center of the cavity vertically and horizontally}

The density distribution is also a good indicator of the uniformity or separation of the mixture inside a cavity and the way components of a mixture react to various conditions.

Figure 15 shows the density variation horizontally along the center of the cavity for THN-Dodecane mixture. For low permeability, the density decreases linearly along the width of the cavity. This linear variation also suggests that the Soret effect is dominant and causes the separation in the mixture, which confirms the trend seen in the flow pattern. The heavier component, THN, migrates to the cold wall, while the lighter component, Dodecane, migrates to the hot wall. As the permeability increases, the density variation remains almost linear; however, a slight curve appears in the variation. This is due to the increase in the counter-clockwise flow of the fluid mixture caused by the buoyancy-driven convection. As the permeability increases further, the buoyancydriven convection becomes stronger, and the curve in the variation becomes more apparent. The curvature in the variation indicates that the density gradient in the cavity is diminishing, suggesting that mixing is taking place. The average density for THNDodecane mixture was found to be $803 \mathrm{~kg} / \mathrm{m}^{3}$ approximately. 


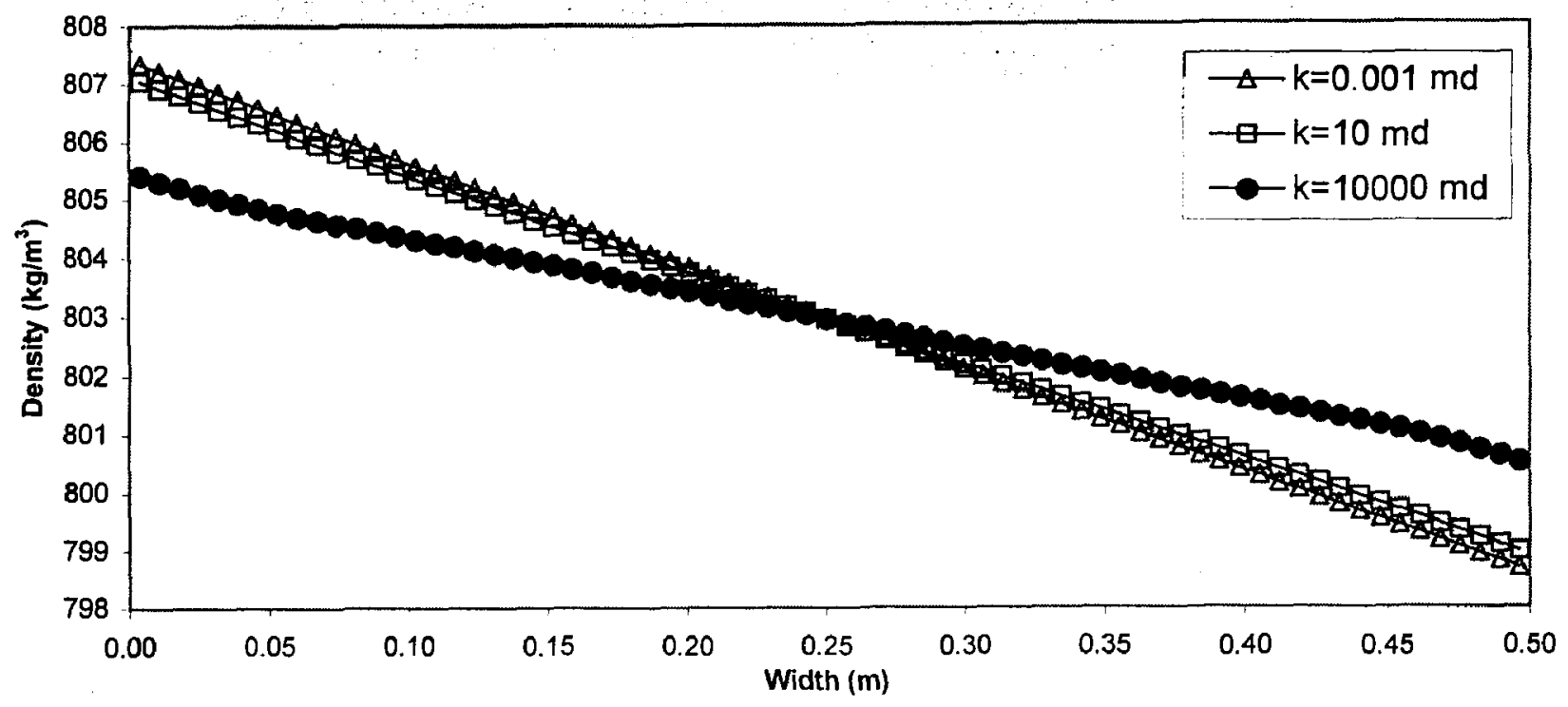

Figure 15: THN-Dodecane: Horizontal density distribution

Figure 16 shows the graph with density variation vertically along the center of the cavity for THN-Dodecane mixture. At lower permeability, a uniform density variation is observed. At such low permeability, the flow is very weak, and the linear horizontal temperature distribution causes a separation in the horizontal direction. As the permeability increases, the vertical density distribution varies linearly. This change comes from the buoyancy-driven convection, which is becoming dominant in the cavity. The heavier component, THN, is being pushed downwards by convection to the bottom of the cavity. Conversely, the lighter component, Dodecane, is being pushed upwards to the top of the cavity. As the permeability increases further, the variation becomes almost uniform again with noticeable changes near the top and bottom of the cavity. The buoyancy-driven convection at higher permeabilities causes the flow to increase in speed; therefore, as the fluid moves rapidly along the top and bottom boundaries of the cavity the variation becomes noticeable, while mixing takes place along the rest of the cavity thereby creating uniformity in the cavity. 


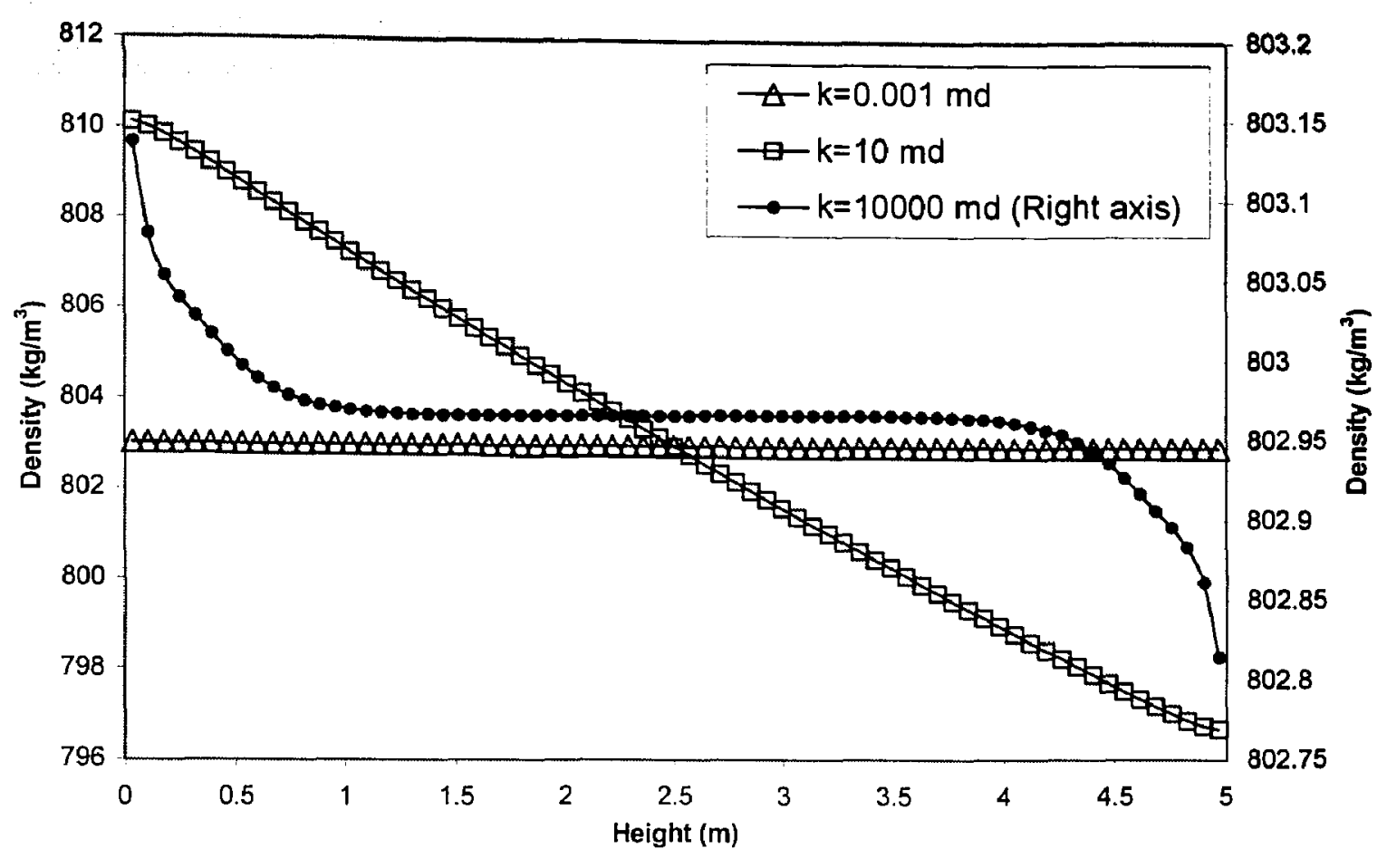

Figure 16: THN-Dodecane: Vertical density distribution

Figure 17 shows the density variation for IBB-Dodecane mixture horizontally along the center of the cavity. It shows a similar pattern to the THN-Dodecane mixture horizontal density variation. It is evident that the density varies linearly at low permeability due to the Soret effect, while the density variation becomes non-linear as the permeabilities increases due to buoyancy convection. The only difference between the two cases is the fact that when the permeability increases, the curvature in the density variation is not as pronounced, as was the case for THN-Dodecane mixture. This is due to the fact that IBB is much more viscous then THN, and hence the fluid mixture cannot flow as rapidly. The average density of IBB-Dodecane mixture is approximately $800 \mathrm{~kg} / \mathrm{m}^{3}$. 


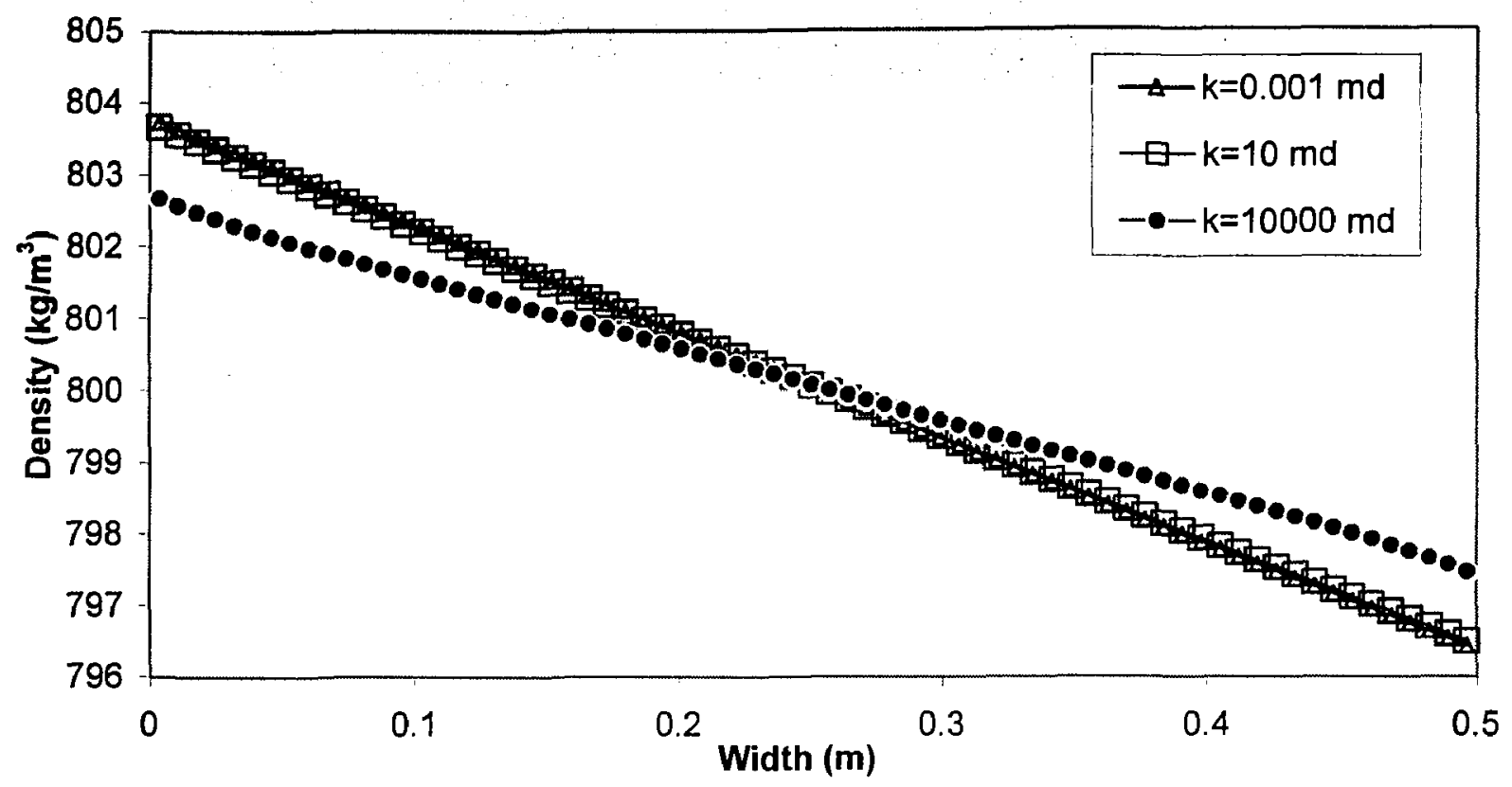

Figure 17: IBB-Dodecane: Horizontal density distribution

Figure 18 shows the density variation for IBB-Dodecane vertically along the center of the cavity. Once again, at lower permeability a uniform density variation is observed, and the variation becomes linear with the increase in the permeability. The change from uniform to linear variation is the result of the convection gaining strength, and causing the IBB to migrate to the bottom of the cavity. As the permeability increases further, the mixture becomes uniform throughout most of the cavity; however, there exists a sharp variation in the density near the top and bottom of the cavity as noticed earlier in the case of THNDodecane mixture. 


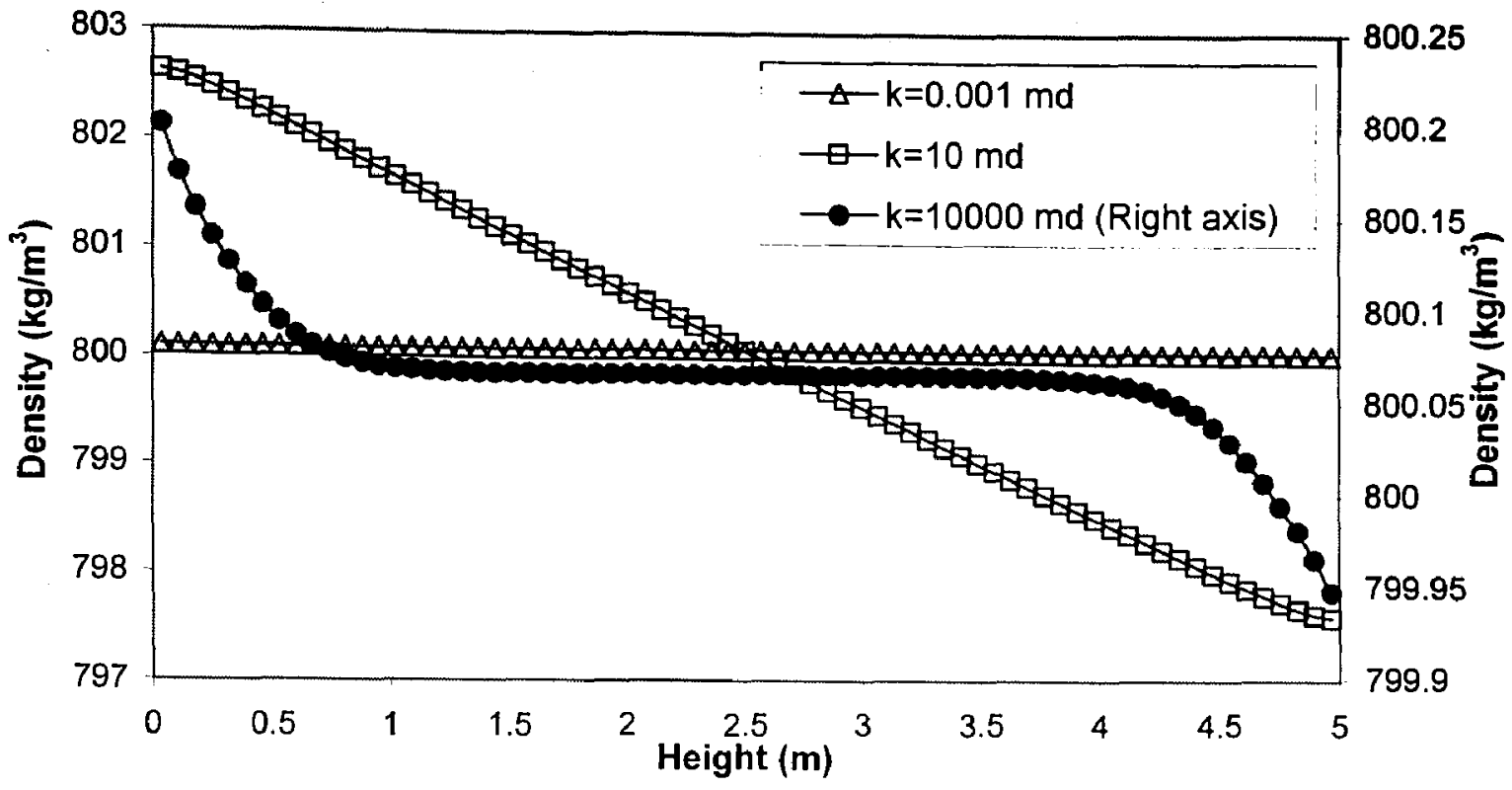

Figure 18: IBB-Dodecane: Vertical density distribution

It can be concluded from the density pattern that at lower permeability, the lateral heating produces a very strong Soret effect in the cavity, which induces a horizontal separation in the cavity. As the permeability increases, the fluid is able to move more readily through out the cavity; hence, the buoyancy-driven convection becomes stronger, and the Soret effect becomes less dominant. As a result of the stronger buoyancy-driven convection and the lateral heating applied to the cavity, the fluid starts to rotate in a counterclockwise direction, in other words, from the hot wall to the cold wall. This rotation causes the heavier component of the mixture to be pushed towards the bottom of the cavity near the cold wall, and the lighter component of the mixture to be pushed upwards nears the hot wall. As the permeability increases further to $10^{4} \mathrm{md}$, which corresponds to a Darcy number of $3.91 \times 10^{-13}$, the buoyancy-driven convection completely takes over inside the cavity, and the Soret effect effectively fades away. Due to the high permeability the fluid has very few impediments and fluid moves almost freely. The fluid velocity inside the cavity therefore increases, equally increases the rotation of the mixture from hot wall to the cold wall. This rotation causes the mixture to mix together.

Figure 19 shows the density variation of the THN-IBB mixture horizontally along the center of the cavity. 


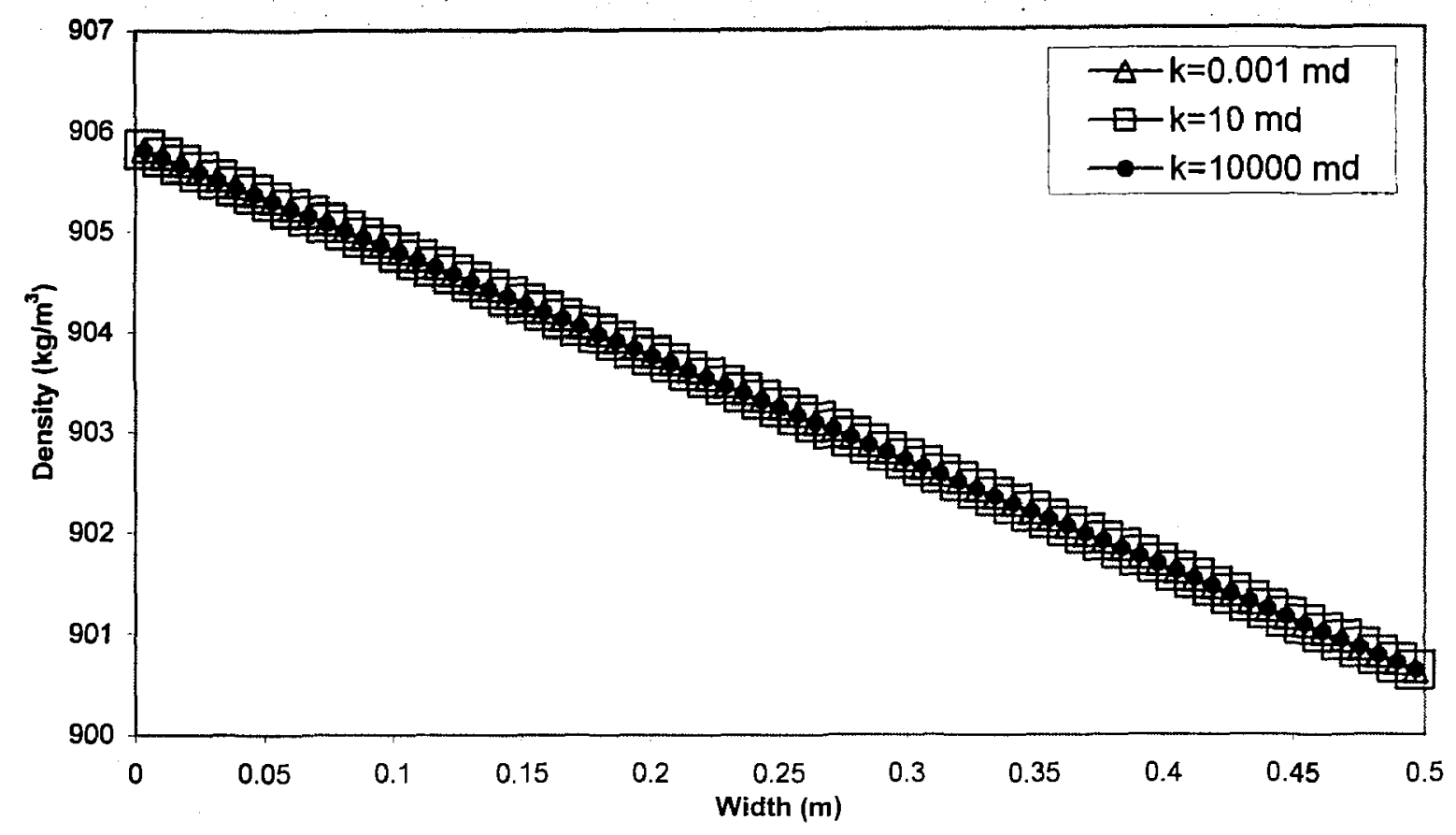

Figure 19: THN-IBB: Horizontal density distribution

Contrary to other binary mixtures of THN-Dodecane and IBB-Dodecane, the density of THN-IBB mixture varies linearly for all ranges of permeabilities. There is little curvature in the density variation as the permeability increases, because the mixture is very viscous. Even at higher permeabilities, where convection is dominant, it is difficult to move the mixture enough to cause mixing, as was the case for the other binary mixtures. Even when the vertical density variation along the center of the cavity is examined, Figure 20, it is evident that variation is modest compared to the other binary mixtures. Even though, by first looking at the graphs, it appears that there is similar pattern to the previous cases, in reality the variations are on the magnitude of $10^{-2} \mathrm{~kg} / \mathrm{m}^{3}$. Therefore, the vertical density variations along the center of cavity have a tendency to remain uniform and can be attributed again to the viscosity and the mixtures ability to mix well together. The average density of THN-IBB mixture is approximately $903.24 \mathrm{~kg} / \mathrm{m}^{3}$. 


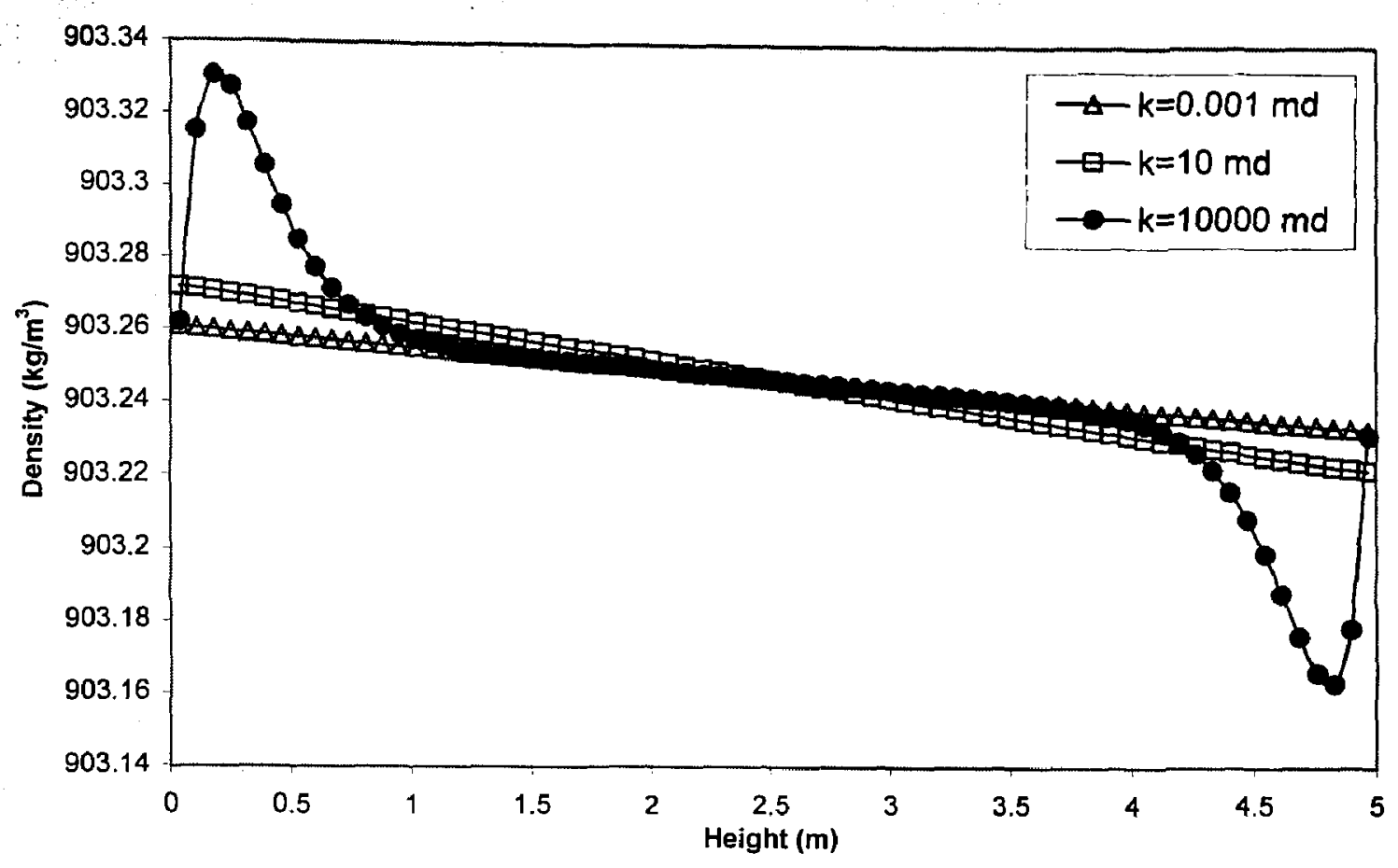

Figure 20: THN-IBB: Vertical density distribution

\subsubsection{Variation of Concentration with permeability}

To analyze the effect of Soret and buoyancy-driven convection in the cavity, the fluid compositional variation is examined. In Figure 21 , it is evident that when the permeability is low and lateral heat is applied to the cavity, a horizontal composition gradient appears because of the Soret effect. The heavier component THN migrates to the cold wall, which agrees with the previous findings when density variation was examined. As the permeability increases, the concentration gradient direction changes, and as the permeability increases further it assumes an angle of almost 45 degrees. The change in direction again points to the fact that the buoyancy-driven convection is increasing in strength, and causing the fluid to flow inside the cavity. As the permeability continues to increase, the concentration gradient becomes almost vertical with heavier THN accumulating near the bottom of the cavity, which once again agrees with the earlier findings. At high permeability values, the concentration gradient no longer exists, and a clear mixing pattern is visible, with flow moving in a counter-clockwise direction. 
A similar pattern is seen in the binary mixture of IBB-Dodecane, in Figure 22, and THNIBB, Figure 23. The concentration gradient is horizontal at low permeability then changes direction to almost 45 degrees as permeability increases. The gradient is caused by the contribution of both the Soret effect and buoyancy-driven convection. As the permeability increases, the concentration gradient diminishes and a mixing pattern is visible, due to the buoyancy-driven convection dominating inside the cavity. 
Figure 21: THN-Dodecane: Concentration contours for permeability ranging from $0.001 \mathrm{md}$ to $10,000 \mathrm{md}$

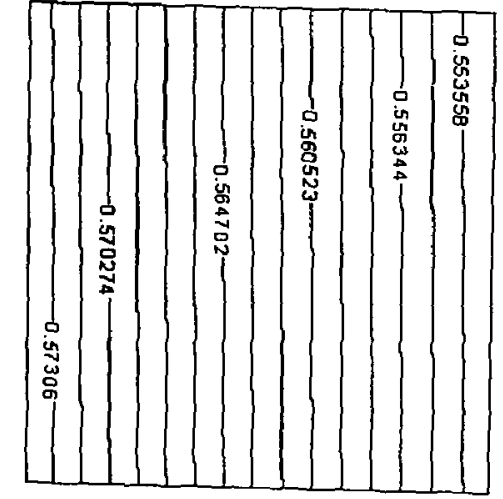

a). $k=0.001 \mathrm{md}$

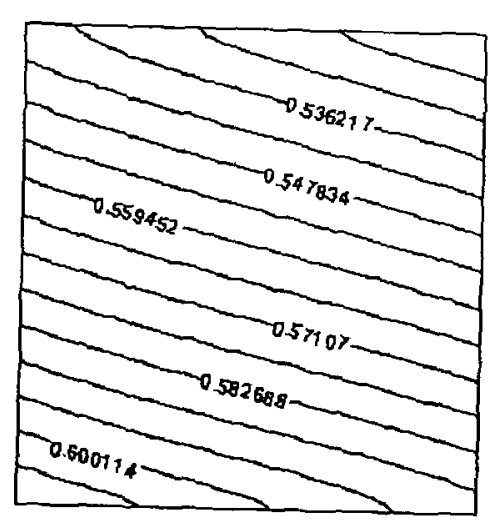

e). $k=10 \mathrm{md}$

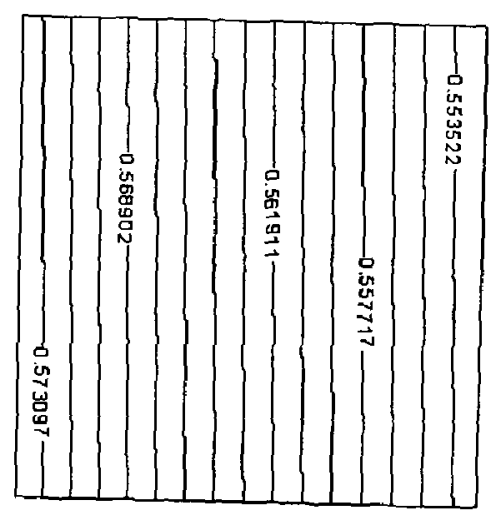

b). $k=0.0 \mathrm{Imd}$

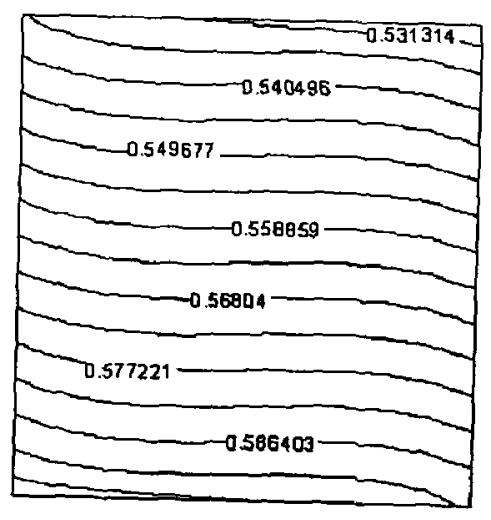

f). $k=100 \mathrm{md}$

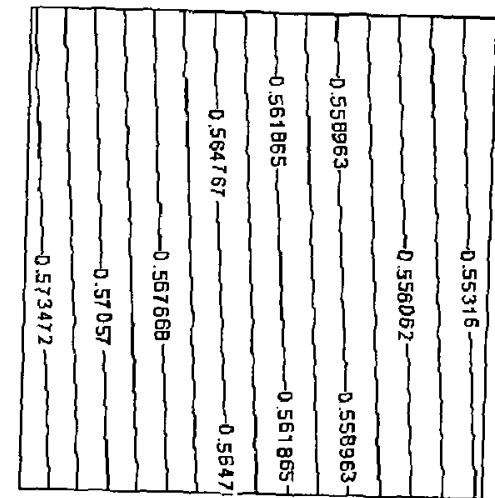

c). $\mathrm{k}=0.1 \mathrm{md}$

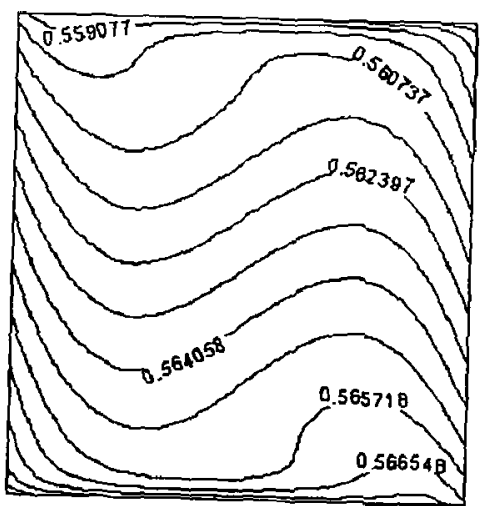

g). $k=1000 \mathrm{md}$

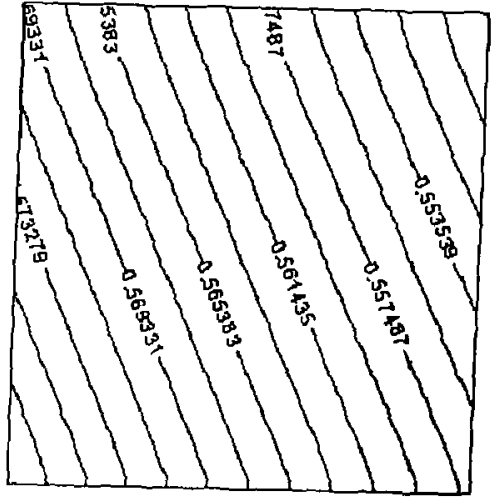

d). $k=1 \mathrm{md}$

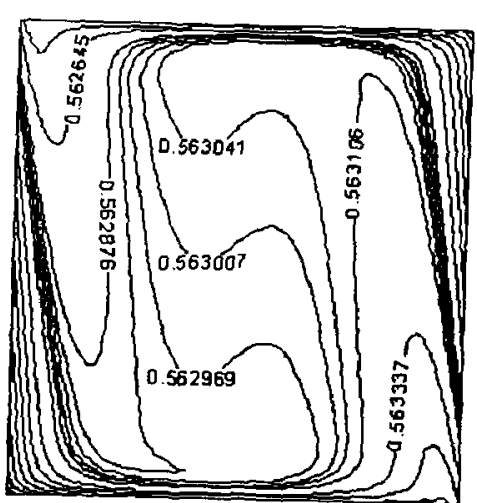

h). $k=10000 \mathrm{md}$ 
Figure 22: IBB-Dodecane: Concentration contours

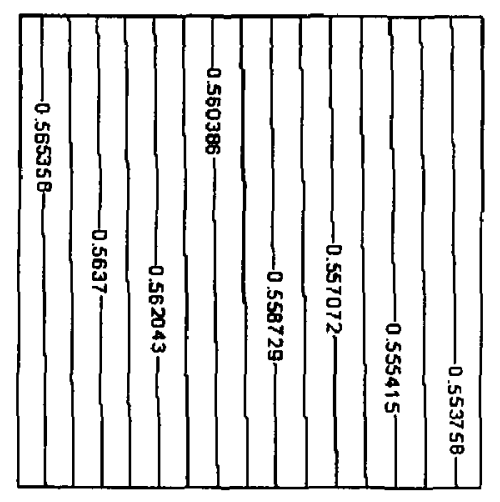

a). $\mathrm{k}=0.001 \mathrm{md}$

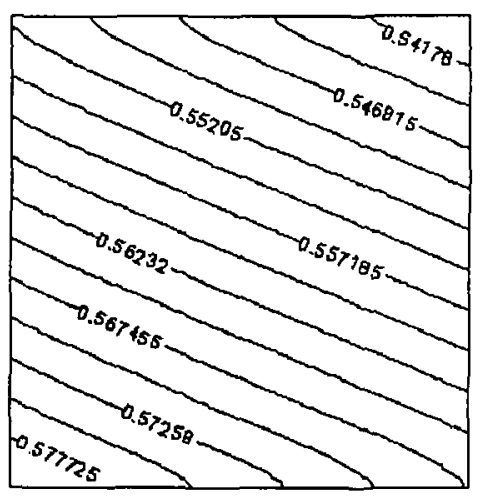

b). $k=10 \mathrm{md}$

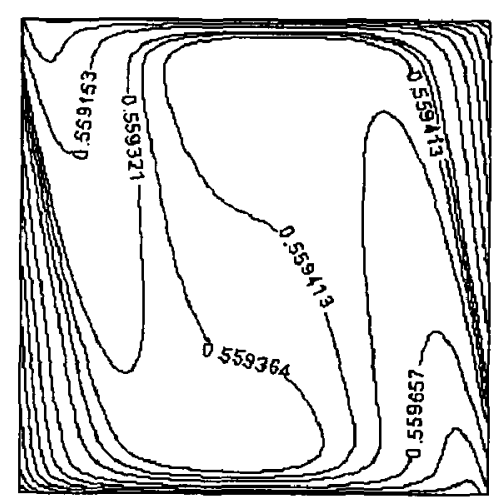

c). $\mathrm{k}=10000 \mathrm{md}$

Figure 23: THN-IBB: Concentration contours

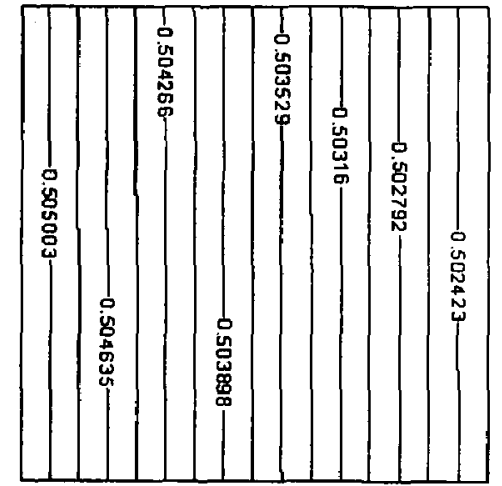

a). $k=0.001 \mathrm{md}$

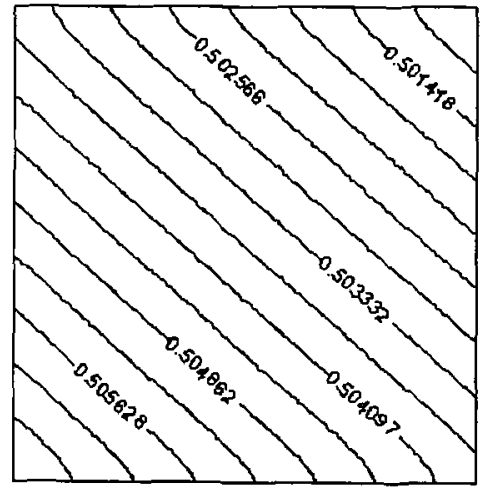

b). $\mathrm{k}=10 \mathrm{md}$

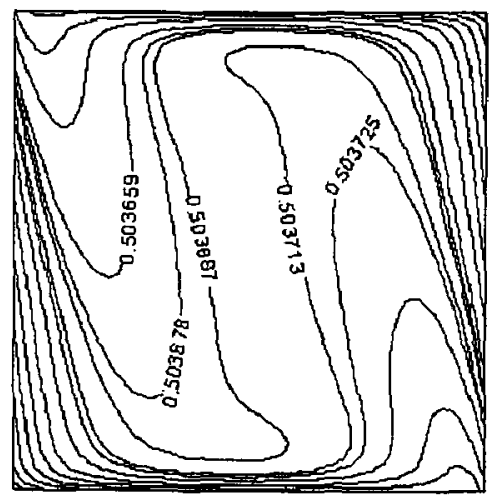

c). $\mathrm{k}=10000 \mathrm{md}$ 


\subsubsection{Separation Ratio}

It is evident that the permeability has a direct effect on the separation of the components in the mixture. At lower permeabilities, the Soret effect has a significant impact on the mixture, while at higher permeabilities, convection become a major factor. One method of examining the effect of permeability further is by means of a variable known as the separation ratio $(\mathrm{q})$. The separation ratio can be defined as follows:

$$
q=\frac{C_{c} /\left(1-C_{c}\right)}{C_{l 1} /\left(1-C_{h}\right)}
$$

where, $C_{c}$ represents the concentration of a particular component at the cold wall, and $C_{h}$ represents the concentration of the same component at the hot wall. A variation in the separation ratio as a function of the permeability is shown in Figure 24. The separation ratio variation with permeability indicates three noticeable regimes. The first regime is between $0.001 \mathrm{md}$ and $0.1 \mathrm{md}$, where the separation ratio remains constant and maintains a value below 1 . This regime is caused by the strong thermal diffusion, while the convection effect is negligible. Since the value of separation ratio is less then 1 , it is further indicative of the fact that a concentration gradient exists horizontally along the width of the cavity, with a higher concentration of the heavier component on the cold wall, and a lighter component on the hot wall. Therefore, a value of the separation ratio that is closer to the value of one $(\mathrm{q}=1)$ specifies that the Soret effect is weak, and a value further from the value of one $(q=1)$ indicates that the Soret effect is stronger and causing perceptible separation in the cavity. Among the three binary mixtures, the Soret effect is strongest at lower permeability in the THN-Dodecane mixture, while it is weakest in the THN-IBB.

The second regime is between $0.1 \mathrm{md}$ and $100 \mathrm{md}$. In this regime, the separation ratio value increases and reaches a peak value. This peak value indicates the permeability 
value where the maximum separation of components occurs. The increase in the separation ratio also indicates the change in orientation in the concentration gradient. The concentration gradient, with increasing permeability, slowly becomes horizontal. The maximum separation occurs when the heavier component accumulates on the bottom of the cavity near the cold wall, and the lighter component accumulates on the top of the cavity near the hot wall. This regime also indicates that separation is caused by the contribution of both the Soret effect and buoyancy-driven convection. In Figure 24, it is evident that the THN-Dodecane mixture has the highest value of Separation ratio, while THN-IBB mixture has the lowest value.

The third regime is between $100 \mathrm{md}$ and $10,000 \mathrm{md}$. In this regime, the separation ratio decreases rapidly and reaches a value close to 1 . The reason the separation ratio decreases is because the buoyancy-driven convection is becoming dominant and causes the mixture to mix. As the permeability reaches $10^{4} \mathrm{md}$, the mixture is well mixed and there is little or no separation. Therefore, in this regime, the convection becomes dominant and the Soret effect is suppressed. 


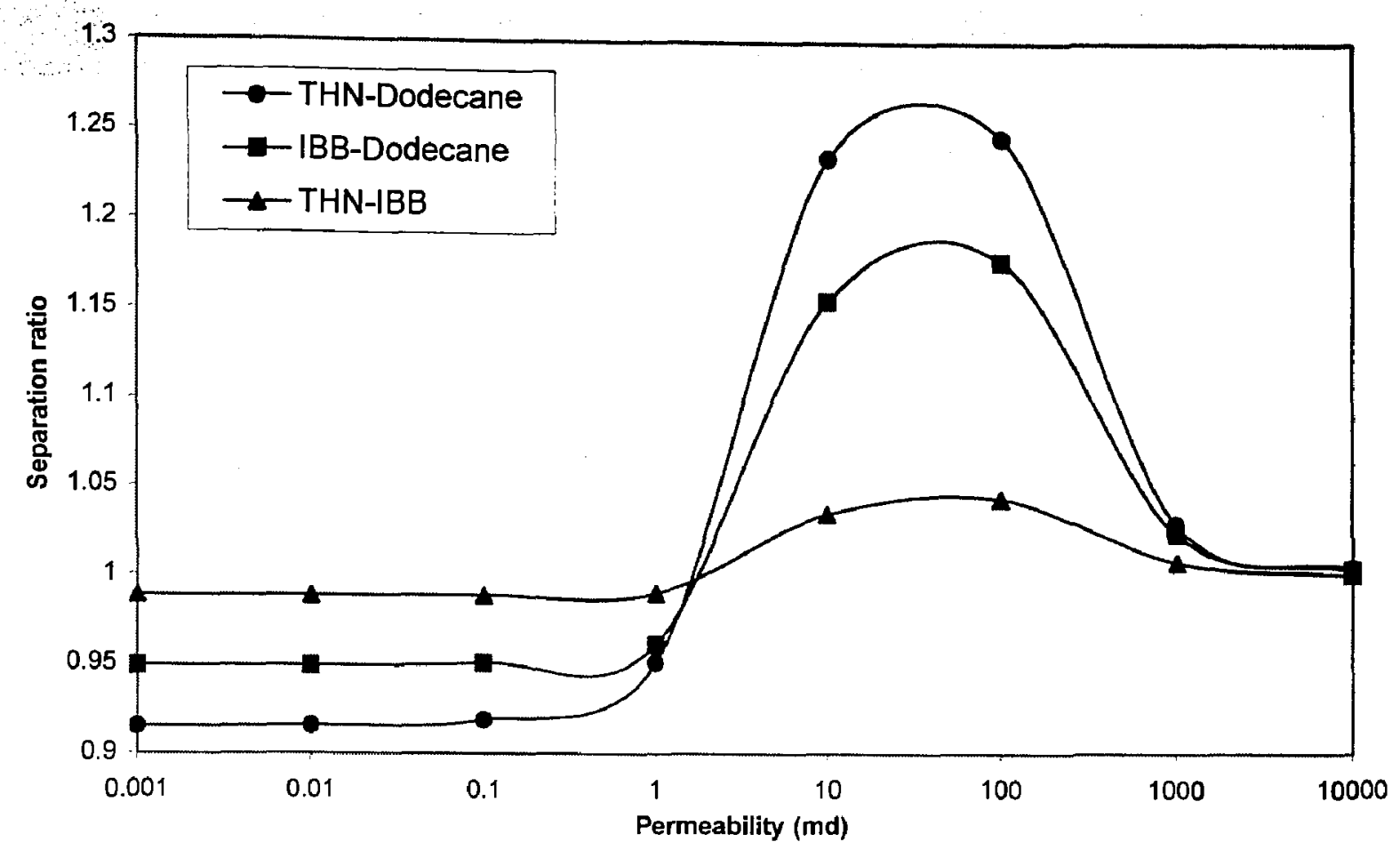

Figure 24: Separation ratio as a function of the permeability

Benano-Melly et al. [14] showed that a maximum value for the separation ratio exists for a permeability given by the following expression:

$$
\kappa=\frac{\mu \mathrm{D}^{\mathrm{M}} \varphi \sqrt{120}}{\mathrm{~g} \beta_{\mathrm{T}} \Delta \mathrm{TH} \rho}
$$

Table 7 shows the maximum separation according to the analytical expression and the value of maximum separation obtained by the numerical simulation.

Table 7: Maximum Separation obtained by Analytical expression and Numerical Simulation

\begin{tabular}{lcc}
\hline & $\begin{array}{c}\text { Analytical Maximum Separation } \\
\text { Ratio [md] }\end{array}$ & $\begin{array}{c}\text { Numerical Maximum Separation } \\
\text { Ratio [md] }\end{array}$ \\
\hline THN-Dodecane & 21.69 & 25 \\
IBB-Dodecane & 34.50 & 39 \\
THN-IBB & 44.28 & 50 \\
\hline
\end{tabular}


The discrepancy between the values obtained by the analytical expression and by numerical modeling is due to the fact that solutal buoyancy is included in the numerical simulation; in addition, both thermal and molecular diffusion coefficients are functions of temperature, fluid mixtures, and pressure.

Peaking of the separation ratio in the second regime might be explained by the fluid and thermal characteristic times, as shown in the Figure 25 for the THN-Dodecane. The characteristic time for thermal diffusion $\tau_{\text {th }}$ is found to be constant at $1.17 \times 10^{8}$ seconds, while the flow characteristic time $\tau_{\mathrm{f}}$ decreases monotonically from $1 \times 10^{10}$ seconds to $3.69 \times 10^{6}$ seconds, as the permeability increases. The point of intersection of the fluid characteristic time and thermal characteristic time occurs at approximately $25 \mathrm{md}$, which corresponds to the value of permeability where the separation ratio is observed to be at its maximum value. Consequently, the point of intersection also indicates that beyond this point the fluid characteristic time will be less than the thermal characteristic time, hence indicating that the buoyancy-driven convection becomes the dominant force in the cavity. 


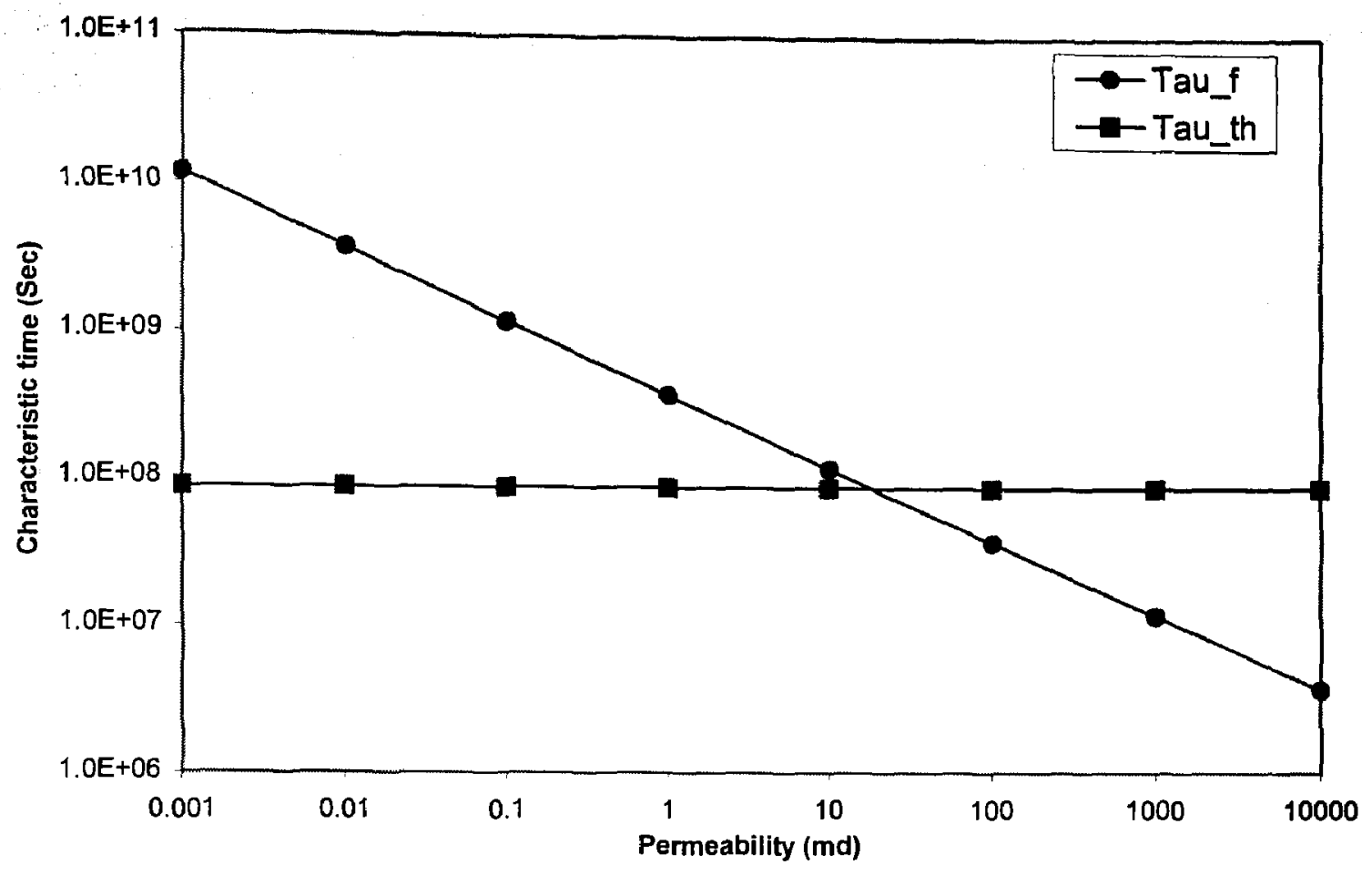

Figure 25: Characteristic times as a function of the permeability

Another way to study this effect is by plotting the ratio of solutal and thermal Rayleigh numbers as a function of the permeability, as shown in Figure 26. The ratio can be defined as follows:

$$
\frac{\operatorname{Ra}_{c}}{\operatorname{Ra}_{\mathrm{T}}}=\operatorname{Le} \frac{\beta_{\mathrm{c}} \Delta \mathrm{c}}{\beta_{\mathrm{T}} \Delta \mathrm{T}}
$$

where, Le is the Lewis number. The Rayleigh number is defined as the product of the Grashof number, which describes the relationship between buoyancy and inertia within a fluid, and the Prandtl number. Figure 26 illustrates that the concentration difference remains uniform for permeability between $0.001 \mathrm{md}$ and $0.1 \mathrm{md}$, because the ratio of Rayleigh number remains constant. The concentration difference increases from $0.1 \mathrm{md}$ until $100 \mathrm{md}$, and then decreases from $100 \mathrm{md}$ to $10,000 \mathrm{md}$. It can be realized that, for permeability values between $0.001 \mathrm{md}$ and $0.1 \mathrm{md}$, as the thermal Rayleigh number increases linearly, the solutal Rayleigh number begins to increases linearly as well, indicating the dominance of the Soret effect. Between $0.1 \mathrm{md}$ and $100 \mathrm{md}$, a slight 
increase and then a peak around $25 \mathrm{md}$ in the ratio of Rayleigh numbers variation indicates that the Soret effect and buoyancy-driven convection are both active in the cavity. Beyond a permeability of $100 \mathrm{md}$, there is a reduction in the solutal Rayleigh number; however, the thermal Rayleigh number continues to increase linearly indicating the dominance of buoyancy-driven convection.

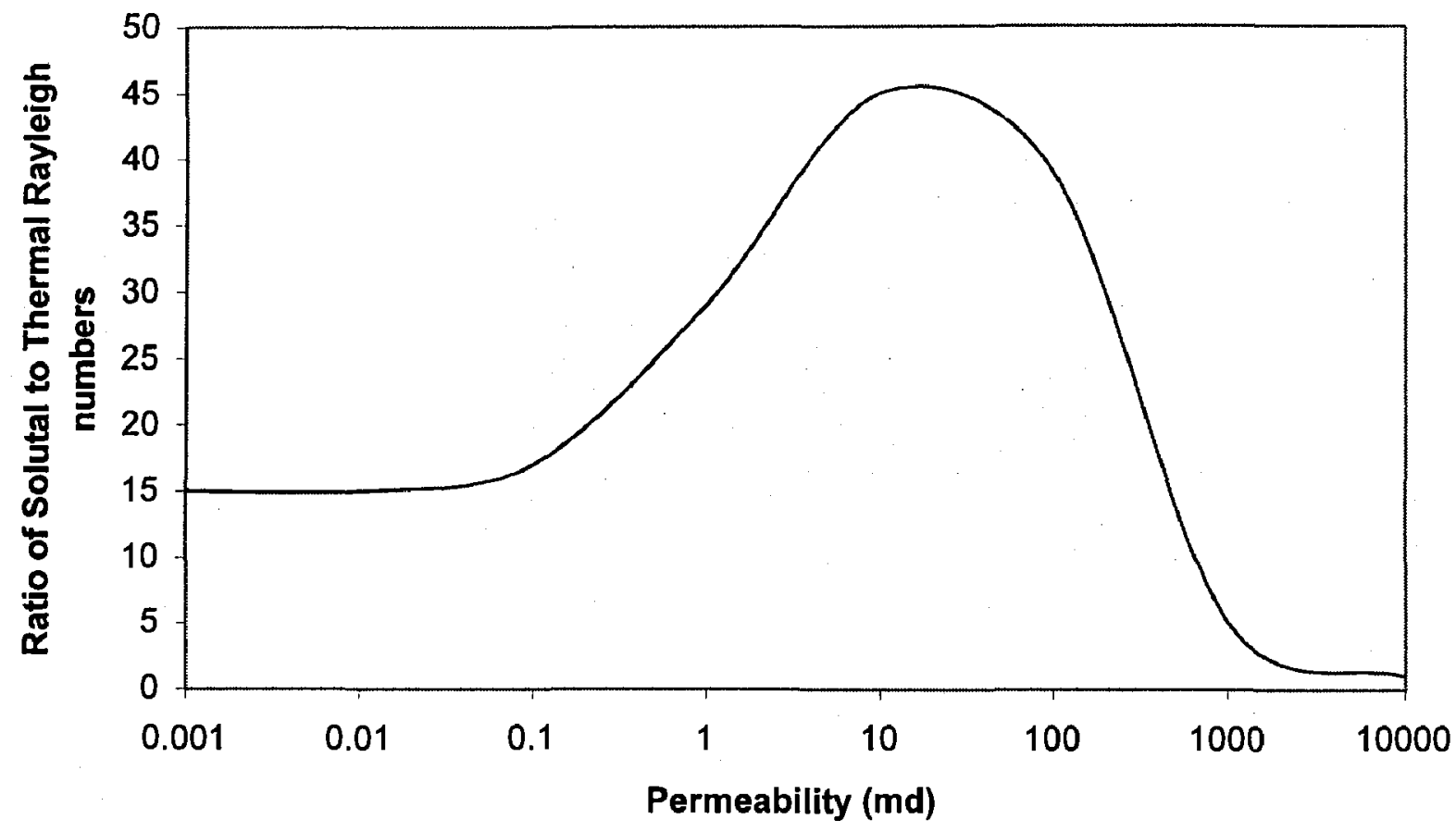

Figure 26: Variation of the Rayleigh number

\subsection{SUMMARY}

This chapter investigates the interaction between thermal diffusion and buoyancy driven convection in a single-phase hydrocarbon binary mixture system. Three binary mixtures of THN-Dodecane, IBB-Dodecane, and THN-IBB each with 50\% mass fraction, were used in the simulation. The model is based on non-equilibrium thermodynamics theory, and the diffusion coefficients are evaluated with time and space dependent fluid properties and compositions. In the analysis, the flow characteristics, the velocity in the cavity, the component concentration gradients, and the separation ratio have been used to 
investigate the behaviour of the thermal diffusion and buoyancy-driven convection. The analysis reveals a consistent behaviour between the three binary mixtures. It also reveals that the THN-Dodecane mixture separates the most, while THN-IBB separates the least inside the cavity. The main conclusion from this analysis is that the convection effect on thermal diffusion in a hydrocarbon binary system could be explained in terms of the characteristic times. When the characteristic time of the convection flow, $\tau_{\mathrm{f}}$, is greater than the characteristic time of thermal diffusion, $\tau_{\text {tl }}$, then the thermal diffusion effect is the dominant force for the component separation in the cavity. In other words, the convection transfers information slower than thermal diffusion, then it contributes to the Soret effect becoming stronger. The maximum separation is reached when the two characteristic times are equal to one another. When the characteristic time of the convection flow is less than the characteristic time of thermal diffusion, the buoyancy driven convection becomes dominant. Therefore, when the convection transfers information faster than the thermal diffusion, then the contribution of buoyancy driven convection becomes dominant, and the thermal diffusion effect diminishes. 


\section{CHAPTER 5: TWO DIMENSIONAL SIMULATION OF THERMAL DIFFUSION IN A HETEROGENEOUS POROUS MEIDA}

\subsection{INTRODUCTION}

A reservoir is a porous and permeable geological unit or set of units that may hold underground water in aquifers or hydrocarbons in oil and gas reservoirs. Even at the simplest levels, the reservoir analysis requires an assessment of their porosity and their permeability. In other words, it requires the analysis of the volume of the in situ hydrocarbon, and the measure of how easily hydrocarbons flow through or out of them. Naturally occurring reservoirs are found at various depths and in various sizes, and tend to contain fractures.

The research on fluid flow in fractured porous media has been continuing since the 1960's. Barrenblatt et al. [42], along with Warrant and Root [43], introduced the concept of multi-porosity to analyze fractures in reservoirs. They presented an idealized model as a set of highly interconnected fractures, which is supplied by fluids from numerous small matrix blocks around them. Kazemi et al. [44] were the first to incorporate the multiporosity concept into a numerical model, with application to fluid flow on a large scale. They also extended the multi-porosity model to a multi-porosity/multi-permeability model, where the simulation of fractured reservoirs involves discretization of the solution domain into two continua, one domain representing the primary matrix, and a secondary domain representing fractured formulations. In other words, imaging the reservoir as a heterogeneous porous media. There are a large number of publications that study the effect of heterogeneity in a porous media, such as Ingham et al, $[2,3]$, where the viscosity effect on the Darcy Law from a mathematical approached is discussed. Storesletten et al. [45] investigated the impact of heterogeneous permeability on convective flow and detailed the convection flow characteristics with respect to different permeability ratios. Saghir and Islam [46] studied the effect of convection in a multi-porosity/multipermeability porous cavity using salt and sugar water. However, these publications do not take into account the thermal diffusion process in heterogeneous porous media. Jiang 
et al. [22] investigated the effect of thermodiffusion in a multi-permeability cavity that was laterally heated. However, the research did not incorporate the multi-porosity and only investigated horizontal fractures.

This chapter investigates numerically the effects of both horizontal and vertical fractures, using a multi-porosity/multi-permeability model on the thermal diffusion and buoyancydriven convection in a laterally heated vertical porous cavity filled with a THN-Dodecane binary mixture. Furthermore, the size of the horizontal and vertical fractures is varied to investigate the influence of fracture size on the thermal diffusion and buoyancy-driven convection.

\subsection{THE NUMERICAL MODEL AND VALIDATION}

A vertical cavity with an aspect ratio of 10 with the same boundary conditions as the previous homogeneous porous media case is utilized (see Figure 7). The binary mixture of THN-Dodecane, each component at 50\% mass fraction, and under atmospheric pressure was used. Two main cases were simulated, the first representing the horizontal fracture and the second representing the vertical fracture, as shown in Figure 27. The two main cases were further analyzed by varying the size of the fracture. 

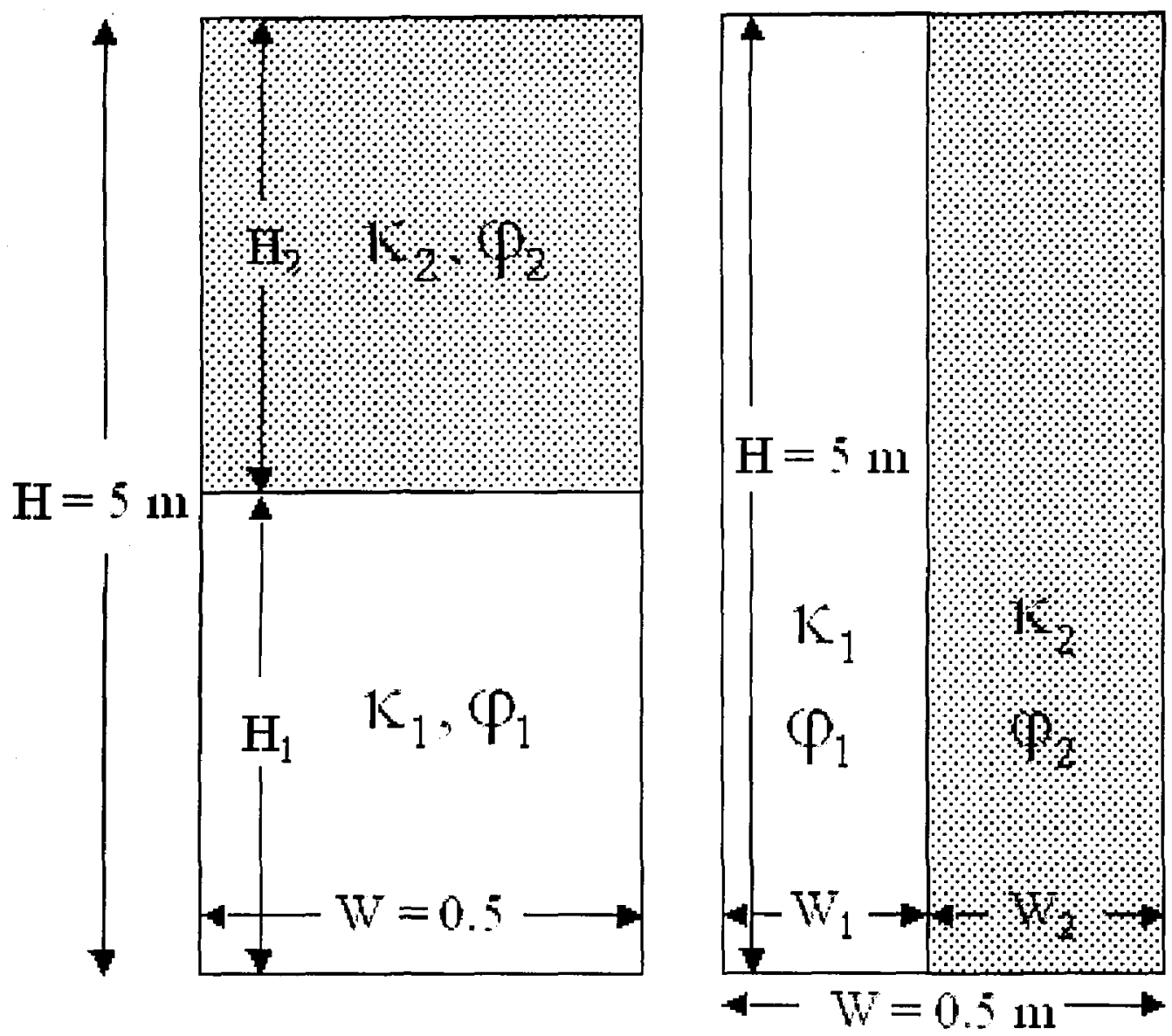

Figure 27: Two-dimensional heterogeneous porous media cavity; the left represents the horizontal fracture case, and the right represents the vertical fracture case

The permeability $\left(\kappa_{1}\right)$ and porosity $\left(\varphi_{1}\right)$ in the primary domain are $0.01 \mathrm{md}$ and 0.20 respectively, while the permeability $\left(\kappa_{2}\right)$ and porosity $\left(\varphi_{2}\right)$ in the fracture are $1 \mathrm{md}$ and 0.40 respectively.

For the horizontal fracture case, three cases were analyzed, first with the fracture size $\left(\mathrm{H}_{2}\right)$ equal to $2.5 \mathrm{~m}$, second with the fracture size $\left(\mathrm{H}_{2}\right)$ equal to $1.5 \mathrm{~m}$, and third with the fracture size $\left(\mathrm{H}_{2}\right)$ equal to $0.5 \mathrm{~m}$. For the vertical fracture case, three cases were also analyzed, first with the fracture size $\left(\mathrm{W}_{2}\right)$ equal to $0.25 \mathrm{~m}$, second with the fracture size $\left(\mathrm{W}_{2}\right)$ equal to $0.15 \mathrm{~m}$, and third with the fracture size $\left(\mathrm{W}_{2}\right)$ equal to $0.05 \mathrm{~m}$.

In order to verify the numerical simulation is accurate when fractures are introduced, a test case was simulated. First, the previous homogeneous case was simulated and 
streamlines plotted. Then a horizontal fracture was introduced in the geometry file; however, the porosity and permeability in both domains were kept the same as the homogeneous case. In other words, a physical fracture is present in the cavity; however, the properties in both domains are the same. The streamlines were plotted again, and it was noticed that the values were exactly the same between the two cases, therefore verifying the fact that numerical simulation is unaffected by the presence of the fracture. Figure 28 shows streamlines for the un-fractured and fractured cases.

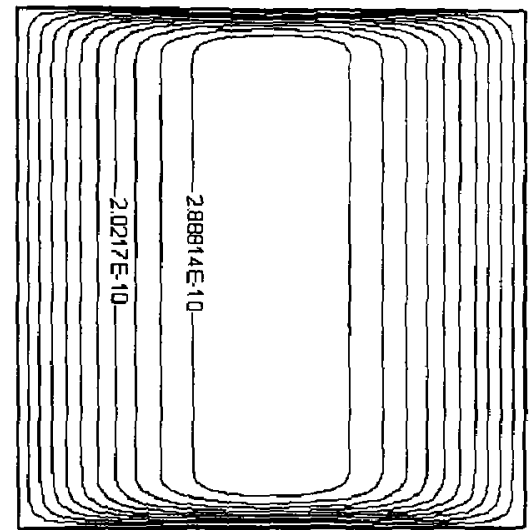

a). Un-fractured case

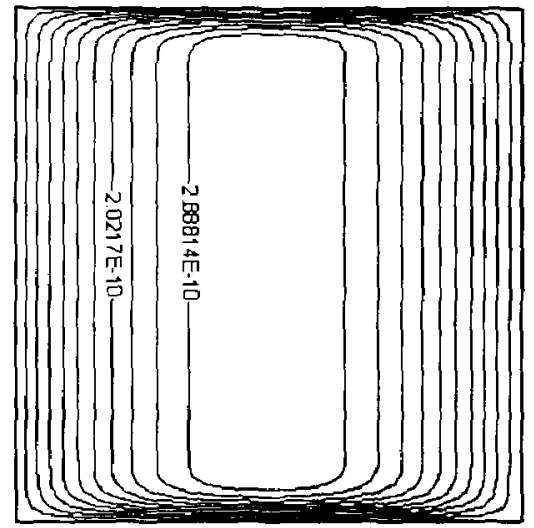

b). Fractured case

Figure 28: Streamline contours for un-fractured and fractured case

\subsection{RESULTS AND DISCUSSION}

The present objective is to not only investigate the interaction between thermal diffusion and buoyancy-driven convection but also to analyze the effect of fracture on these phenomenons. In order to make the analysis more realistic, various sizes of fractures are utilized. The numerical simulation will be used to analyze the flow characteristics, the velocity in the cavity, and the concentration gradients in the cavity. The Soret coefficient is also examined for both vertical and horizontal cases.

\subsubsection{Flow patterns}

The streamlines indicating the flow behaviour of THN-Dodecane binary mixture inside a vertical cavity with a horizontal crack is depicted in Figure 29. 


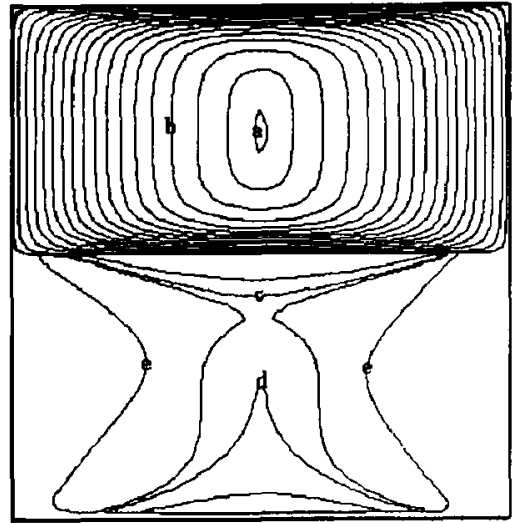

a). Fracture size $=2.5 \mathrm{~m}$

$$
\begin{gathered}
a=1.635 \times 10^{-8} \\
b=1.431 \times 10^{-8} \\
c=3.277 \times 10^{-10} \\
d=3.276 \times 10^{-10} \\
e=2.375 \times 10^{-10} \\
\Delta \psi=8.055 \times 10^{-10}
\end{gathered}
$$

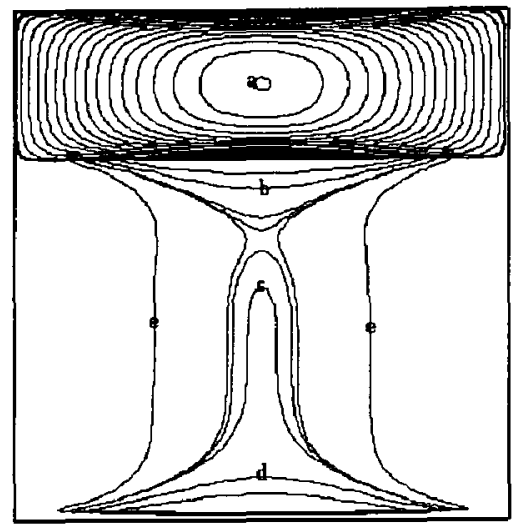

b). Fracture size $=1.5 \mathrm{~m}$

$$
\begin{gathered}
a=1.606 \times 10^{-8} \\
b=3.688 \times 10^{-10} \\
c=3.293 \times 10^{-10} \\
d=3.688 \times 10^{-10} \\
e=2.393 \times 10^{-10} \\
\Delta \psi=7.189 \times 10^{-10}
\end{gathered}
$$

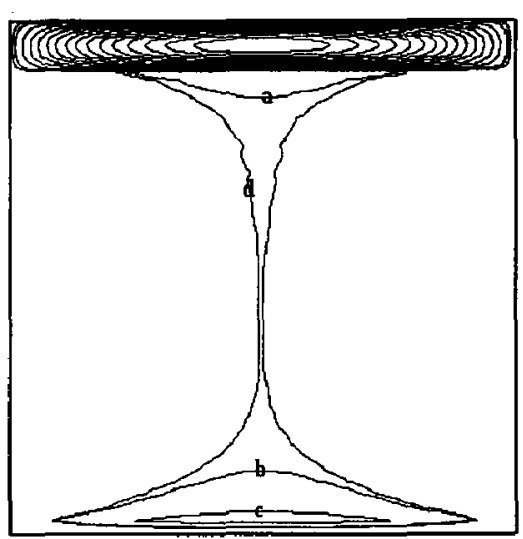

c). Fracture size $=0.5 \mathrm{~m}$

$$
\begin{aligned}
a & =8.908 \times 10^{-9} \\
b & =3.583 \times 10^{-10} \\
c & =3.344 \times 10^{-10} \\
d & =4.987 \times 10^{-10} \\
\Delta \psi & =5.047 \times 10^{-10}
\end{aligned}
$$

Figure 29: Streamline contours for horizontal fracture

It is noticed that for the case when the horizontal crack is $2.5 \mathrm{~m}$, or when half the height of the cavity is fractured, a really strong and a well-defined convective cell is obvious in the fracture. This convective cell is created by buoyancy-driven convection because of the high permeability. The streamline pattern also indicates that the counter-clockwise flow in the fractured cavity seeps out close to the cold wall and continues to penetrate the primary domain until the center of the cavity is reached. The flow then re-enters the fractured cavity after this point. This creates a thick plume of fluid in the center of the cavity. Therefore, the fluid goes down the primary domain until the bottom of the cavity is reached; there it starts to accumulate. Then, the accumulated fluid from the bottom of the cavity goes up along the primary domain until it reaches the fracture and it is then recirculated by the convective cell. This process happens simultaneously, and effectively creating two artificial convective cells on either side of the central plume. The evidence of the artificial convective cells in the primary domain is clear by examining the velocity along the height of the cavity at quarter of the width of the cavity, which is shown in Figure 30. It indicates that the velocity is negative in the primary domain until the fracture is reached; the velocity then becomes positive. It also indicates that when the 
fracture size is half the size of the cavity, the velocity varies linearly inside the cavity. The reason the flow is moving slowly in the primary domain is due to the fact that the permeability is low, so buoyancy-driven convection is not very strong; however, the Soret effect is dominant. Therefore, in the cavity the effect of both the Soret effect and buoyancy-driven convection are present. While the Soret effect dominates the lower domain, the buoyancy-driven convection dominates the upper domain.

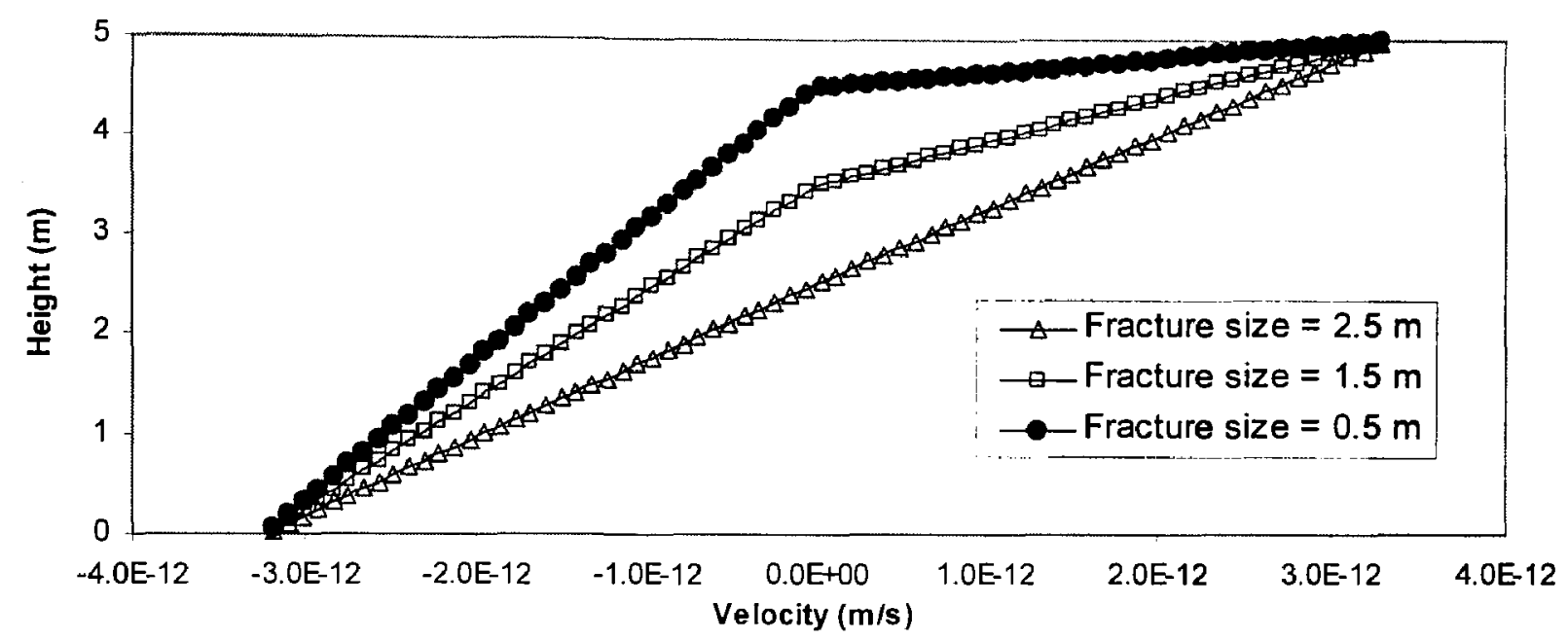

Figure 30: Horizontal fracture: Velocity vertically inside the cavity

A similar pattern is observed when the fracture size is decreased to $1.5 \mathrm{~m}$. The streamlines again indicate a convective cell in the fracture and a plume of fluid along the center of the cavity. The center plume is, however, not as thick as the case when half of the cavity was fractured. This is due to the fact that fluid has to travel a longer distance down the primary domain to the bottom of the cavity and back up to the fracture, therefore elongating the plume and making it appear narrower. The velocity profile along the height of the cavity at quarter of the width of the cavity indicates, once again, two artificial convective cells on either sides of the central plume in the lower domain that is rotating in the opposite direction as the top convective cell. Furthermore, the velocity increases linearly along the height of the cavity and, when it reaches the fracture, the slope of the line decreases, indicating faster flow. Once again, the Soret effect is dominant in the primary domain and buoyancy-driven convection is stronger in the fractured domain. 
A similar pattern is observed when the fracture size is decreased to $0.5 \mathrm{~m}$. The streamlines again indicate the convective cell in the fracture, and an even narrower plume of fluid is present in the center of the cavity. The velocity profile indicates a linear variation throughout the primary domain with a slope decreasing when the fractured domain is reached, indicating faster flow in the upper domain. Furthermore, since the fracture is so small in size, the Soret effect has more influence in the entire cavity.

The streamlines indicating the flow behaviour of the THN-Dodecane binary mixture inside a vertical cavity with a vertical crack is depicted in Figure 31.

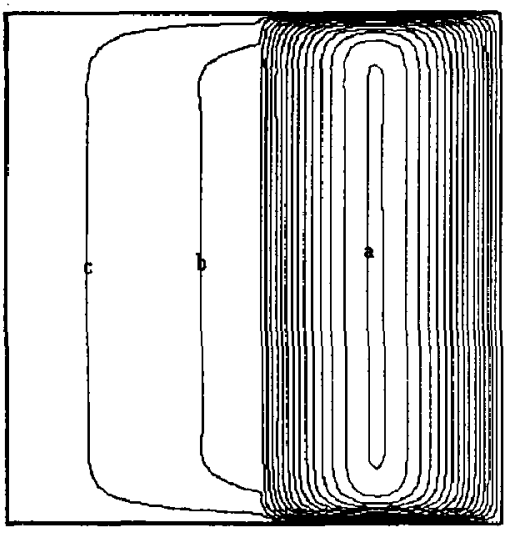

a). Fracture size $=0.25 \mathrm{~m}$

$$
\begin{gathered}
a=4.280 \times 10^{-9} \\
b=5.369 \times 10^{-10} \\
c=2.684 \times 10^{-10} \\
\Delta \psi=2.674 \times 10^{-10}
\end{gathered}
$$

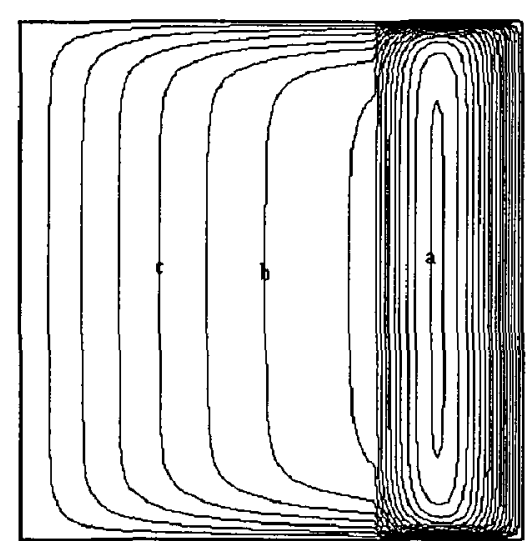

b). Fracture size $=0.15 \mathrm{~m}$

$$
\begin{gathered}
\mathrm{a}=1.902 \times 10^{-9} \\
\mathrm{~b}=7.168 \times 10^{-10} \\
\mathrm{c}=4.779 \times 10^{-10} \\
\Delta \psi=1.188 \times 10^{-10}
\end{gathered}
$$

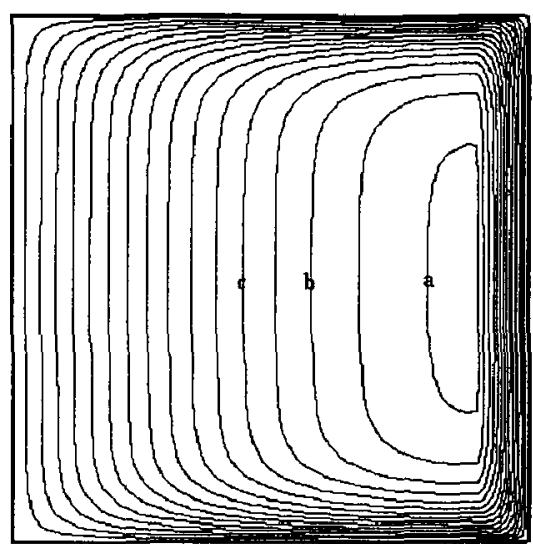

c). Fracture size $=0.05 \mathrm{~m}$ $a=9.824 \times 10^{-10}$

$\mathrm{b}=8.691 \times 10^{-10}$

$\mathrm{c}=6.829 \times 10^{-10}$ $\Delta \psi=6.136 \times 10^{-11}$

Figure 31: Streamline contours for horizontal fracture

It is noticed that for the case when the vertical crack is $0.25 \mathrm{~m}$, or half of the width of the cavity is fractured, a convective cell is observed in the fracture. The convective cell is rotating in the counter-clock wise direction; and is caused by the buoyancy-driven convection in the fracture. The buoyancy-driven convection is stronger in the fracture because the permeability and porosity are high, and the fluid can move easily in the 
fracture. It is also noticed that some of the flow is penetrating the primary domain along the top of the cavity and then re-enters the fracture near the bottom. When the velocity along the width of the cavity at quarter of the height is examined, it reveals that the velocity in the primary domain is very slow, as shown in Figure 32. This is due to the lower permeability and porosity. Another interesting fact that is noticed in this case is that, unlike the horizontal fracture case, the lateral heating enhances vertical separation in the cavity, as both the Soret effect and buoyancy-driven convection combine their effect and create separation in the cavity.

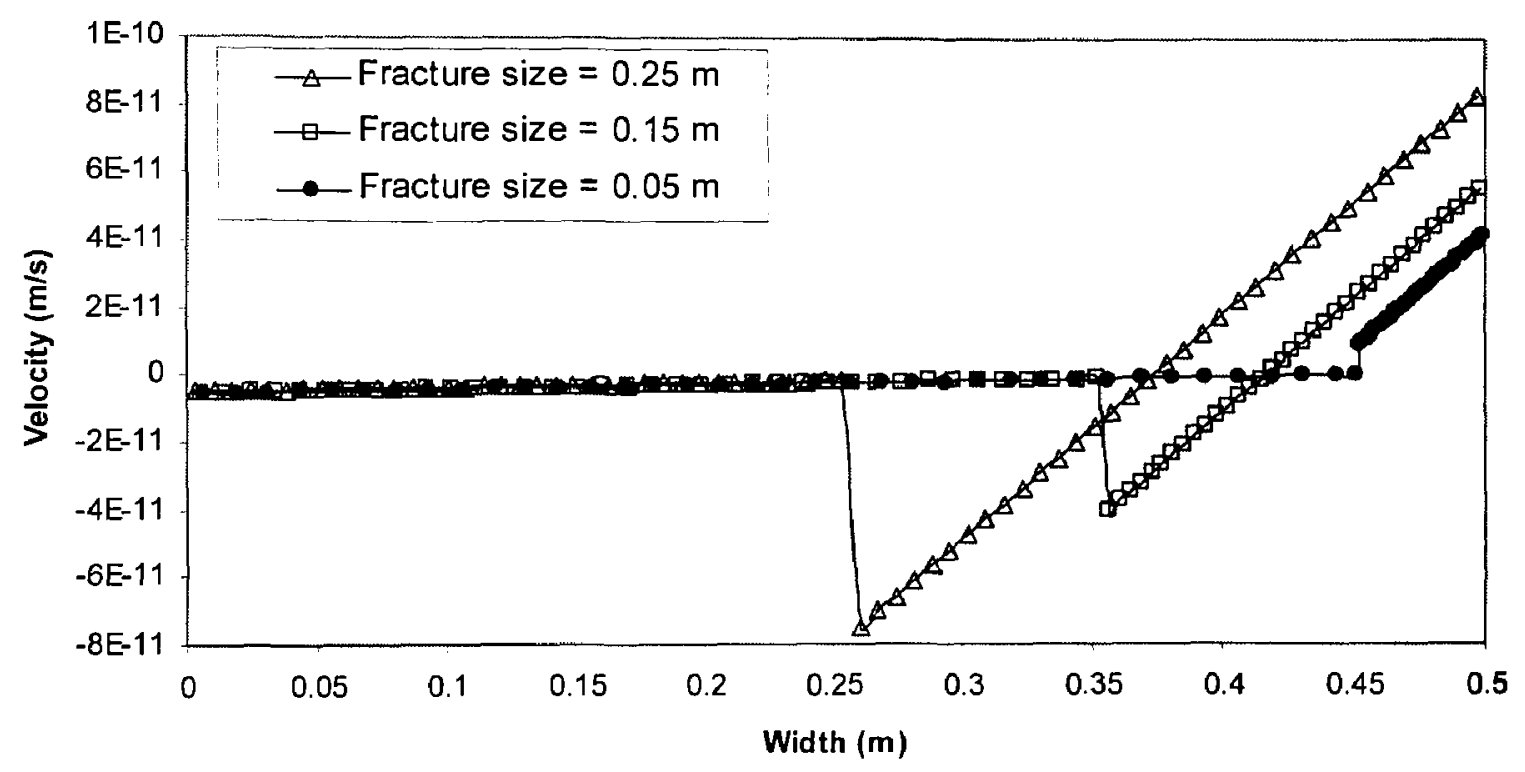

Figure 32: Vertical fracture: Velocity horizontally inside the cavity

As the fracture's width decreases to $0.15 \mathrm{~m}$, once again a counter-clock wise rotating convective cell appears in the fracture, and more fluid is penetrating the primary domain compared to the previous case. This is due to the fact that the flow is moving at a faster rate in the fracture, and hence can force the fluid out into the primary domain further. The velocity along the width of the cavity at quarter of the height is examined, and it confirms that the velocity of the fluid in the fracture is moving faster than in the previous case (fracture with of $0.25 \mathrm{~m}$ ). 
As the fracture's width decreases further to $0.05 \mathrm{~m}$, it is noticed that the counter-clock wise rotating convective cell disappears. Instead, a half cell appears in the fracture, with all the flow going up along the hot wall. Furthermore, almost all of the fluid penetrates the primary domain, and in fact a semi convective cell appears in the primary domain, with the eye of the cell close to the interface of the fracture and primary domain. This fact can be explained by considering the fact that permeability and porosity in the fracture are higher compared to the primary domain; therefore, buoyancy-driven convection is stronger in the fracture, which causes the flow to go up the hot wall. However, the fracture is really small in width, and a less permeable and less porous domain is also present, where thermal diffusion is dominant. Therefore, the flow is moving slowly in the primary domain; when it reaches the fracture, it flow speeds up significantly. This is further evident in the velocity profile, which shows a uniform velocity throughout the primary domain then a jump and increase in the velocity as fracture is reached.

\subsubsection{Comparison of concentration inside the cavity}

To analyze the effect of Soret and buoyancy-driven convection in the cavity and the influence of fracture on it, the fluid composition variation is examined. In Figure 33, it is evident that when the fracture size is $2.5 \mathrm{~m}$, there exists a clear distinction between then the fractured and primary domain. In the primary domain, the concentration contours are almost straight and vertical, while in the fracture domain, the concentration contours are tilted at an angle. The straight concentration contours in the primary domain represent the separation caused by the Soret effect, while the tilted concentration contours in the fractured domain represents the presence of buoyancy-driven convection. This finding confirms the earlier observation made when examining the flow behaviour in the cavity. As the fracture size is decreased to $1.5 \mathrm{~m}$, tilts in the concentration contours are less apparent; nevertheless, it is still present. The Soret effect causes vertical separation in the primary domain, and the heavier component, THN, continues to accumulate near the cold side of the cavity. As the fracture size is decreased to $0.5 \mathrm{~m}$, almost the entire cavity is dominated by the Soret effect, and concentration contours are almost entirely vertical. 
However, there exists a slight tilt near the top wall of the cavity due to the buoyancydriven convection, which is caused by the presence of fracture.

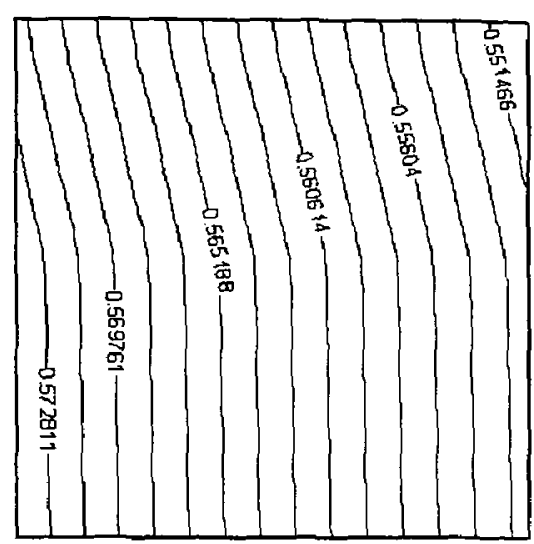

a). Fracture size $=2.5 \mathrm{~m}$

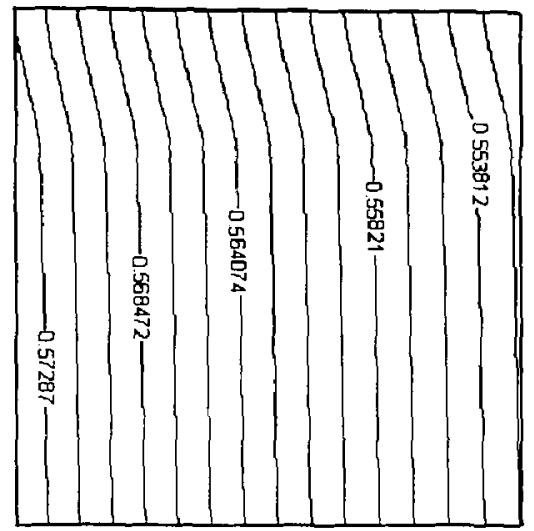

b). Fracture size $=1.5 \mathrm{~m}$

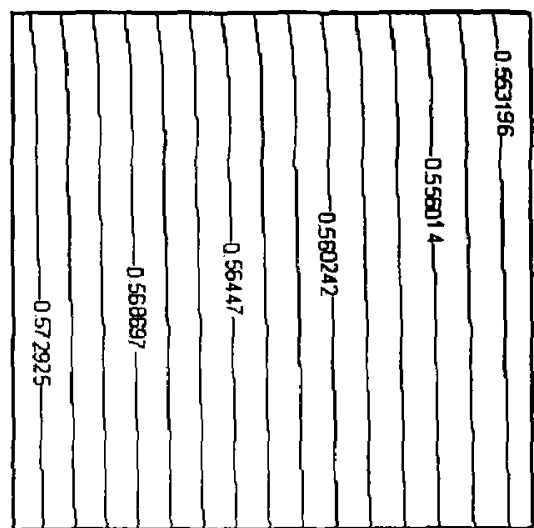

c). Fracture size $=0.5 \mathrm{~m}$

Figure 33: Horizontal fracture case: Concentration contours

The fluid composition variations are shown in Figure 34 for the vertical fracture case. When the fracture size is $0.25 \mathrm{~m}$, the concentration contours are tilted and the heavier component, THN, is accumulating near the cold wall of the cavity. The tilt in the concentration contours indicates that both the Soret effect and buoyancy-driven convection are contributing to the separation in the cavity. As the fracture size is reduced to $0.15 \mathrm{~m}$, the concentration contours become less tilted. This is due to the fact that the Soret effect is becoming dominant in the cavity as the permeable fracture becomes smaller. Finally, when the fracture size is reduced to $0.05 \mathrm{~m}$, the concentration contours become vertical, indicating the Soret effect is dominant in the cavity. 


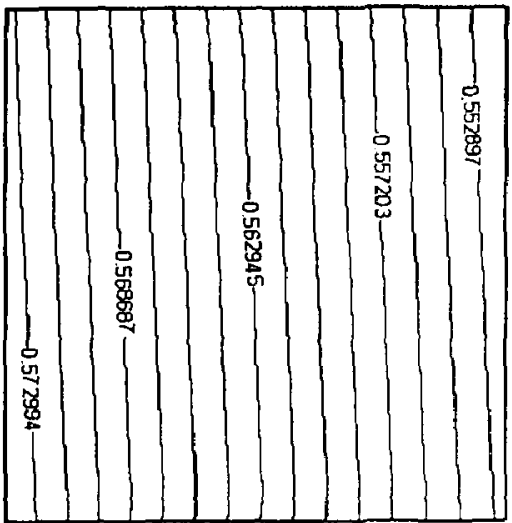

a). Fracture size $=0.25 \mathrm{~m}$

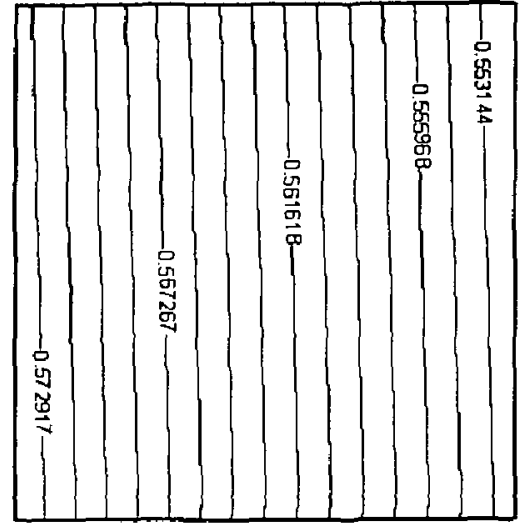

b). Fracture size $=0.15 \mathrm{~m}$

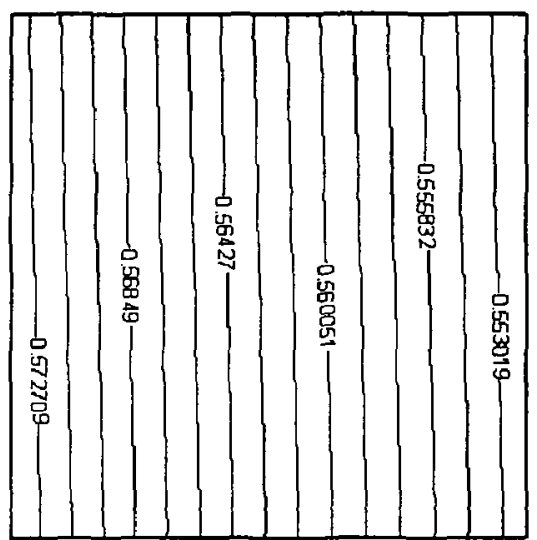

c). Fracture size $=0.05 \mathrm{~m}$

Figure 34: Vertical fracture case: Concentration contours

\subsubsection{SORET COEFFICIENT COMPARISON FOR HORIZONTAL AND VERTICAL FRACTURE CASES}

In heterogeneous porous media, the Soret coefficient, $\mathrm{S}_{\mathrm{T}}$, will be influenced by regiondependent convection flow. In order to investigate this effect, the Soret coefficient distribution along the central vertical line for the horizontal fracture case and the Soret coefficient distribution along the central horizontal line for the vertical fracture case, in the porous cavity, were examined.

For the horizontal fracture case, it is noticed that the Soret coefficient in the primary domain varies linearly indicating the dominance of the Soret effect in the lower part of the cavity, in other words, the primary domain, as shown in Figure 35. However, as the fracture is reached, the Soret coefficient slope changes and the variation becomes more dramatic; the variation also has a slight curve in it. This indicates the presence and dominance of the buoyancy-driven convection in the upper part of the cavity, or where the fracture is present. The same pattern is observed for all three sizes of fracture. This pattern also confirms the trends and observation made earlier. 


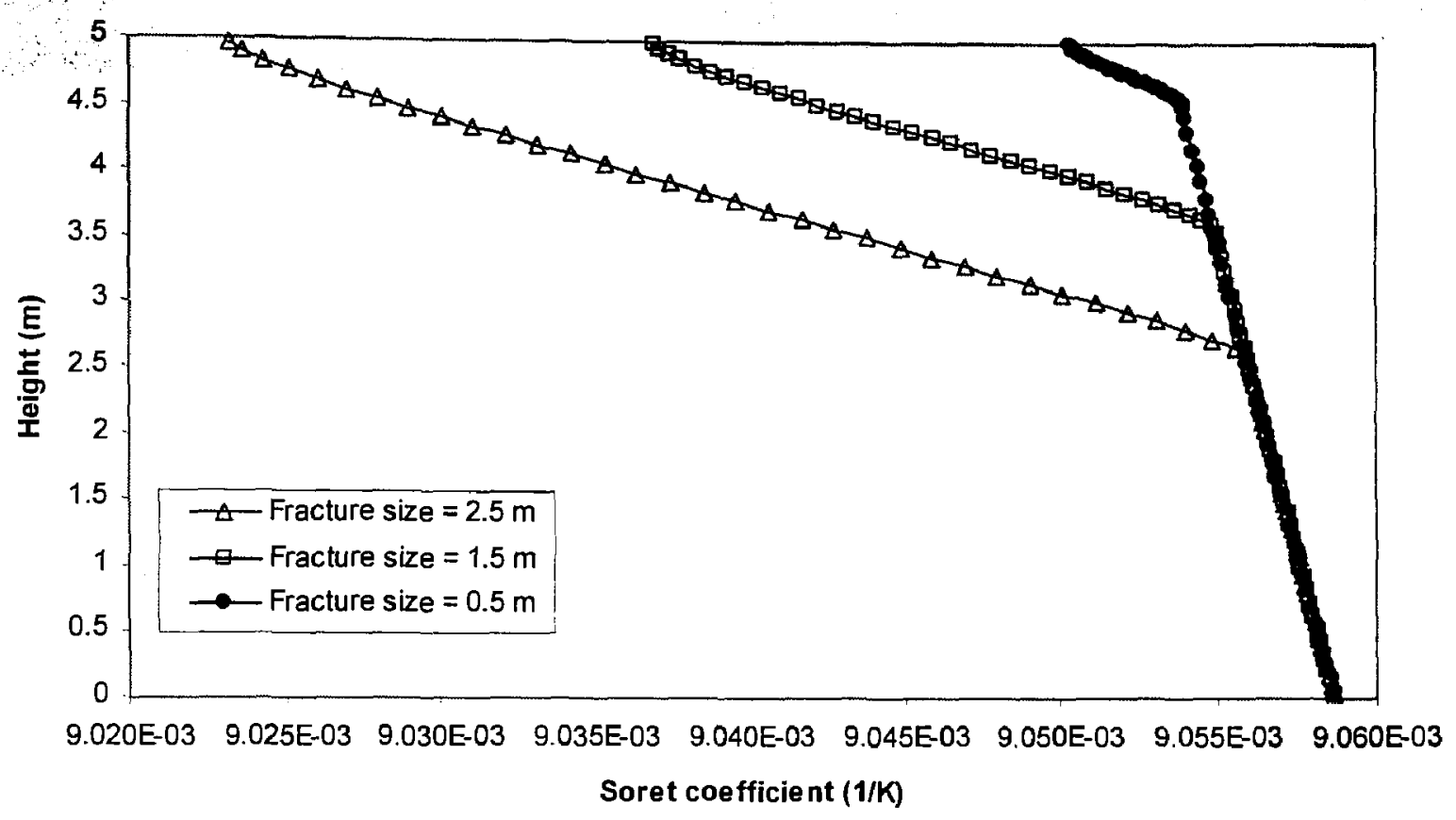

Figure 35: Soret coefficient for horizontal fracture case

For the vertical fracture case, a linear variation is observed for all three sizes of the fracture, with very slight variation in the fractured domain, as seen in Figure 36. There are two reasons for this pattern. First, the Soret effect is dominant in the primary domain due to lateral heating, and even though the fracture is present and buoyancy-driven convection exists in the fracture, the convection contributes to the separation in the cavity, therefore assisting the Soret coefficient instead of altering it. Secondly, the size of the fracture is relatively small, and therefore, unlike the horizontal fracture where the sizes of the fractures are relatively large, the buoyancy-driven convection becomes further ineffective in dominating the entire cavity. 


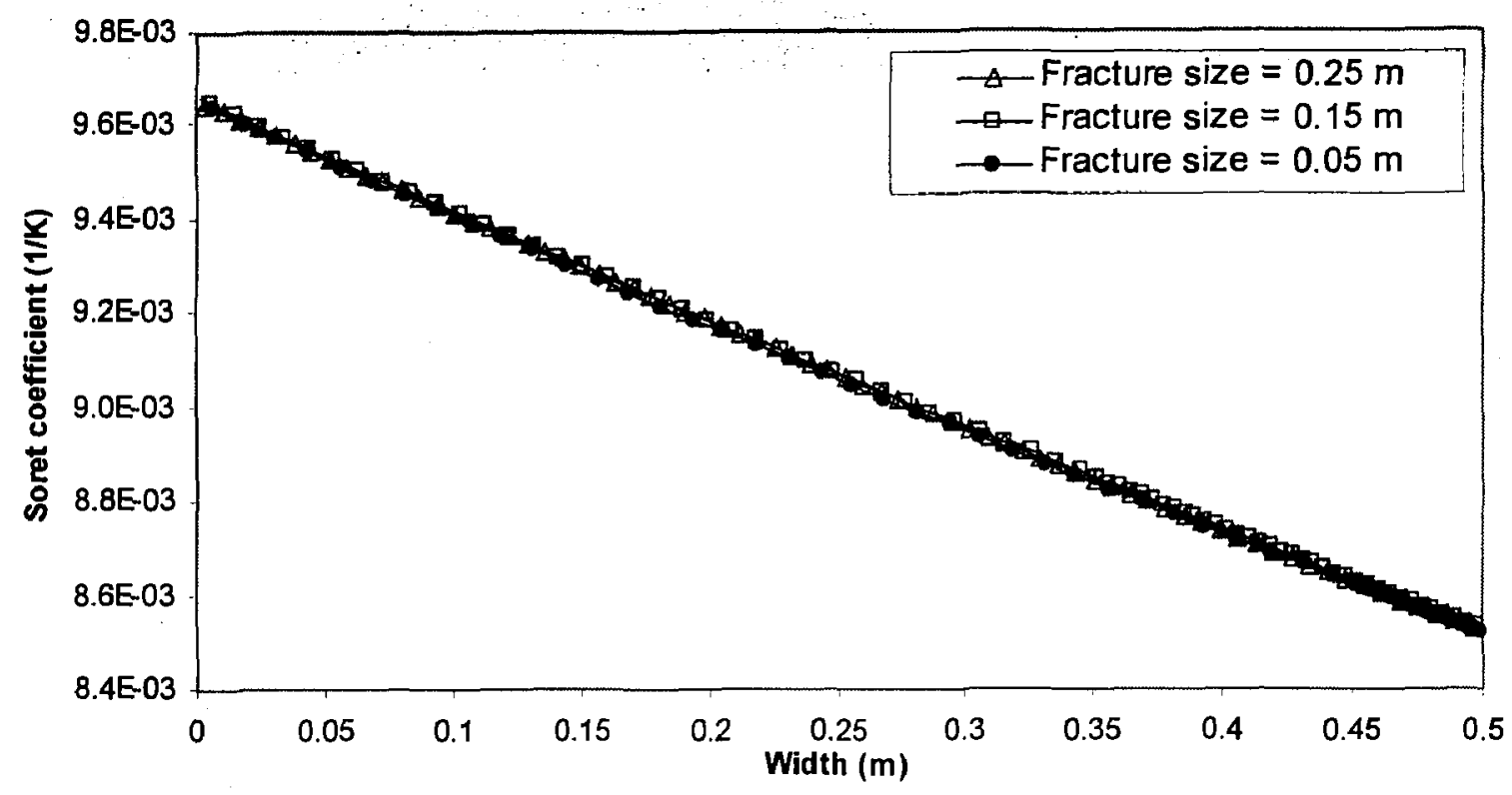

Figure 36: Soret coefficient for the vertical fracture case

\subsection{SUMMARY}

This chapter investigates the interaction between thermal diffusion and buoyancy driven convection and the influence of fractures on a single-phase hydrocarbon binary mixture system. The numerical simulation utilized a multi-porosity/multi-permeability model to simulate both horizontal and vertical fractures in a porous media. The sizes of the fractures were varied to make the investigation more realistic. In the analysis, the flow characteristics, the velocity in the cavity, the component concentration gradients, along with the Soret coefficient, has been used to investigate the behaviour of thermal diffusion and buoyancy-driven convection, and the effect of fracture. The analysis reveals that when a horizontal fracture is present in the porous media, a strong convective cell appears in the porous and permeable fracture due to the dominance of buoyancy-driven convection. It was also noticed that the fluid starts to leaks into the primary domain near the cold wall and re-enters the fracture after the center of the cavity is reached. This leakage and re-entry creates a central plume in the cavity, which gets narrower as the fracture size is decreased. Furthermore, it was found that even though buoyancy-driven convection was dominant in the fracture, the Soret effect was dominant in the primary 
domain. The heavier component, THN, migrated to the cold wall due to the Soret effect. The Soret coefficient variation vertically in the cavity confirmed these observations. For the case of horizontal fracture, once again buoyancy-driven convection is observed in the fractured cavity. The fast moving flow in the fracture pushes the fluid out of the fracture and into the primary domain near the top of the cavity, and it causes the fluid to re-enter the fracture near the bottom of the cavity. As the fracture size decreases, the flow gets stronger and more fluid penetrates the primary domain. When the fracture size is $0.05 \mathrm{~m}$, a half convective cell appears in the fracture and a semi-convective cell appears in the primary domain. The half convective cell pushes the fluid up along the hot wall, and the semi-convective cell circulates the fluid in the primary domain. It was noticed that, unlike the horizontal fracture, in the vertical fracture case, the Soret effect and buoyancy-driven convection both combine their effect and enhance the separation in the cavity. However, as the fracture size decreases, the Soret effect dominates the entire cavity. 


\section{CHAPTER 6: CONCLUSION}

The process of thermal diffusion, also known as the Soret effect, was named after Ludwig and Soret to commemorate their momentous discovery. The process of thermal diffusion can be described as the separation of components when a temperature gradient is applied to a previously homogeneous mixture. Since the discovery of this process, many researchers and scientists have studied this process both experimentally and theoretically. Today, researchers continue to investigate and comprehend this phenomenon in greater details and contribute to understanding of this essential mechanism in natural processes.

The mathematical model that describes the thermal diffusion process can be fully established based on the conservation laws of transport theory and laws of nonequilibrium thermodynamics. Furthermore, by utilizing the reasonable and realistic Curie's assumptions, and by including the concept of net heat of transport, the theory is expanded to include multicomponent mixtures under non-ideal conditions. This mathematical model is known as the Firoozabadi model.

Unlike other theoretical models, such as Haase or Kempers, the Firoozabadi model utilizes the activation energy and the energy of viscous flow, which are both related to the dynamic viscosity of the fluid. Furthermore, in this model the viscosity is a parameter, which is considered not part of the thermodynamics, therefore highlighting and emphasising the Soret effect. Even though, the Firoozabadi model gives the formulation for the estimation of thermal and molecular diffusion coefficients, the accuracy of the calculation depends on all of the thermodynamic parameters, such as density, enthalpy, internal energy and fugacity. Therefore, a good equation of state, such as Peng-Robinson, is crucial for the estimation.

The objective of this study is to verify the application of the Firoozabadi model by first comparing the thermal and molecular diffusion coefficients of three binary mixtures with benchmark experimental values. The three components that constitutes the mixtures includes, n-Dodecane, Isobutylbenzene, and 1, 2, 3, 4-Tetrahydronaphthalene, and are 
representative of the crude oil chemical families. The binary mixtures have a fixed concentration of $50 \mathrm{wt} \%$ and are maintained at a mean temperature of $25^{\circ} \mathrm{C}$ and under atmospheric pressure. The analysis reveals an excellent agreement between the experimental and numerical values. The analysis was taken one step further and a ternary mixture was utilized to calculate the thermal diffusion coefficients. The ternary mixture had a fixed concentration of $33 \mathrm{wt} \%$ and was again under atmospheric pressure and 25 ${ }^{\circ} \mathrm{C}$. To the best of the authors knowledge, this study is the first to successfully report the comparison between experimental and numerical results for a ternary hydrocarbon mixture. The accuracy of the numerical results can be further improved by utilizing more accurate thermodynamic properties, such as the molar volume at boiling point and the viscosity of the pure components. The study also successfully correlated the thermal diffusion coefficients of three binary mixtures to the thermal diffusion coefficients of the ternary mixture.

Convection also has a great influence on the thermal diffusion process in hydrocarbons contained in a porous media. Therefore, a two-dimensional numerical model was used to investigate the thermo-solutal convection in a porous media. A forward difference numerical scheme was solved using an iterative solution strategy known as the SIMPLE algorithm. Once again, the three above-mentioned binary mixtures were used, and a range of permeability values was used to make the analysis more realistic. The flow analysis revealed a consistent behaviour between all three binary mixtures. In all three cases, the heavier component migrated to the cold wall, which is consistent with the experimental observations. The THN-Dodecane mixture was influenced the most by the Soret effect and buoyancy-driven convection and, therefore, had the greatest separation in the cavity. Furthermore, three regimes were discovered based on the permeabilities. The first regime, where the permeability values are low, the Soret effect dominates the entire cavity. The second regime, where the permeability values are mid-range, the Soret effect and the buoyancy-driven convection combine their effect to enhance the separation in the cavity. Finally, in the third regime, where the permeability values are high, the buoyancydriven convection dominates the cavity, and the Soret effect diminishes due to mixing. 
Finally, the effect of fractures in the porous media on the thermo-solutal convection was investigated. Once again, a two-dimensional numerical model was used in the numerical simulation. The simulation analyzed both horizontal and vertical fractures in the porous media using a multi-porosity/multi-permeability model. In order to make the analysis more realistic, the size of the fractures was varied. The analysis revealed that when a horizontal fracture is present in the cavity, the fluid leaks from the fracture and into the primary domain, creating a central plume in the cavity. The plume becomes narrower as the fracture size is reduced because the fluid has to travel further distance, hence elongating the plume. The flow also moved faster in the fracture compared to the primary domain. This was due to the fact that buoyancy-driven convection is dominant in the fracture, while the Soret effect is dominant in the primary domain. As for the case of vertical fracture, it was noticed that the flow penetrated the primary domain near the top of the cavity and re-entered near the bottom of the cavity. As the size of the fracture decreased, more fluid penetrated the primary domain. When the fracture size was reduced to $0.05 \mathrm{~m}$, a half convective cell appeared and pushed the fluid up along the hot wall of the cavity, while a semi-convective cell appeared in the primary domain that circulated the flow in the cavity. It was also revealed that when the vertical fracture size was half the width of the cavity, the Soret effect and buoyancy-driven convection combine their effect and enhance the separation in the cavity. However, as the fracture size decreases, the Soret effect influences the entire cavity.

As for future research, the two-dimensional model can be extended to a threedimensional one. Furthermore, a ternary mixture can be utilized in the investigation of thermo-solutal convection. 


\section{REFERENCES}

[1] Nield D.A. and Bejan A., (1999), Convection in porous media, Springer-Verlag, New York

[2] Ingham, D.B. and Pop I. (1998). Transport phenomena in porous media, Pergamon, Oxford

[3] Ingham, D.B. and Pop I. (2002). Transport phenomena in porus media II, Pergamon, New York

[4] Schmidt R.J. and Milverton S.W., (1935). On the Instability of the Fluid When Heated from Blow, Proc. R. Soc., (A152), London

[5] Kohler W. and Muller B., (1995). Soret and Mass Diffusion Coefficients of Toluene/n-Hexane Mixtures, J. Chem. Phys., 103:10, 4367-4370

[6] Kohler W. and Wiegand S. (2002). Thermal Nonequilibrium Phenomena in Fluid Mixtures, Springer, Berlin

[7] Costeseque P., Pollak, T., Platten J., Marcoux M., (2004). Simultaneous Evaluation of Soret and Fick Coefficients in a Free and a Packed Vertical Gradient Soret Cell, $6^{\text {th }}$ International Meeting on Thermodiffusion, Varenna, Italy

[8] Platten J.K., Bou-Ali M. M. , Costeseque P. , Dutrieux J. F. , Kohler W. , Leppla C. , Wiegand S. , Wittko G. , (2003). Benchmark Values for Soret, Thermal Diffusion and Diffusion Coefficients of Three Binary Organic Liquid Mixtures, Philosophical Magazine, 83, 1965 - 1971 
[9] Platten J.K., Bou-Ali M. M., Leahy-Dios, A., Firoozabadi, A., (2005). Measurements of molecular and thermal diffusion coefficients in ternary mixture, Journal of Chemical Physics, 122, 1-12

[10] Shukla K., Firoozabadi A., (1998). A New Model of Thermal Diffusion Coefficients in Binary Hydrocarbon Mixtures, Ind. Engrg. Chem. Res., 37, 3331-3342

[11] Firoozabadi A., Ghorayeb K., Shukla K., (2000). Theoretical Model of Thermal Diffusion Factors in Multicomponent Mixtures, Al Ch E J., 46, 892-900

[12] Lorenz M. and Emery Jr. A. H., (1959). The Packed Thermal Diffusion Column, Chemical Engineering Science, 11:1, 16-23

[13] Jamet Ph., Costeseque P., and Fargue D., (1996). Determination of the Effective Transport Coefficients for the Separation of Binary Mixtures of Organic Compounds into Packed Thermal Diffusion Columns, Chemical Engineering Science, 51: 19, 4463-4475

[14] Benano-Melly L. B., Caltagirone J. P., Faissat B., Montel F., Costeseque P., (2001). Modeling Soret Coefficient Measurement Experiments in Porous Media Considering Thermal and Solutal Convection, International Journal of Heat and Mass Transfer, 44:7, 1285-1297

[15] Riley M. and Firoozabadi A., (1998). Compositional Variation in Hydrocarbon Reservoirs with Natural Convection and Diffusion, AlChE J. , 44, 452-464

[16] Chacha M. and Saghir M.Z., (2003). Influence of Thermal Boundary Conditions on the Double-Diffusive Process in a Binary Mixture, Philosophical Magazine, 83:17-18, 2109-2129

[17] Gershumi G.Z., Zhukhovitsky E.M., (1981). Convective Instability of a Fluid in a Vibration Field under Conditions of Weightless, Fluid Dynamics, 16:4, 498-504 
[18] Savino R., Monti R., Piccirillo M., (1998). Thermovibrational Convection in a Fluid Cell, Computers and Fluids, 27, 923-939

[19] Chacha M., Faruque D., Saghir M.Z., Legros J.C., (2002). Solutal Thermodiffusion in Binary Mixture in the Presence of g-jitter, International Journal of Thermal Sciences, $41: 9,899-911$

[20] Sahimi, M., (1995). Flow and Transport in Porous media and fractured rock: from classical methods to modern approaches, $\mathrm{VCH}$, Weinheim

[21] Alboin C., Jaffre, J., Roberts, J.E., Wang, X., and Serres, C., (1999). Domain decomposition for some transmission problems in flow in porous media, Lecture Notes in Physics, 552, 22-34

[22] Jiang C.G., Saghir M.Z. and Kawaji M., (2004). Thermo-Solutal Convection in Heterogeneous Porous Media, International Conference on Porous Media and Applications, Portugal

[23] Haase R., (1969). Thermodynamics of Irreversible Processes, Chapter 4, Addison Wesley, London

[24] Rutherford, W.M. and Roof, J.G., (1959). Thermal Diffusion in Methane n-Butane Mixture in the Critical Region, J. Phys. Chem., 63, 1506-1511

[25] Dougherty, E. L., Drickamer, H. G., (1955). Thermal Diffusion and Molecular Motion in Liquids, Journal of Physical Chemistry, 59, 443-449

[26] Mortimer R.G. and Eyring H., (1980). Elementary Transition-State Theory of the Soret and Dufour Effects, Proceedings of the National Academy of Sciences of the United States of America - Physical Sciences, 77:4, 1728-1731 
[27] De Haro M.L., Cohen E.G.D., Kincaid J.M., (1983). The Enskog Theory for Multicomponent Mixtures: 1. Linear Transport-Theory, J. Chemical Physics, 78:5, 27462759

[28] Kincaid J.M., De Haro M.L., Cohen E.G.D., (1983). The Enskog Theory for Multicomponent Mixtures: 2. Mutual Difusion, J. Chemical Physics, 79:9, 4509-4521

[29] Guy A.G. (1986), Prediction of Thermal-Difusion in Binary -Mixtures of Nonelectrolyte Liquids by the Use of Nonequilibrium Thermodynamics, Int. J. Thermodynamics, 7:3, 563-572

[30] Bearman R.J., Kirkwood J.G., Fixman M., (1958). Statistical-Mechanical Theory of Transport Processes: The Heat of Transport in Binary Liquid Solutions, Advances in Chemical Physics, 1, 1-13

[31] Kempers L.J.T.M., (1989). A Thermodynamic Theory of the Soret Effect in a Multicomponent Liquid, J. Chem. Phys., 90:11, 6541-6548

[32] Onsager, L., (1931). Reciprocal relations in Irreversible processes: I, Phys. Rev., 37, 405

[33] Patankar, S.V. and Spalding, D.B. (1972). A Calculation Procedure for Heat, Mass and Momentum Transfer in Three-dimensional Parabolic Flows, Int. J. Heat Mass Transfer, 15:10, 1787-1806

[34] Patankar S.V., (1980). Numerical Heat Transfer and Fluid Flow, Hemisphere Publishing Corporation, Taylor and Francis Group, New York

[35] Peyret R. and Taylor T. D., (1983). Computational Methods in Fluid Flows, Springer, New York 
[36] Hirsch C. (1990). Numerical Computation in Internal and External Flows, Wiley, New York

[37] Jiang C.G., Saghir M.Z., Kawaji M. and Ghorayeb K., (2004). Two-Dimensional Numerical Simulation of Thermo-Gravitational Convection in a Vertical Porous Column Filled with a Binary Fluid Mixture, International Journal of Thermal Sciences (in press)

[38] Saghir M.Z., Jiang C.G., Derawi S.O., Kawaji M., (2004). Comparison between Theoretical and Experimental Data for the Soret Coefficient for Water-Alcohol Mixtures, $6^{\text {th }}$ International Meeting on Thermodiffusion, Varenna, Italy

[39] Faruque, D., Chacha, M., Saghir, M.Z., and Ghorayeb, K., (2004). Compositional Variation Considering Diffusion and Convection for Binary Mixture in Porous Media, Journal of Porous Media, 7:2, 1-19

[40] Platten, J. K., (2002). Proceedings of the $5^{\text {th }}$ International Meeting of Thermodiffusion (IMT5), Philos. Mag., 83, 17-18

[41] Dutrieux, J. F., Platten, J. K., Chavepeyer, G., Bou-Ali, M. M., (2002). On the measurement of positive Soret coefficients, J. Phys. Chem., 106, 6104-6110

[42] Barrenblatt, G. D., (1960). Basic concepts in the theory of homogenous liquids in fissured rocks; Journal of Applied Math, 24, 1286-1303

[43] Warren, J. E., Root, P. J., (1963). The behaviour of naturally fractured reservoirs, SPEJ, 245-255

[44] Kazemi, H., Merrill, L. S., Porterfild, K. L., and Zeman, P. R., (1976) "Numerical Simulation of water-oil flow in naturally fractured reservoirs, SPEJ, 317-326 
[45] Storesletten, L., (2003). Anistropy effects on convective flow in porous media: Part I and part II, current issue on heat and mass transfer in porous media, Procs. of the NATO Advanced Study Institute on Porous Media, Neptun-Olimp, Black Sea, Romania, 529546

[46] Saghir, M. Z., Islam, M. R., (1999). Double diffusive convection dual-permeability, dual-porosity porous media, International Journal of Heat and Mass Transfer, 42, 437454 


\section{APPENDIX A}




\section{APPENDIX A}

$\mathrm{A}=\mathrm{THN}=$ Tetrahydronaphtalene (Tetraline) C.A.S. 119-64-2 $\left(\mathrm{C}_{10} \mathrm{H}_{12}\right)$

$\mathrm{B}=$ Dodecane $=\mathrm{n}$-Dodecane $\left(\mathrm{C}_{12} \mathrm{H}_{26}\right)$

$\mathrm{C}=\mathrm{IBB}=$ Isobutylbenzene $\left(\mathrm{C}_{10} \mathrm{H}_{14}\right)$ C.A.S. 538-93-2

Initial Mass fraction of each component

$c_{A}=0.34 w t \%$
$c_{B}=0.33 w t \%$
$c_{c}=0.33 w t \%$

Molecular Weight

$\mathrm{M}_{\mathrm{A}}=132.21 \mathrm{~g} / \mathrm{mol}$

$\mathrm{M}_{\mathrm{B}}=170.34 \mathrm{~g} / \mathrm{mol}$

$\mathrm{M}_{\mathrm{C}}=134.22 \mathrm{~g} / \mathrm{mol}$

Number of Moles

$\frac{c_{i}}{M_{i}}$

$\mathrm{C}_{10} \mathrm{H}_{12}=\mathrm{THN}=\frac{0.34}{132.21 \mathrm{~g} / \mathrm{mol}}=0.00257166 \mathrm{~mol}$

$\mathrm{C}_{12} \mathrm{H}_{26}=$ Dodecane $=\frac{0.33}{170.34 \mathrm{~g} / \mathrm{mol}}=0.00193730 \mathrm{~mol}$

$\mathrm{C}_{10} \mathrm{H}_{14}=\mathrm{IBB}=\frac{0.33}{134.22 \mathrm{~g} / \mathrm{mol}}=0.00245861 \mathrm{~mol}$

Total $=\sum_{i=1}^{n=3} \frac{c_{i}}{M_{i}}=0.00257166+0.00193730+0.00245861=0.00696757 \mathrm{~mol}$ 
Mole fraction of each component

$$
\begin{aligned}
& x_{i}=\frac{c_{i} / M_{i}}{\text { Total }} \\
& x_{A}=\frac{0.00257166}{0.00696757}=0.3691 \\
& x_{B}=\frac{0.00193730}{0.00696757}=0.2780 \\
& x_{C}=\frac{0.00245861}{0.00696757}=0.3529
\end{aligned}
$$

Total Molecular weight

$$
\begin{aligned}
& \mathrm{M}=\mathrm{x}_{\mathrm{A}} \mathrm{M}_{\mathrm{A}}+\mathrm{x}_{\mathrm{B}} \mathrm{M}_{\mathrm{B}}+\mathrm{x}_{\mathrm{c}} \mathrm{M}_{\mathrm{c}}=143.5201748 \mathrm{~g} / \mathrm{mol} \\
& \therefore \mathrm{M}=0.14352 \mathrm{~kg} / \mathrm{mol}
\end{aligned}
$$

Density

$$
\begin{array}{ll}
\rho_{\mathrm{A}}=965.47 \mathrm{~kg} / \mathrm{m}^{3} & \text { At } 25^{\circ} \mathrm{C} \\
\rho_{\mathrm{B}}=784.800 \mathrm{~kg} / \mathrm{m}^{3} & \text { At } 20^{\circ} \mathrm{C} \\
\rho_{\mathrm{C}}=849.079 \mathrm{~kg} / \mathrm{m}^{3} & \text { At } 20^{\circ} \mathrm{C}
\end{array}
$$


Pressure

$101300.00 \mathrm{~Pa}$

Temperature

$298 \mathrm{~K}$

Physical Properties

\begin{tabular}{lllll}
\hline & Asentric factor $\omega$ & Critical T $(\mathrm{K})$ & Critical P $(\mathrm{Pa})$ & Molecular weight $[\mathrm{g} / \mathrm{mol}]$ \\
\hline THN & 0.3030 & 719.00 & $0.3515 \times 10^{7}$ & 132.21 \\
Dodecane & 0.5764 & 658.20 & $0.1820 \times 10^{7}$ & 170.34 \\
IBB & 0.3780 & 650.00 & $0.3141 \times 10^{7}$ & 134.22 \\
\hline
\end{tabular}

Critical Volume $\left[\mathrm{m}^{3} / \mathrm{kg}\right]$

\begin{tabular}{ll}
\hline THN & $3.335 \times 10^{-3}$ \\
Dodecane & $4.203 \times 10^{-3}$ \\
IBB & $3.577 \times 10^{-3}$ \\
\hline
\end{tabular}

Viscosity [mPascal Seconds]

THN $2020.0 \times 10^{-3}$

Dodecane $1374.5 \times 10^{-3}$

IBB $\quad 1850.0 \times 10^{-3}$

Molar volume at boiling point $\left[\mathrm{cm}^{3} / \mathrm{mol}\right]$

\begin{tabular}{ll}
\hline THN & $1.367 \times 10^{2}$ \\
Dodecane & $2.286 \times 10^{2}$ \\
IBB & $1.864 \times 10^{2}$ \\
\hline
\end{tabular}




\begin{tabular}{ll}
\hline & Tau \\
\hline THN & 4.0 \\
Dodecane & 4.0 \\
IBB & 4.0 \\
\hline
\end{tabular}

\begin{tabular}{ll}
\hline & Binary Interactive Coefficients \\
\hline THN & 0.00 \\
Dodecane & 0.00 \\
IBB & 0.00 \\
\hline
\end{tabular}

It is quite common that investigators use different systems of units in their experiments and their theories, such is the case with Platten's experimental data [9] and Firoozabadi's theoretical model $[10,11]$. In order to obtain accurate results from the comparison of experimental data with values obtained by using a theoretical model, correct conversion of units is imperative. A detailed derivation and conversion is presented below for a ternary mixture. 
Relationship between the mole fraction gradient and the mass fraction gradient of component 1

$$
\begin{aligned}
& x_{1}=\frac{c_{1} / M_{1}}{c_{1} / M_{1}+c_{2} / M_{2}+1 / M_{3}-c_{1} / M_{3}-c_{2} / M_{3}} \\
& \frac{\partial x_{1}}{\partial X}=\frac{\frac{d c_{1}}{d x} \frac{1}{M_{1}}}{\left(c_{1} / M_{1}+c_{2} / M_{2}+1 / M_{3}-c_{1} / M_{3}-c_{2} / M_{3}\right)} \\
& \frac{\frac{c_{1}}{M_{1}}}{\left(c_{1} / M_{1}+c_{2} / M_{2}+1 / M_{3}-c_{1} / M_{3}-c_{2} / M_{3}\right)^{2}} \frac{d c_{1}}{d x} \frac{1}{M_{1}}- \\
& \frac{\frac{c_{1}}{M_{1}}}{\left(c_{1} / M_{1}+c_{2} / M_{2}+1 / M_{3}-c_{1} / M_{3}-c_{2} / M_{3}\right)^{2}} \frac{d c_{2}}{d x} \frac{1}{M_{2}}+ \\
& \frac{\frac{c_{1}}{M_{1}}}{\left(c_{1} / M_{1}+c_{2} / M_{2}+1 / M_{3}-c_{1} / M_{3}-c_{2} / M_{3}\right)^{2}} \frac{d c_{1}}{d x} \frac{1}{M_{3}}+ \\
& \frac{\frac{c_{1}}{M_{1}}}{\left(c_{1} / M_{1}+c_{2} / M_{2}+1 / M_{3}-c_{1} / M_{3}-c_{2} / M_{3}\right)^{2}} \frac{d c_{2}}{d x} \frac{1}{M_{3}} \\
& \frac{\partial x_{1}}{\partial X}=\frac{\partial M_{2}+c_{2} / M_{2} M_{1}+1 / M_{3} M_{1}-c_{1} / M_{2}-c_{2} / M_{3} M_{1}-M_{X}+c_{3} M_{L}}{\left(c / c_{1}+c_{2} / M_{2}+1 / M_{3}-c_{1} / M_{3}-c_{2} / M_{3}\right)^{2}}+ \\
& \frac{-c_{1} / M_{1} M_{2}+c_{1} / M_{1} M_{3}}{\left(c_{1} / M_{1}+c_{2} / M_{2}+1 / M_{3}-c_{1} / M_{3}-c_{2} / M_{3}\right)^{2}} \frac{d c_{2}}{d x}
\end{aligned}
$$




$$
\begin{aligned}
& \frac{\partial x_{1}}{\partial X}=\frac{c_{2} / M_{2} M_{1}+1 / M_{3} M_{1}-c_{2} / M_{3} M_{1}}{\left(c_{1} / M_{1}+c_{2} / M_{2}+1 / M_{3}-c_{1} / M_{3}-c_{2} / M_{3}\right)^{2}} \frac{d c_{1}}{d x}+ \\
& \frac{-c_{1} / M_{1} M_{2}+c_{1} / M_{1} M_{3}}{\left(c_{1} / M_{1}+c_{2} / M_{2}+1 / M_{3}-c_{1} / M_{3}-c_{2} / M_{3}\right)^{2}} \frac{d_{c_{2}}}{d x}
\end{aligned}
$$

$$
\begin{aligned}
& \frac{\partial x_{1}}{\partial X}=\frac{\frac{M_{2}+c_{2} M_{3}-c_{2} M_{2}}{M_{1} M_{2} M_{3}}}{\left(c_{1} / M_{1}+c_{2} / M_{2}+1 / M_{3}-c_{1} / M_{3}-c_{2} / M_{3}\right)^{2}} \frac{d c_{1}}{d x}+ \\
& \frac{\frac{c_{1} M_{2}-c_{1} M_{3}}{M_{1} M_{2} M_{3}}}{\left(c_{1} / M_{1}+c_{2} / M_{2}+1 / M_{3}-c_{1} / M_{3}-\frac{\left.c_{2} / M_{3}\right)^{2}}{d x}\right.} \frac{d c_{2}}{d x} \\
& \nabla x_{1}=\frac{M^{2}}{M_{1} M_{2} M_{3}}\left(M_{2}+c_{2} M_{3}-c_{2} M_{2}\right) \nabla c_{1}+\frac{M^{2}}{M_{1} M_{2} M_{3}}\left(c_{1} M_{2}-c_{1} M_{3}\right) \nabla c_{2}
\end{aligned}
$$

110 
Relationship between the mole fraction gradient and the mass fraction gradient of component 2

$$
\begin{aligned}
& x_{2}=\frac{c_{2} / M_{2}}{c_{1} / M_{1}+c_{2} / M_{2}+1 / M_{3}-c_{1} / M_{3}-c_{2} / M_{3}} \\
& \frac{\partial x_{2}}{\partial X}=\frac{\frac{d c_{2}}{d x} \frac{1}{M_{2}}}{\left(c_{1} / M_{1}+c_{2} / M_{2}+1 / M_{3}-c_{1} / M_{3}-c_{2} / M_{3}\right)}- \\
& \frac{c_{2} / M_{2}}{\left(c_{1} / M_{1}+c_{2} / M_{2}+1 / M_{3}-c_{1} / M_{3}-c_{2} / M_{3}\right)^{2}} \frac{d c_{1}}{d x} \frac{1}{M_{1}}- \\
& \frac{c_{2} / M_{2}}{\left(c_{1} / M_{1}+c_{2} / M_{2}+1 / M_{3}-c_{1} / M_{3}-c_{2} / M_{3}\right)^{2}} \frac{d c_{2}}{d x} \frac{1}{M_{2}}+ \\
& \frac{c_{2} / M_{2}}{\left(c_{1} / M_{1}+c_{2} / M_{2}+1 / M_{3}-c_{1} / M_{3}-c_{2} / M_{3}\right)^{2}} \frac{d c_{1}}{d x} \frac{1}{M_{3}}+ \\
& \mathrm{c}_{2} \\
& \frac{c_{2} / M_{2}}{\left(c_{1} / M_{1}+c_{2} / M_{2}+1 / M_{3}-c_{1} / M_{3}-c_{2} / M_{3}\right)^{2}} \frac{d c_{2}}{d x} \frac{1}{M_{3}}
\end{aligned}
$$

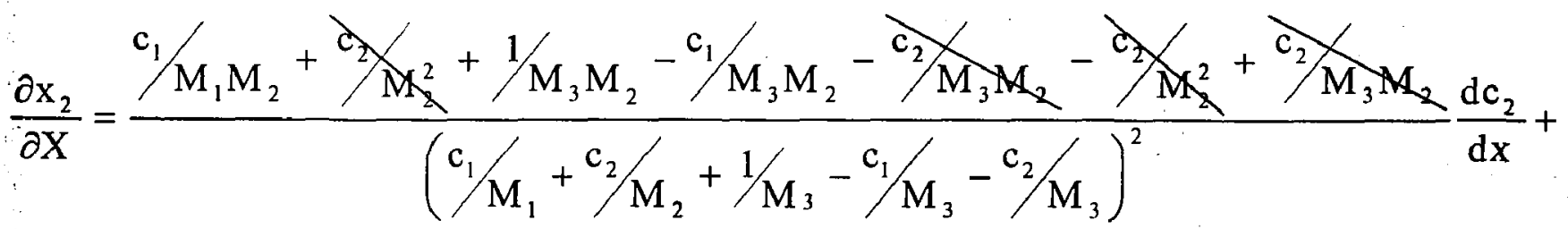

$$
\begin{aligned}
& \frac{-c_{2} / M_{1} M_{2}+c_{2} / M_{1} M_{3}}{\left(c_{1} / M_{1}+c_{2} / M_{2}+1 / M_{3}-c_{1} / M_{3}-c_{2} / M_{3}\right)^{2}} \frac{d c_{1}}{d x}
\end{aligned}
$$




$$
\begin{aligned}
& \frac{\partial x_{2}}{\partial X}=\frac{c_{1} / M_{2} M_{1}+1 / M_{2} M_{3}-c_{1} / M_{3} M_{2}}{\left(c_{1} / M_{1}+c_{2} / M_{2}+1 / M_{3}-c_{1} / M_{3}-c_{2} / M_{3}\right)^{2}} \frac{d c_{2}}{d x}+ \\
& \frac{-c_{2} / M_{2} M_{1}+c_{2} / M_{2} M_{3}}{\left.c_{2} / M_{2}+1 / M_{3}-c_{1} / M_{3}-c_{2} / M_{3}\right)^{2}} \frac{d c_{1}}{d x}
\end{aligned}
$$

$$
\begin{gathered}
\frac{\partial x_{2}}{\partial X}=\frac{\frac{M_{1}+c_{1} M_{3}-c_{1} M_{1}}{M_{1} M_{2} M_{3}}}{\left(\frac{\left.c_{1} / M_{1}+c_{2} / M_{2}+1 / M_{3}-c_{1} / M_{3}-c_{2} / M_{3}\right)^{2}}{d x}\right.} \frac{d c_{2}}{\frac{-c_{2} M_{3}+c_{2} M_{1}}{M_{1} M_{2} M_{3}}}+ \\
\frac{\left.d c_{1} / M_{1}+c_{2} / M_{2}+1 / M_{3}-c_{1} / M_{3}-c_{2} / M_{3}\right)^{2}}{d x}
\end{gathered}
$$

$$
\nabla x_{2}=\frac{M^{2}}{M_{1} M_{2} M_{3}}\left(M_{1}+c_{1} M_{3}-c_{1} M_{1}\right) \nabla c_{2}+\frac{M^{2}}{M_{1} M_{2} M_{3}}\left(c_{2} M_{1}-c_{2} M_{3}\right) \nabla c_{1}
$$


Relating mass flux to the molar flux for component $A$

$$
\begin{aligned}
& j_{A}^{*}=j_{A}-\rho_{A}\left(\frac{x_{A}}{\rho_{A}} j_{A}+\frac{x_{B}}{\rho_{B}} j_{B}+\frac{x_{C}}{\rho_{C}} j_{C}\right) \\
& j_{A}^{*}=\left(1-x_{A}\right) j_{A}-\frac{\rho_{A}}{\rho_{B}} x_{B} j_{B}-\frac{\rho_{A}}{\rho_{C}} x_{C} j_{C}
\end{aligned}
$$

Since

$$
\begin{aligned}
& \frac{1}{\rho}\left(j_{A}+j_{B}+j_{C}\right)=0 \\
& \therefore j_{C}=-j_{A}-j_{B} \\
& j_{A}^{*}=\left(1-x_{A}\right) j_{A}-\frac{\rho_{A}}{\rho_{B}} x_{B} j_{B}-\frac{\rho_{A}}{\rho_{C}} x_{C}\left[-j_{A}-j_{B}\right] \\
& j_{A}=\left(1-x_{A}\right) j_{A}-\frac{\rho_{A}}{\rho_{B}} x_{B} j_{B}+\frac{\rho_{A}}{\rho_{C}} x_{C} j_{A}+\frac{\rho_{A}}{\rho_{C}} x_{C} j_{B} \\
& j_{A}^{*}=\left[\left(1-x_{A}\right)+\frac{\rho_{A}}{\rho_{C}} x_{C}\right] j_{A}+\left[\frac{\rho_{A}}{\rho_{C}} x_{C}-\frac{\rho_{A}}{\rho_{B}} x_{B}\right] j_{B}
\end{aligned}
$$

Relating mass flux to the molar flux for component B

$$
\begin{aligned}
& j_{B}^{*}=j_{B}-\rho_{B}\left(\frac{x_{A}}{\rho_{A}} j_{A}+\frac{x_{B}}{\rho_{B}} j_{B}+\frac{x_{C}}{\rho_{C}} j_{C}\right) \\
& \dot{j}_{B}=\left(1-x_{B}\right) j_{A}-\frac{\rho_{B}}{\rho_{A}} x_{A} j_{A}-\frac{\rho_{B}}{\rho_{C}} x_{C} j_{C}
\end{aligned}
$$


Since

$$
\begin{aligned}
& \frac{1}{\rho}\left(j_{A}+j_{B}+j_{C}\right)=0 \\
& \therefore j_{C}=-j_{A}-j_{B} \\
& j_{B}^{*}=\left(1-x_{B}\right) j_{B}-\frac{\rho_{B}}{\rho_{A}} x_{A} j_{A}-\frac{\rho_{B}}{\rho_{C}} x_{C}\left[-j_{A}-j_{B}\right] \\
& j_{B}^{*}=\left(1-x_{B}\right) j_{B}-\frac{\rho_{B}}{\rho_{A}} x_{A} j_{A}+\frac{\rho_{B}}{\rho_{C}} x_{C} j_{A}+\frac{\rho_{B}}{\rho_{C}} x_{C} j_{B} \\
& j_{B}^{*}=\left[\frac{\rho_{B}}{\rho_{C}} x_{C}-\frac{\rho_{B}}{\rho_{A}} x_{A}\right] j_{A}+\left[\left(1-x_{B}\right)+\frac{\rho_{B}}{\rho_{C}} x_{C}\right] j_{B}
\end{aligned}
$$

\section{Relating mass averaged mass flux to the mole averaged molar flux}

$$
\left[\begin{array}{c}
J_{A}^{*} \\
J_{B}^{*}
\end{array}\right]=\left[\begin{array}{cc}
\frac{1}{M_{A}} & 0 \\
0 & \frac{1}{M_{B}}
\end{array}\right]\left[\begin{array}{c}
j_{A}^{*} \\
j_{B}^{*}
\end{array}\right]
$$

where;

$$
\left[\begin{array}{c}
j_{A}^{*} \\
j_{B}^{*}
\end{array}\right]=\left[\begin{array}{cc}
\left(1-x_{A}\right)+\frac{\rho_{A}}{\rho_{C}} x_{C} & \frac{\rho_{A}}{\rho_{C}} x_{C}-\frac{\rho_{A}}{\rho_{B}} x_{B} \\
\frac{\rho_{B}}{\rho_{C}} x_{C}-\frac{\rho_{B}}{\rho_{A}} x_{A} & \left(1-x_{B}\right)+\frac{\rho_{B}}{\rho_{C}} x_{C}
\end{array}\right]\left[\begin{array}{l}
j_{A} \\
j_{B}
\end{array}\right]
$$

Therefore,

$$
\left[\begin{array}{l}
J_{A}^{*} \\
J_{B}^{*}
\end{array}\right]=\left[\begin{array}{cc}
\frac{1}{M_{A}}\left[\left(1-x_{A}\right)+\frac{\rho_{A}}{\rho_{C}} x_{C}\right] & \frac{1}{M_{A}}\left[\frac{\rho_{A}}{\rho_{C}} x_{c}-\frac{\rho_{A}}{\rho_{B}} x_{B}\right] \\
\frac{1}{M_{B}}\left[\frac{\rho_{B}}{\rho_{C}} x_{c}-\frac{\rho_{B}}{\rho_{A}} x_{A}\right] & \frac{1}{M_{B}}\left[\left(1-x_{B}\right)+\frac{\rho_{B}}{\rho_{C}} x_{C}\right]
\end{array}\right]\left[\begin{array}{l}
j_{A} \\
j_{B}
\end{array}\right]
$$


APPENDIX B 


\section{APPENDIX B \\ FORTRAN program}

Based on the Firoozabadi model, a computer code was written in FORTRAN for a twodimensional domain. In this software package, there are eleven source files and three data input files. Their functions are introduced here.

\section{Main.f}

This is the main control program. It sets up all the variables and data blocks, and calls all other subroutines to conduct the calculation.

Init.f

This is the subroutine to set the initial condition for a new run based on previous results.

Mail.f

This is the subroutine is used to coordinate calculation based on a given discretization scheme for a given rectangular domain.

Coefficients.f

This is the subroutine to calculate all three diffusion coefficients, based on given temperature, pressure, and compositions in a mixture.

Pressure.f

This is a subroutine to solve the pressure equation. 


\section{Velocity.f}

This subroutine is used to calculate velocity based on Darcy's Law and available pressure gradients.

\section{Energy.f}

This subroutine solves the energy equation.

\section{Concentration.f}

This subroutine solves the concentration equation.

\section{Stream.f}

This subroutine solves the streamline function equation.

\section{Linear_Solver.f}

This subroutine is used to adjust data for any linear system, and call the bi-conjugate gradient method to solve it.

nspcg.f

This subroutine is used to implement the bi-conjugate gradient method.

\section{Data.in}

This is a data file that provides all control data. 
Grid_size.in

This data file defines the two dimensional domain and perform discretization of the domain.

Pure_comp_prop.in

This is a data file that provides all necessary properties for each of the pure components in the mixture.

The structure of the program is shown in Figure 1-B.

During the course of this research, the data transfer between each routine was improved, expanded for a ternary mixture, simplified, and any unnecessary statements were removed. The new version of the program was tested on the same problem and verified by comparing the predictions with the original results. 


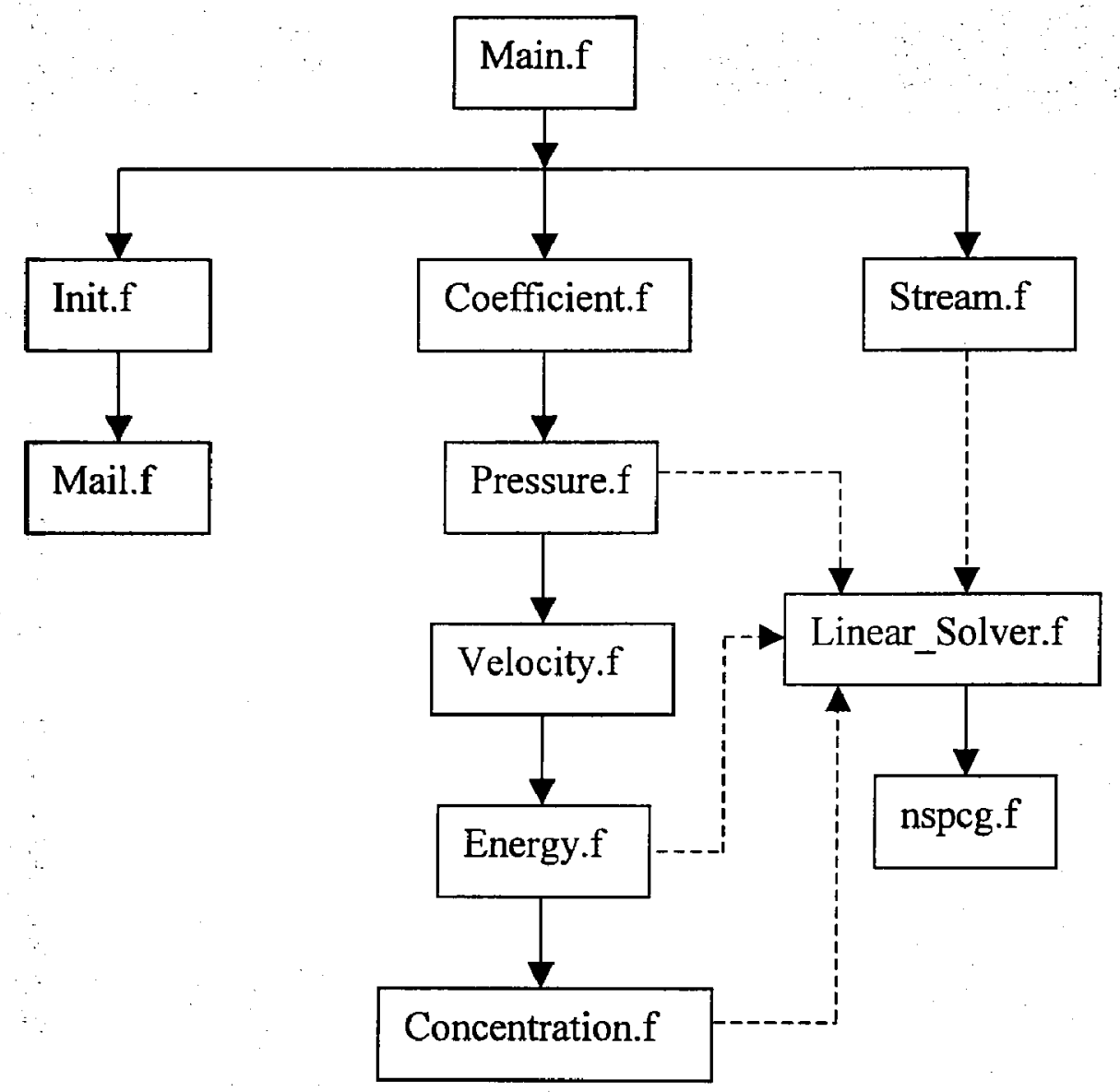

Figure 1-B: Flow chart of the FORTRAN program

$3 \cdot 94 \cdot 50$ 